\title{
A Theory of $L^{1}$-Dissipative Solvers for Scalar Conservation Laws with Discontinuous Flux
}

\author{
Boris Andreianov, Kenneth Hvistendahl Karlsen \& Nils \\ HENRIK RISEBRO
}

Communicated by C. DAFERMOS

\begin{abstract}
We propose a general framework for the study of $L^{1}$ contractive semigroups of solutions to conservation laws with discontinuous flux:

$$
u_{t}+\mathfrak{f}(x, u)_{x}=0, \quad \mathfrak{f}(x, u)= \begin{cases}f^{l}(u), & x<0, \\ f^{r}(u), & x>0,\end{cases}
$$

where the fluxes $f^{l}, f^{r}$ are mainly assumed to be continuous. Developing the ideas of a number of preceding works (BAITI and JENSSEN in J Differ Equ 140(1):161185, 1997; Towers in SIAM J Numer Anal 38(2):681-698, 2000; Towers in SIAM J Numer Anal 39(4):1197-1218, 2001; Towers et al. in Skr K Nor Vidensk Selsk 3:1-49, 2003; Adimurthi ET AL. in J Math Kyoto University 43(1):27-70, 2003; Adimurthi et Al. in J Hyperbolic Differ Equ 2(4):783-837, 2005; Audusse and Perthame in Proc Roy Soc Edinburgh A 135(2):253-265, 2005; Garavello ET AL. in Netw Heterog Media 2:159-179, 2007; BÜRGER ET AL. in SIAM J Numer Anal 47:1684-1712, 2009), we claim that the whole admissibility issue is reduced to the selection of a family of "elementary solutions", which are piecewise constant weak solutions of the form

$$
c(x)=c^{l} 11_{\{x<0\}}+c^{r} 11_{\{x>0\}} .
$$

We refer to such a family as a "germ". It is well known that (CL) admits many different $L^{1}$ contractive semigroups, some of which reflect different physical applications. We revisit a number of the existing admissibility (or entropy) conditions and identify the germs that underly these conditions. We devote specific attention to the "vanishing viscosity" germ, which is a way of expressing the " $\Gamma$-condition" of DieHL (J Hyperbolic Differ Equ 6(1):127-159, 2009). For any given germ, we formulate "germ-based" admissibility conditions in the form of a trace condition on the flux discontinuity line $\{x=0\}$ [in the spirit of VOL'PERT (Math USSR Sbornik 2(2):225-267, 1967)] and in the form of a family of global
\end{abstract}


entropy inequalities [following KRUZHKOV (Math USSR Sbornik 10(2):217-243, 1970) and CARrillo (Arch Ration Mech Anal 147(4):269-361, 1999)]. We characterize those germs that lead to the $L^{1}$-contraction property for the associated admissible solutions. Our approach offers a streamlined and unifying perspective on many of the known entropy conditions, making it possible to recover earlier uniqueness results under weaker conditions than before, and to provide new results for other less studied problems. Several strategies for proving the existence of admissible solutions are discussed, and existence results are given for fluxes satisfying some additional conditions. These are based on convergence results either for the vanishing viscosity method (with standard viscosity or with specific viscosities "adapted" to the choice of a germ), or for specific germ-adapted finite volume schemes.

\section{Contents}

1. Introduction . . . . . . . . . . . . . . . . . . . . . . . . . 29

1.1. $L^{1}$-dissipativity for scalar conservation laws . . . . . . . . . . . . . . . 29

1.2. Analysis in terms of Riemann solvers . . . . . . . . . . . . . . . . . . . 31

1.3. Admissibility germs and uniqueness . . . . . . . . . . . . . . 32

1.4. Measure-valued $\mathcal{G}$-entropy solutions and convergence results . . . . . . . . . . 34

1.5. Outline of the paper . . . . . . . . . . . . . . . . . . . . 34

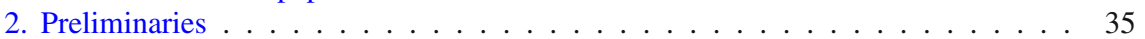

3. The model one-dimensional problem . . . . . . . . . . . . . . . . . 37

3.1. Definitions of germs and their basic properties . . . . . . . . . . . . . . 37

3.2. Definitions and uniqueness of $\mathcal{G}$-entropy solutions . . . . . . . . . . . . . . 39

3.3. Comparison and continuous dependence results and $L^{\infty}$ estimates . . . . . . 45

3.4. Complete germs and $\mathcal{G}$-entropy process solutions . . . . . . . . . . . . 47

3.5. Conclusions . . . . . . . . . . . . . . . . . . 50

4. Examples and analysis of known admissibility criteria . . . . . . . . . . . . . . 51

4.1. More about complete germs and closed germs . . . . . . . . . . . . . . . . 51

4.2. The case $f^{l} \equiv f^{r}$; the Vol'pert-Kruzhkov germ . . . . . . . . . . . . . . . . 54

4.3. The case $f^{l} \equiv f^{r}$; the Gelfand germ . . . . . . . . . . . . . . 55

4.4. Increasing surjective fluxes $f^{l, r} \ldots \ldots \ldots \ldots \ldots$

4.5. Other cases with monotone fluxes $f^{l, r} \ldots \ldots \ldots$. . . . . . . . . . . . . . . 57

4.6. The Audusse-Perthame adapted entropies . . . . . . . . . . . . . . . 57

4.7. The Karlsen-Risebro-Towers entropy condition . . . . . . . . . . . . . . . 60

4.8. Bell-shaped fluxes . . . . . . . . . . . . . . . . . . . 63

4.9. The case $f^{l} \equiv f^{r}$ and the Colombo-Goatin germs . . . . . . . . . . . 64

5. The vanishing viscosity germ . . . . . . . . . . . . . . . . 65

6. Some existence and convergence results . . . . . . . . . . . . . . 70

6.1. The standard vanishing viscosity approach . . . . . . . . . . . . 71

6.2. The vanishing viscosity approach adapted to $(A, B)$-connections . . . . . . 74

6.3. Existence for complete germs through the discretization approach . . . . . . 76

6.4. On convergence of approximate solutions without $B V$ estimates $\ldots . . . . \quad 80$

Acknowledgements . . . . . . . . . . . . . . . . . . . 81

References . . . . . . . . . . . . . . . . . . . . . 82 


\section{Introduction}

\section{1. $L^{1}$-dissipativity for scalar conservation laws}

We are interested in the well-posedness of the Cauchy problem for a general scalar conservation law:

$$
\begin{gathered}
u_{t}+\operatorname{div} \mathfrak{f}(t, x, u)=0, \\
\left.u\right|_{t=0}=u_{0} .
\end{gathered}
$$

It is well known that classical solutions to this problem may not exist globally since discontinuities can develop in finite time; hence (1.1),(1.2) must be interpreted in the weak (distributional) sense. However, weak solutions are, in general, not unique, and so additional admissibility criteria are needed to single out a unique solution; these so-called entropy conditions are usually motivated by a very careful inspection of the underlying physical phenomena in presence of discontinuous or rapidly changing solutions.

Consider solutions which take values in a closed interval $U \subset \mathbb{R}$. Whenever the flux function $\mathfrak{f}: \mathbb{R}_{+} \times \mathbb{R}^{N} \times U \mapsto \mathbb{R}^{N}$ is sufficiently regular in all variables, the classical notion of Kruzhkov entropy solutions in the $L^{\infty}$ framework [54] (see VOL'PERT [84] for the $B V$ setting and OLENIK [65]) and its further extensions to bounded domains, measure-valued solutions, renormalized solutions, and degenerate parabolic problems, see, for example [7,16,18,26,61,67,70,72], have provided a rather complete theory of well-posedness. An equivalent notion of kinetic solutions was later formulated in $[60,75]$, which allowed for a deeper study of regularity and compactness properties of admissible solutions. By far the most studied case is the autonomous equation

$$
u_{t}+\operatorname{div} \mathfrak{f}(u)=0
$$

Under mild regularity assumptions on the flux $\mathfrak{f}$, it is well known that the Kruzhkov entropy solutions of (1.2),(1.3) (with $u_{0} \in L^{1} \cap L^{\infty}$ ) form an $L^{1}$-contractive and order-preserving semigroup on $L^{1} \cap L^{\infty}[8,17,19,32,54-56,62,76]$. Moreover, this is the unique semigroup of this kind which admits all the trivial constant solutions of (1.3). This semigroup is generally viewed as the one representing the physically relevant solutions to scalar conservation laws. Solution semigroups that are Lipschitz in $L^{1}$ but not contractive may exist and are of physical interest (see [58]), but their study will not be addressed in the present paper.

A comparable theory for conservation laws with discontinuous flux and related degenerate parabolic equations is still not available, although these equations have received intense attention in last 15 years; see [1-4,13-15,20-25,28,31,34-37, 40,42,43,46-53,63, 64, 66, 73, 74,77, 80,81] (and additional references therein) for a number of different admissibility criteria, existence and/or uniqueness results, which we partially revisit in Section 4. Recently, it was pointed out explicitly by Adimurthi et AL. [4] that for the case $\mathfrak{f}=\mathfrak{f}(x, u)$ with $\mathfrak{f}$ piecewise constant in $x$, there may exist many different $L^{1}$-contractive semigroups of solutions to (1.1). Different semigroups correspond to different physical phenomena modeled by the 
same equation, but with different dissipative processes occurring on the discontinuities of the flux. We refer to [21] for a comparison of a clarifier-thickener model and a porous medium model, which leads to the same formal conservation law, but with two distinct semigroups of physically relevant solutions. Notice that even for the classical equation (1.3), non-Kruzhkov $L^{1}$-contractive semigroups can also be constructed (see $[9,31]$ ), by an approach much similar to the one of $[4,22]$ and the present paper.

In this paper we formulate a streamlined, unifying framework encompassing the different notions of entropy solutions for (1.1). For the multi-dimensional problem examined in the sequel paper [10] we consider Carathéodory functions $\mathfrak{f}(t, x, u)$ that are piecewise Lipschitz continuous in $(t, x)$ and locally Lipschitz continuous in $u$. The present paper focuses on the model one-dimensional case with

$$
\mathfrak{f}=\mathfrak{f}(x, u)=f^{l}(u) 1_{\{x<0\}}+f^{r}(u) 1_{\{x>0\}},
$$

and $f^{l}, f^{r}: U \rightarrow \mathbb{R}$ being merely continuous (in the existing literature the fluxes are usually assumed to be Lipschitz continuous). We note that a generalization to a piecewise constant in $x$ and continuous in $u$ flux function $\mathfrak{f}(x, u)$ is straightforward.

The initial datum $u_{0}$ is assumed to belong to $L^{\infty}(\mathbb{R} ; U)$. The presence of a source term $s(t, x)$ in the conservation law (1.1) is easy to take into account, for example in the case $U=\mathbb{R}$ and $s(t, \cdot) \in L^{\infty}\left(\mathbb{R}^{N}\right)$ for almost every $t>0$ with $\int_{0}^{T}\|s(t, \cdot)\|_{L^{\infty}} \mathrm{d} t<\infty$ for all $T>0$. For the sake of simplicity, we always take $s \equiv 0$.

Definition 1.1. A semigroup $\left(S_{t}\right)_{t \geqq 0}, S_{t}: D \rightarrow L^{\infty}(\mathbb{R} ; U)$, defined on a subset $D \subset L^{\infty}(\mathbb{R} ; U)$, is called an $L^{1}$-dissipative solver for (1.1),(1.4) if

- for all $u_{0} \in D$, the trajectory $u(t, x):=S_{t}\left(u_{0}\right)(x)$ of the semigroup $S_{t}$ gives a solution $^{1}$ to the problem (1.1) with flux (1.4) and initial data $u_{0}$.

- for all $u_{0}, \hat{u}_{0} \in D, u:=S_{t}\left(u_{0}\right)$ and $\hat{u}:=S_{t}\left(\hat{u}_{0}\right)$ satisfy the "Kato inequality"

$$
\begin{aligned}
& \forall \xi \in \mathcal{D}([0, \infty) \times \mathbb{R}), \quad \xi \geqq 0, \\
& -\int_{\mathbb{R}_{+}} \int_{\mathbb{R}}\left\{|u-\hat{u}| \xi_{t}+\operatorname{sign}(u-\hat{u})(\mathfrak{f}(x, u)-\mathfrak{f}(x, \hat{u})) \xi_{x}\right\} \\
& \quad \leqq \int_{\mathbb{R}}\left|u_{0}-\hat{u}_{0}\right| \xi(0, x) .
\end{aligned}
$$

Letting $\xi$ go to the characteristic function of $(0, t) \times \mathbb{R}$ (see, for example $[17$, $55])$, we deduce from (1.5) the $L^{1}$-contractivity property

$$
\begin{aligned}
& \text { whenever }\left|u_{0}-\hat{u}_{0}\right| \in L^{1}(\mathbb{R}), \quad \int_{\mathbb{R}}|u-\hat{u}|(t) \leqq \int_{\mathbb{R}}\left|u_{0}-\hat{u}_{0}\right| \\
& \text { for almost every } t>0 .
\end{aligned}
$$

We could also have required an $L^{1}$-dissipative solver to be order-preserving, in which case $\operatorname{sign}(u-\hat{u})$ and $|u-\hat{u}|$ in (1.5),(1.6) are replaced by $\operatorname{sign}^{+}(u-\hat{u})$ and

1 That is, a weak solution which is admissible in a sense specified later. 
$(u-\hat{u})^{+}$, respectively. It turns out that the $L^{1}$-dissipative solvers that we consider are automatically order-preserving (see [33]).

Notice that in several spatial dimensions mere continuity of the flux $\mathfrak{f}$ in $u$ is not sufficient to obtain $L^{1}$ contractivity from the Kato inequality; see in particular the counterexample of KRUZhKov and PANOv [56]; see [8, 17, 19,55,62] for some sufficient conditions for (1.6) to hold.

\subsection{Analysis in terms of Riemann solvers}

Our primary goal is to formulate a convenient unifying framework for the study of different $L^{1}$-dissipative solvers. We will work with the basic one-dimensional model equation

$$
u_{t}+\mathfrak{f}(x, u)_{x}=0, \quad \mathfrak{f}=\mathfrak{f}(x, u)=f^{l}(u) \mathbb{1}_{\{x<0\}}+f^{r}(u) \mathbb{1}_{\{x>0\}},
$$

which the existing literature has targeted as the main example for understanding the admissibility issue in the case of a spatially discontinuous flux.

With the admissibility of solutions in the regions $\{x>0\}$ and $\{x<0\}$ being understood in the sense of Kruzhkov's entropy solutions, it remains to define the admissibility of a solution at the discontinuity set $\Sigma=(0, \infty) \times\{0\}$. As suggested by GARAVELlo ET AL. [40], the admissibility issue should reduce to the choice of a Riemann solver at $x=0$. That is, to each pair $\left(u_{-}, u_{+}\right) \in U \times U$ we have to assign a weak solution $u:=\mathcal{R} \mathcal{S}\left(u_{-}, u_{+}\right)$of (1.7) with the initial datum

$$
u_{0}(x)= \begin{cases}u_{-}, & x<0 \\ u_{+}, & x>0\end{cases}
$$

If (1.5) and (1.6) turn out to be true, such a solution should be unique. If we assume that

$$
\begin{aligned}
& \text { the notion of solution for (1.7) is invariant } \\
& \text { under the scaling }(t, x) \mapsto(k t, k x), k>0 \text {, }
\end{aligned}
$$

it follows that the unique solution to (1.7), (1.8) is self-similar, that is, it depends only on the ratio $\xi:=x / t$. Furthermore, (1.5) should hold with $u, \hat{u}$ being solutions $\mathcal{R S}\left(u_{-}, u_{+}\right), \mathcal{R S}\left(\hat{u}_{-}, \hat{u}_{+}\right)$of two different Riemann problems.

If, in addition, we assume

any solution of (1.7) is a Kruzhkov entropy solution in $\mathbb{R}_{+} \times(\mathbb{R} \backslash\{0\})$,

it follows that the solution $u=\mathcal{R S}\left(u_{-}, u_{+}\right)$is monotone in $\xi$ on each side of the discontinuity; thus, it has strong pointwise left and right traces on $\Sigma=(0, \infty) \times\{0\}$. Let us denote these traces by $\gamma^{l} u$ and $\gamma^{r} u$, respectively. If $u^{l, r}:=\gamma^{l, r} u$, the weak formulation of (1.7) yields the Rankine-Hugoniot condition

$$
f^{l}\left(u^{l}\right)=f^{r}\left(u^{r}\right) .
$$


Hence, we can associate to the pair $\left(u^{l}, u^{r}\right)$ the following stationary weak solution of (1.7):

$$
\begin{cases}u^{l}, & x<0, \\ u^{r}, & x>0 .\end{cases}
$$

Any solution of the form (1.10), with $\left(u^{l}, u^{r}\right) \in U \times U$, will be called an "elementary solution", and be identified with the pair $\left(u^{l}, u^{r}\right)$.

Under assumptions (A1) and (A2), we conclude that the solution $\mathcal{R} \mathcal{S}\left(u_{-}, u_{+}\right)$ of the Riemann problem should consist of the following ingredients:

- $\quad$ the standard Kruzhkov self-similar solution joining the state $u_{-}$ at $t=0, x<0$ to some state $u^{l}$ at $t>0, x=0^{-}$;

(RPb-sol.) - the jump joining the state $u^{l}$ at $x=0^{-}$to a state $u^{r}$ at $x=0^{+}$
such that (1.9) holds;

- $\quad$ the standard Kruzhkov self-similar solution joining the state $u^{r}$ at $t>0, x=0^{+}$to the state $u_{+}$at $t=0, x>0$.

Remark 1.2. In (RPb-sol.), it is convenient to consider the situation in which the wave fans joining $u_{-}$to $u^{l}$, respectively $u^{r}$ to $u_{+}$, might contain zero-speed shocks. In this case, the same almost everywhere defined solution may correspond to two or more different pairs $\left(u^{l}, u^{r}\right)$ of intermediate states at $x=0^{ \pm}$, and $\gamma^{l, r} u$ may differ from $u^{l, r}$.

Whenever solutions of Riemann problems are seen as trajectories of an $L^{1}$-dissipative solver for (1.7), the different "elementary solution" pairs $\left(u^{l}, u^{r}\right),\left(\hat{u}^{l}, \hat{u}^{r}\right)$ that can be used in ( $\mathrm{RPb}$-sol) ought to satisfy

$$
\begin{aligned}
q^{l}\left(u^{l}, \hat{u}^{l}\right) & :=\operatorname{sign}\left(u^{l}-\hat{u}^{l}\right)\left(f^{l}\left(u^{l}\right)-f^{l}\left(\hat{u}^{l}\right)\right) \\
& \geqq \operatorname{sign}\left(u^{r}-\hat{u}^{r}\right)\left(f^{r}\left(u^{r}\right)-f^{r}\left(\hat{u}^{r}\right)\right)=: q^{r}\left(u^{r}, \hat{u}^{r}\right) .
\end{aligned}
$$

Indeed, denote by $\hat{u}^{l, r}$ the left and right traces on $\Sigma$ of $\hat{u}=\mathcal{R} \mathcal{S}\left(\hat{u}_{-}, \hat{u}_{+}\right)$and let the test function $\xi$ in the Kato inequality (1.5) converge to the characteristic function of $(0, T) \times\{0\}$. We then easily arrive at (1.11).

\subsection{Admissibility germs and uniqueness}

The property in (1.11) of allowed jumps across $\Sigma$ has been recognized in many works as crucial for the uniqueness of admissible solutions; it was the key ingredient of the uniqueness proofs in, for example, the works $[4,22,40]$.

Our angle of attack is to "axiomatize" property (1.11). More precisely, we claim that admissibility can be defined directly from the choice of a set $\mathcal{G} \subset U \times U \subset \mathbb{R}^{2}$ such that (1.9) and (1.11) hold for all $\left(u^{l}, u^{r}\right),\left(\hat{u}^{l}, \hat{u}^{r}\right) \in \mathcal{G}$. Such a set $\mathcal{G}$ will be called an $L^{1}$-dissipative admissibility germ (an $L^{1} D$ germ for short). If an $L^{1} D$ germ $\mathcal{G}$ admits a unique maximal extension, still satisfying (1.9) and (1.11), the germ is said to be definite.

Our results in Section 3 can be formulated as follows: if $\mathcal{G}$ is a definite germ, it corresponds to a unique $L^{1}$-dissipative solver $S_{t}^{\mathcal{G}}$ for (1.7) such that 
- for any trajectory $u$ of $S_{t}^{\mathcal{G}}$, the restrictions of $u$ to the domains $\mathbb{R}_{+} \times(-\infty, 0)$ and $\mathbb{R}_{+} \times(0, \infty)$ are entropy solutions

$\left(S_{t}^{\mathcal{G}}\right.$-sol. $) \quad$ in the sense of Kruzhkov;

- roughly speaking, the pair of left and right traces $\left(\gamma^{l} u, \gamma^{r} u\right)$ of $u$ on $\mathbb{R}_{+} \times\{0\}$ belongs to $\mathcal{G}^{*}$ pointwise, where $\mathcal{G}^{*}$ is the unique maximal $L^{1} D$ extension of $\mathcal{G}$.

The set $\mathcal{G}^{*}$ is exactly the set of "elementary solutions" contained within $S_{t}^{\mathcal{G}}$. Without non-degeneracy assumptions on the fluxes $f^{l}$ and $f^{r}$, the second statement in $\left(S_{t}^{\mathcal{G}}\right.$-sol.) should be made precise. Following the idea of $[78,82]$, the pair of (weak) traces $\left(\gamma^{l} u, \gamma^{r} u\right)$ can be defined as a Young measure on $\mathbb{R}^{2}$, which is the "nonlinear weak- $\star$ limit" (see $[38,39])$ of $\left(u\left(t,-h_{n}\right), u\left(t, h_{n}\right)\right)$, with $\left(h_{n}\right)_{n>1}, h_{n} \downarrow 0$, being a sequence of Lebesgue points of the map $x \mapsto(u(\cdot,-x), u(\cdot, x))$. What we mean is that for almost every $t>0$, the support of this Young measure is contained in a part of $\mathcal{G}^{*}$ [a part on which, moreover, the function $\left(u^{l}, u^{r}\right) \mapsto\left(f^{l}\left(u^{l}\right), f^{r}\left(u^{r}\right)\right)$ is constant, see Section 3]. A more practical way to understand the boundary trace issue is suggested in Definition 3.8(ii) in Section 3.

Proposition 3.10 (i) in Section 3 shows that the elementary solutions on which the admissibility criterion is based actually belong to $S_{t}^{\mathcal{G}}$, that is, they are admissible. ${ }^{2}$ From this viewpoint, our approach generalizes the original one of Kruzhkov by defining admissibility in terms of the local $L^{1}$-dissipativity property (1.5) with respect to a "small" set of solutions that are judged admissible a priori. ${ }^{3}$ In the Kruzhkov case, these are the constant solutions of $u_{t}+f(u)_{x}=0$ (see Section 4.2). In our context, these are the elementary solutions of the form (1.10) with $\left(u^{l}, u^{r}\right) \in \mathcal{G}$, and the constant solutions in the domains $\mathbb{R}_{+} \times(-\infty, 0)$ and $\mathbb{R}_{+} \times(0, \infty)$.

Our perspective has the following practical implications:

First, there exist in the literature a number of different admissibility criteria devised for particular forms of the fluxes $f^{l}, f^{r}$. For some of them, the uniqueness and/or existence issues were left open; and it is often difficult to judge whether the same criteria can be useful for fluxes more general than those initially considered. On the other hand, if we manage to find some of the elementary solutions allowed by a given admissibility criterion in a given configuration of fluxes $f^{l}, f^{r}$, it becomes possible to determine if the criterion is adequate. We give examples in Section 4.

Second, some important admissibility criteria derive from certain regularized problems. For example, for the classical vanishing viscosity method, which consists in adding $\varepsilon \Delta u$ to the right-hand side of (1.1) (see HopF [45] and KRUZHKOV [54]), a variant of the Kato inequality (1.5) is well known for each $\varepsilon>0$. Hence,

${ }^{2}$ Notice that if $\mathcal{G}$ is not $L^{1} D$, then the admissibility of some of its elementary solutions is contradictory: the pairs $\left(u^{l}, u^{r}\right),\left(\hat{u}^{l}, \hat{u}^{r}\right)$ for which (1.11) fails rule out each other!

3 A well-known alternative (see BÉNILAN [17] and CRANDALl [32]) is to define admissibility by the global $L^{1}$-contraction property (1.6) with respect to a dense set of solutions of the stationary problem $u+f(u)_{x}=s$; this is the nonlinear semigroup approach to scalar conservation laws. We will not develop this second possibility here. From another point of view, however, the definition of admissibility on the flux discontinuity line $\{x=0\}$ by property (1.11) is a direct generalization of the admissibility approach of VOL'PERT [84]; see Section 4.2. 
the Kato inequality is easily passed on to the limiting problem, which in turn gives rise to an implicitly defined $L^{1}$-dissipative solver for the limit problem. Determining the elementary solutions (as $\varepsilon \rightarrow 0$ limits of standing-wave profiles, for example) opens up for the possibility of characterizing the germ of the associated $L^{1}$-dissipative solver, and as a consequence describe it explicitly. This can eventually lead to an existence result. Examples are given in Sections 5 and 6. Our analysis utilizes several simple properties of germs listed in Sections 3 and 4.

Finally, we point out that while the formulation $\left(S_{t}^{\mathcal{G}}\right.$-sol.) (see Definition 3.8) is convenient for the uniqueness proof, it is rather ill-suited for passing to the limit in a sequence of approximate solutions. Therefore, we supply another formulation in terms of global entropy inequalities, inspired by the previous works of BAITI and Jenssen [15], Audusse and Perthame [13], Bürger et Al. [22], and by the founding papers of KruzhKov [54], OTTo [67], and CARrillo [26]. This formulation (see Definition 3.15 for the precise notion) is also based upon a predefined $L^{1} D$ germ $\mathcal{G}$ associated with the flux discontinuity. Stability of this formulation with respect to $L_{\text {loc }}^{1}$ convergence of sequences of (approximate) solutions makes it convenient for the existence analysis. It is shown in Theorem 3.18 that the two formulations are equivalent. We call the associated solutions $\mathcal{G}$-entropy solutions.

\subsection{Measure-valued $\mathcal{G}$-entropy solutions and convergence results}

The definition of $\mathcal{G}$-entropy solutions in terms of global entropy inequalities can be adapted to define measure-valued $\mathcal{G}$-entropy solutions (more precisely, we use the equivalent device of process solutions developed in $[38,39]$; the same idea appeared previously in [69]). Although we are not able to conceive a direct uniqueness proof for $\mathcal{G}$-entropy process solutions, in Section 3 we prove their uniqueness if we already know the existence of $\mathcal{G}$-entropy solutions. A result like this makes it possible to prove strong convergence of merely bounded sequences of approximate $\mathcal{G}$-entropy solutions. Heuristically, on the condition that existence can be established by some approximation method enjoying strong compactness properties (see Section 6.3), we can deduce strong convergence of other approximation methods with mere $L^{\infty}$ estimates. We give the details in Section 6.4.

\subsection{Outline of the paper}

In Section 2, we recall some parts of the Kruzhkov theory; we also state and slightly reformulate some results by Panov on initial and boundary traces of Kruzhkov entropy solutions. In Section 3, we define admissibility germs and related notions, provide several definitions of $\mathcal{G}$-entropy solutions, discuss the relations between them, provide uniqueness, $L^{1}$-contraction, and comparison results, and $L^{\infty}$ estimates. In Section 4, we derive a series of important properties of germs. Moreover, we classify several known admissibility criteria in terms of their underlying germs, and determine their areas of applicability. An important example is the "vanishing viscosity" admissibility condition. In Section 5, we characterize its germ for general flux functions $f^{l, r}$. In Section 6, we justify the convergence of the standard vanishing viscosity approximations to the "vanishing viscosity germ" 
entropy solutions obtained in Section 5. Furthermore, we discuss variants of the vanishing viscosity approach that lead to different germs; in particular, we give a simplified proof of existence for the " $(A, B)$-connection" admissibility criteria studied by ADIMURTHI ET AL. [4] and by Bürger ET AL. [22]. Then we give an existence result for $\mathcal{G}$-entropy solutions corresponding to a general germ. The proof is a by-product of a strong convergence result for a particular germ-preserving finite volume scheme. Finally, we discuss convergence of $L^{\infty}$ bounded sequences of approximate solutions towards $\mathcal{G}$-entropy process solutions.

\section{Preliminaries}

First, we recall a localized version of the definition due to KRUZHKOV [54]. Let $\Omega \subset \mathbb{R}_{+} \times \mathbb{R}$ be open and set $\partial^{0} \Omega:=\partial \Omega \cap\{t=0\}$. For $f \in C(U ; \mathbb{R})$, a function $u \in L^{\infty}(\Omega ; U)$ satisfying the inequalities

$$
\begin{aligned}
& \forall k \in U \quad \forall \xi \in \mathcal{D}\left(\mathbb{R}_{+} \times \mathbb{R}\right), \quad \xi \geqq 0,\left.\quad \xi\right|_{\partial \Omega \backslash \partial^{0} \Omega}=0, \\
& \iint_{\Omega}\left\{|u-k| \xi_{t}+q(u, k) \xi_{x}\right\}-\iint_{\Omega} \operatorname{sign}(u-k) s \xi \geqq \int_{\partial^{0} \Omega}\left|u_{0}-k\right| \xi,
\end{aligned}
$$

is called a Kruzhkov entropy solution of the conservation law

$$
u_{t}+f(u)_{x}=s \text { in } \Omega,\left.\quad u\right|_{\partial^{0} \Omega}=u_{0} .
$$

Here for $k \in \mathbb{R}$, the function $z \mapsto|z-k|$ is called a Kruzhkov entropy, and

$$
q(z, k):=\operatorname{sign}(u-k)(f(u)-f(k))
$$

is the associated entropy flux.

An important ingredient of our formulation is the fact that entropy solutions admit strong traces, in an appropriate sense. For example, when $\Omega=\mathbb{R}_{+} \times \mathbb{R}$, an entropy solution of (2.1) admits $u_{0}$ as the initial trace in the sense

$$
\forall \xi \in \mathcal{D}(\mathbb{R}) \quad \lim _{h \rightarrow 0^{+}} \frac{1}{h} \iint_{(0, h) \times \mathbb{R}} \xi(x)\left|u(t, x)-u_{0}(x)\right|=0 .
$$

(see PANOv [71] and the previous works of CHEN and RASCLE [30] and of VASSEUR [83]). Thus, $t=0$ can be seen as a Lebesgue point of the map $t \mapsto u(t, \cdot) \in L_{\mathrm{loc}}^{1}(\Omega)$.

Most importantly for the present paper, analogous strong trace results hold for entropy solutions in half-space domains. For example, with $\Omega=\mathbb{R}_{+} \times(-\infty, 0)$, provided the non-degeneracy assumption

$$
\text { for any non-empty interval }(a, b) \subset U,\left.f\right|_{(a, b)} \text { is not constant }
$$

holds, there exists a measurable function $t \mapsto\left(\gamma^{l} u\right)(t)$ on $\mathbb{R}_{+}$such that

$$
\forall \xi \in \mathcal{D}(0, \infty) \quad \lim _{h \rightarrow 0^{+}} \frac{1}{h} \iint_{\mathbb{R}_{+} \times(-h, 0)} \xi(t)\left|u(t, x)-\left(\gamma^{l} u\right)(t)\right|=0 .
$$

This result is a particular case of PANOv [72, Theorem 1.4] (see also [57,83]). Observe that we require from the flux $f$ only that it is continuous and satisfies 
the non-degeneracy condition (2.3). The function $\gamma^{l} u$ is the strong left trace of the entropy solution $u$ on $\{x=0\}$.

We emphasize that (2.4) is a sufficient-but not a necessary-property for our purposes. Actually, strong traces of $f(u)$ and of the entropy fluxes $q(u, k)$ exist even in the absence of the non-degeneracy assumption (2.3). To state the result, we consider a "singular mapping" $V: U \mapsto \mathbb{R}$. There are different choices of the singular mappings; in particular, whenever $f$ is of bounded variation it is convenient to define $V(z)=\int_{0}^{z}|d f(s)|$ (see Temple [79], KLINGEnberg and Risebro [53], and also $[4,14,81])$. To deal with the model problem in hand, let us fix the choice

$$
V(z):=\int_{0}^{z} 1_{E}(s) \mathrm{d} s
$$

where $E$ is the maximal subset of $U$ such that $f(\cdot)$ is non-constant on any nondegenerate interval contained in $E$. Clearly, under the non-degeneracy assumption (2.3), $V$ becomes the identity mapping. Now the result of [72, Theorem 1.4] can be restated in the following way.

Theorem 2.1. Let $\mathfrak{f}: U \rightarrow \mathbb{R}$ be continuous. Assume $u$ is a Kruzhkov entropy solution of $u_{t}+f(u)_{x}=0$ in $\Omega:=\mathbb{R}_{+} \times(-\infty, 0)$. Then $V(u)$ admits a strong left trace on $\{x=0\}$. Namely, there exists a function $t \mapsto\left(\gamma^{l} V(u)\right)(t)$ such that

$$
\forall \xi \in \mathcal{D}(0, \infty), \quad \lim _{h \rightarrow 0^{+}} \frac{1}{h} \iint_{\mathbb{R}_{+} \times(-h, 0)} \xi(t)\left|V(u)(t, x)-\left(\gamma^{l} V(u)\right)(t)\right|=0 .
$$

Similar statements hold with $\Omega=\mathbb{R}_{+} \times(0, \infty)$ and the right trace operator $\gamma^{r}$.

Now let us turn to our problem (1.7). Whenever $u$ is a Kruzhkov entropy solution of (1.7) in the domains $\{x<0\}$ and $\{x>0\}$, by Theorem 2.1 there exist strong one-sided traces $\gamma^{l} V^{l}(u), \gamma^{r} V^{r}(u)$. Here the singular mappings $V^{l, r}$ are defined by (2.5) from $f=f^{l, r}$, respectively.

In the two subsequent remarks, we indicate how to use the strong traces of $V^{l, r}(u)$ to express the strong traces of the fluxes $f^{l, r}(u)$ and of the Kruzhkov entropy fluxes $q^{l, r}(u, k)$ (see [12]).

Remark 2.2. Define the monotone multivalued functions $\left[V^{l}\right]^{-1},\left[V^{l}\right]^{-1}$. Notice that the superpositions $g^{l, r}:=f^{l, r} \circ\left[V^{l, r}\right]^{-1}$ are continuous functions. Moreover, the entropy flux functions $q^{l, r}$, naturally written in terms of the unknown $z$ and of a parameter $k$, can be expressed as continuous functions of $V^{l, r}(z), V^{l, r}(k)$ only. More precisely, we have

$$
\begin{aligned}
q^{l}(z, k) & =\operatorname{sign}(z-k)\left(f^{l}(z)-f^{l}(k)\right) \\
& \equiv \operatorname{sign}\left(V^{l}(z)-V^{l}(k)\right)\left(g^{l} \circ V^{l}(z)-g^{l} \circ V^{l}(k)\right) \\
& =: Q^{l}\left(V^{l}(z), V^{l}(k)\right)
\end{aligned}
$$

a corresponding representation of $q^{r}(\cdot, \cdot)$ by $Q^{r}\left(V^{r}(\cdot), V^{r}(\cdot)\right)$ is valid. 
Remark 2.3. It is easily seen that the traces in the sense of (2.6) can be composed by any continuous function; namely, $\gamma^{l, r}\left(h(V(u))=h\left(\gamma^{l, r} V(u)\right)\right.$ for $h \in C(U ; \mathbb{R})$. It follows that in the context of Theorem 2.1 , there exist strong traces $\gamma^{l, r} q^{l, r}(u, k)$ of the entropy fluxes $q^{l, r}(u, k)$, and these traces are equal to $Q^{l, r}\left(\gamma^{l, r} V^{l, r}(u), V^{l, r}(k)\right)$. This remains true if we replace $k$ by another entropy solution $\hat{u}$. In this case,

$$
\gamma^{l, r} q^{l, r}(u, \hat{u})=Q^{l, r}\left(\gamma^{l, r} V^{l, r}(u), \gamma^{l, r} V^{l, r}(\hat{u})\right) .
$$

Recall that whenever $f^{l, r}$ are non-degenerate in the sense of (2.3), $V^{l, r}$ are just the identity mappings and $Q^{l, r}$ coincide with $q^{l, r}$. Finally, notice that the RankineHugoniot relation for a weak solution $u$ of (1.7) can be expressed under the form $g^{l}\left(\gamma^{l} V^{l}(u)\right)=g^{r}\left(\gamma^{r} V^{r}(u)\right)$.

\section{The model one-dimensional problem}

Consider problem (1.2), (1.7). Here the flux $\mathfrak{f}$ is constant in $x$ on each side of the discontinuity line $\Sigma:=(0, \infty) \times\{0\}$, on which the admissibility condition will be defined. As in Section 2, we denote the Kruzhkov entropy flux by

$$
\mathfrak{q}(x, z, k):=\operatorname{sign}(z-k)(\mathfrak{f}(x, z)-\mathfrak{f}(x, k)) .
$$

We write $q^{l, r}(z, k)$ for the left and right entropy fluxes $\operatorname{sign}(z-k)\left(f^{l, r}(z)-f^{l, r}(k)\right)$. Whenever it is convenient, we represent $f^{l, r}, q^{l, r}$ by means of the continuous functions $g^{l, r}, Q^{l, r}$ and the singular mappings $V^{l, r}$ introduced in Remark 2.2:

$$
f^{l, r}(z)=g^{l, r}\left(V^{l, r} z\right), \quad q^{l, r}(z, k)=Q^{l, r}\left(V^{l, r} z, V^{l, r} k\right),
$$

where, to simplify the notation, we write $V^{l} z$ instead of $V^{l}(z)$, et cetera.

\subsection{Definitions of germs and their basic properties}

Related to the left and right fluxes $f^{l}$ and $f^{r}$, we introduce the following definitions.

Definition 3.1. Any set $\mathcal{G}$ of pairs $\left(u^{l}, u^{r}\right) \in U \times U$ satisfying the RankineHugoniot relation (1.9) is called an admissibility germ (a germ for short). If, in addition, (1.11) holds for all $\left(u^{l}, u^{r}\right),\left(\hat{u}^{l}, \hat{u}^{r}\right) \in \mathcal{G}$, then the germ $\mathcal{G}$ is called an $L^{1}$-dissipative admissibility germ (an $L^{1} D$ germ for short).

In the sequel, we focus on $L^{1} D$ germs, which are those leading to the $L^{1}$-dissipativity properties (1.5), (1.6). In this case, each pair $\left(u^{l}, u^{r}\right) \in \mathcal{G}$ corresponds to a solution of (1.7) of the form (1.10) which will be judged admissible a priori (see also Proposition 3.10(i) below).

According to the analysis carried out in the introduction, if $\left(u^{l}, u^{r}\right) \in \mathcal{G}$ is judged to be an admissible elementary solution on (1.7), then inequality (1.11) together with the Rankine-Hugoniot relation should hold for any other admissible elementary solution $\left(\hat{u}^{l}, \hat{u}^{r}\right)$ of (1.7). Therefore, we introduce the following definition: 
Definition 3.2. Let $\mathcal{G}$ be a germ. The dual germ of $\mathcal{G}$, denoted by $\mathcal{G}^{*}$, is the set of pairs $\left(\hat{u}^{l}, \hat{u}^{r}\right) \in U \times U$ such that (1.11) holds for all $\left(u^{l}, u^{r}\right) \in \mathcal{G}$, and the Rankine-Hugoniot relation $f^{l}\left(\hat{u}^{l}\right)=f^{r}\left(\hat{u}^{r}\right)$ is satisfied.

Hence, each elementary solution of (1.7), which is expected to be admissible, corresponds to a pair $\left(\hat{u}^{l}, \hat{u}^{r}\right) \in \mathcal{G}^{*}$ (see Proposition 3.10(ii) below).

If $\mathcal{G}_{1}, \mathcal{G}_{2}$ are two germs such that $\mathcal{G}_{1} \subset \mathcal{G}_{2}$, we say that $\mathcal{G}_{2}$ is an extension of $\mathcal{G}_{1}$. If both $\mathcal{G}_{1}, \mathcal{G}_{2}$ are $L^{1} D$ germs, we call $\mathcal{G}_{2}$ an $L^{1} D$ extension of $\mathcal{G}_{1}$.

Definition 3.3. If $\mathcal{G}$ is an $L^{1} D$ germ which does not possess a nontrivial $L^{1} D$ extension, then $\mathcal{G}$ is called a maximal $L^{1} D$ germ. If $\mathcal{G}$ is a germ that possesses a unique maximal $L^{1} D$ extension, then $\mathcal{G}$ is called a definite germ.

Notice that any maximal $L^{1} D$ germ is definite. As the following proposition shows, the definiteness of $\mathcal{G}$ is necessary and sufficient for its dual germ $\mathcal{G}^{*}$ to be an $L^{1} D$ germ.

Proposition 3.4. Fix a germ $\mathcal{G}$, and let $\mathcal{G}^{*}$ be the dual germ of $\mathcal{G}$.

(i) One has $\mathcal{G} \subset \mathcal{G}^{*}$ if and only if $\mathcal{G}$ is an $L^{1} D$ germ.

(ii) Assume $\mathcal{G}$ is an $L^{1} D$ germ. Then $\mathcal{G}^{*}$ is the union of all $L^{1} D$ extensions of $\mathcal{G}$. Specifically, $\mathcal{G}$ is a definite germ implies $\mathcal{G}^{*}$ is $\mathcal{G}$ 's unique maximal $L^{1} D$ extension.

(iii) One has $\mathcal{G}^{*}=\mathcal{G}$ if and only if $\mathcal{G}$ is a maximal $L^{1} D$ germ.

(iv) If $\mathcal{G}$ is a definite germ, then $\left(\mathcal{G}^{*}\right)^{*}=\mathcal{G}^{*}$.

(v) If $\mathcal{G}^{*}$ is an $L^{1} D$ germ, then $\mathcal{G}$ is definite.

Proof. Property (i) follows directly from the definitions. For a proof of (ii), let $\mathcal{G}^{\prime}$ be an $L^{1} D$ extension of $\mathcal{G}$. Clearly, $\mathcal{G}^{\prime} \subset \mathcal{G}^{*}$. Reciprocally, let $\left(\hat{u}^{l}, \hat{u}^{r}\right) \in \mathcal{G}^{*}$. Then $\mathcal{G}^{\prime}:=\mathcal{G} \cup\left\{\left(\hat{u}^{l}, \hat{u}^{r}\right)\right\}$ is an $L^{1} D$ extension of $\mathcal{G}$ which contains $\left(\hat{u}^{l}, \hat{u}^{r}\right)$. The second part of assertion (ii) follows immediately. Property (iii) follows from (ii). Properties (ii) and (iii) imply (iv). For a proof of (v), we reason by contradiction. Let $\mathcal{G}_{1}, \mathcal{G}_{2}$ be two different maximal $L^{1} D$ extensions of $\mathcal{G}$. Then $\mathcal{G}_{1} \cup \mathcal{G}_{2}$ is not $L^{1} D$. By (ii), $\mathcal{G}^{*}$ contains $\mathcal{G}_{1} \cup \mathcal{G}_{2}$ and therefore it is not an $L^{1} D$ germ either.

Let $V^{l}, V^{r}$ be the singular mappings introduced in Section 2. By $\operatorname{Ran}\left(V^{l, r}\right)$ we mean the images of $U$ by the functions $V^{l, r}$, respectively. It is clear from (3.2) that the validity of the inequality (1.11) and of the Rankine-Hugoniot condition depends only on $u^{l}, \hat{u}^{l}, u^{r}, \hat{u}^{r}$ through the corresponding values $V^{l} u^{l}, V^{l} \hat{u}^{l}, V^{r} u^{r}, V^{r} \hat{u}^{r}$. This motivates the following definition.

Definition 3.5. Let $\mathcal{G}$ be a germ. The reduced germ of $\mathcal{G}$ is the set

$$
V \mathcal{G}:=\left\{\left(V^{l} u^{l}, V^{r} u^{r}\right) \mid\left(u^{l}, u^{r}\right) \in \mathcal{G}\right\} .
$$

Remark 3.6. The following properties are easily derived:

(i) If $\mathcal{G}$ is a maximal $L^{1} D$ germ, then $\mathcal{G}=\left\{\left(u^{l}, u^{r}\right) \mid\left(V^{l} u^{l}, V^{r} u^{r}\right) \in V \mathcal{G}\right\}$.

(ii) If $\mathcal{G}^{*}$ is the dual of a germ $\mathcal{G}$, then $\mathcal{G}^{*}=\left\{\left(u^{l}, u^{r}\right) \mid\left(V^{l} u^{l}, V^{r} u^{r}\right) \in V \mathcal{G}^{*}\right\}$. 
Remark 3.7. In accordance with (3.2), $V \mathcal{G}^{*}$ can be equivalently defined as the set of pairs $\left(\hat{v}^{l}, \hat{v}^{r}\right) \in \operatorname{Ran}\left(V^{l}\right) \times \operatorname{Ran}\left(V^{r}\right)$ such that the Rankine-Hugoniot condition $g^{l}\left(\hat{v}^{l}\right)=g^{r}\left(\hat{v}^{r}\right)$ holds, and

$$
Q^{l}\left(v^{l}, \hat{v}^{l}\right) \geqq Q^{r}\left(v^{r}, \hat{v}^{r}\right), \quad \text { whenever }\left(v^{l}, v^{r}\right) \in V \mathcal{G} \text {. }
$$

Similarly, if $\mathcal{G}^{*}$ is an $L^{1} D$ germ, then for all $\left(v^{l}, v^{r}\right),\left(\hat{v}^{l}, \hat{v}^{r}\right) \in V \mathcal{G}^{*}$ inequality (3.3) is satisfied. Furthermore, if $\mathcal{G}^{*}$ is a maximal $L^{1} D$ germ, then $V \mathcal{G}^{*}$ does not possess a nontrivial extension $\mathcal{V}^{\prime} \subset \operatorname{Ran}\left(V^{l}\right) \times \operatorname{Ran}\left(V^{r}\right)$ such that (3.3) still holds for all $\left(v^{l}, v^{r}\right),\left(\hat{v}^{l}, \hat{v}^{r}\right) \in \mathcal{V}^{\prime}$.

In the sequel, the reader may assume that $f^{l, r}$ are not constant on any nontrivial interval, so that in all subsequent statements we have $V^{l, r}=\mathrm{Id}, Q^{l, r} \equiv q^{l, r}$, and the reduced germs $V \mathcal{G}, V \mathcal{G}^{*}$ can be replaced with $\mathcal{G}, \mathcal{G}^{*}$, respectively.

\subsection{Definitions and uniqueness of $\mathcal{G}$-entropy solutions}

We are now in a position to define $\mathcal{G}$-entropy solutions of $(1.7),(1.2)$ and study their uniqueness.

Definition 3.8. Let $\mathcal{G}$ be an $L^{1} D$ germ, with dual germ $\mathcal{G}^{*}$. A function $u(t, x)$ in $L^{\infty}\left(\mathbb{R}_{+} \times \mathbb{R} ; U\right)$ is called a $\mathcal{G}$-entropy solution of (1.2), (1.7) if:

(i) the restriction of $u$ to $\Omega^{l}:=\mathbb{R}_{+} \times(-\infty, 0)$ is a Kruzhkov entropy solution of the conservation law with flux $f^{l}(\cdot)$; the restriction of $u$ to $\Omega^{r}:=\mathbb{R}_{+} \times(0, \infty)$ is a Kruzhkov entropy solution of the conservation law with flux $f^{r}(\cdot)$;

(ii) $\mathcal{H}^{1}$-almost everywhere on $\Sigma=(0, \infty) \times\{0\}$, the pair of strong traces $\left(\gamma^{l} V^{l}(u), \gamma^{r} V^{r}(u)\right)$ on $\Sigma$ belongs to the reduced germ $V \mathcal{G}^{*}$ of $\mathcal{G}^{*}$;

(iii) $\mathcal{H}^{1}$-almost everywhere on $\{0\} \times \mathbb{R}$, the trace $\gamma^{0} u$ equals $u_{0}$.

The above definition fits the expectations of the preliminary analysis carried out in Section 1.2. Indeed, notice the following points, which we list in a remark.

Remark 3.9. (i) The existence of strong $L^{1}$ traces $\gamma^{l} V^{l} u, \gamma^{r} V^{r} u, \gamma^{0} u$ in Definition 3.8(ii) is not a restriction: due to Theorem 2.1 and (2.2), it follows from the point (i) of the same definition. Moreover, Definition 3.8(i) and the result of [71] imply that $u$ has a representative in $C\left([0, \infty) ; L_{\text {loc }}^{1}(\mathbb{R})\right)$. In the sequel, we mean that a solution $u$ is defined for all $t$ as an $L^{\infty}(\mathbb{R})$ function.

(ii) A $\mathcal{G}$-entropy solution of (1.2), (1.7) is a weak (distributional) solution.

(iii) $\mathrm{A} \mathcal{G}$-entropy solution possesses both the scaling invariance property (A1) and property (A2) required in Section 1.2.

(iv) In view of Remark 3.6(ii), any function of the form (RPb-sol.) is a $\mathcal{G}$-entropy solution of the Riemann problem (1.7), (1.8) if and only if $\left(u^{l}, u^{r}\right) \in \mathcal{G}^{*}$.

(v) One should compare Definition 3.8(ii) with the definition of admissibility of jumps of $B V$ solutions introduced by VOL'PERT in [84], in the special case $f^{l}=f^{r}$ (see inequality (4.1) in Section 4.2). 
For a proof of Remark 3.9(ii), we notice that Kruzhkov entropy solutions are weak solutions on their domains; thus $u$ is a weak solution on $(0, \infty) \times(\mathbb{R} \backslash\{0\})$. In addition, the Rankine-Hugoniot relation on $\Sigma=\mathbb{R}_{+} \times\{0\}$ holds, because we have $\left(\gamma^{l} V^{l} u, \gamma^{r} V^{r} u\right) \in V \mathcal{G}^{*}$, and Definitions 3.2, 3.5 of $V \mathcal{G}^{*}$ together with Remarks 2.2, 2.3 imply that

$$
\gamma^{l, r} f^{l, r}(u)=\gamma^{l, r} g^{l, r}\left(V^{l, r} u\right)=g^{l, r}\left(\gamma^{l, r} V^{l, r} u\right)=g^{l, r}\left(V^{l, r} w^{l, r}\right)=f^{l, r}\left(w^{l, r}\right),
$$

for some $\left(w^{l}, w^{r}\right) \in \mathcal{G}^{*}$; in particular, $f^{l}\left(w^{l}\right)=f^{r}\left(w^{r}\right)$.

From Remark 3.9(iv) and Proposition 3.4(i), we directly deduce that the germ $\mathcal{G}$ (and, more generally, $\mathcal{G}^{*}$ ) indeed corresponds to a selection of admissible elementary solutions (1.10), as we point out in the succeeding proposition.

Proposition 3.10. (i) If $\left(u^{l}, u^{r}\right) \in \mathcal{G}$, then the function $u:=u^{l} 1_{\{x<0\}}+u^{r} 1_{\{x>0\}}$ is a $\mathcal{G}$-entropy solution of (1.7).

(ii) A function $u(x)$ of the above form is a $\mathcal{G}$-entropy solution of (1.7) if and only if $\left(u^{l}, u^{r}\right) \in \mathcal{G}^{*}$.

Consider the map $S_{t}^{\mathcal{G}}$ which associates to $u_{0} \in L^{\infty}(U)$ the value $u(t, \cdot)$ on the trajectory of some $\mathcal{G}$-entropy solution $u$ defined globally in time. In case such a solution exists, we state that $u_{0}$ belongs to $D$, the domain of $S_{t}^{\mathcal{G}}$. Provided the underlying germ $\mathcal{G}$ is definite, the following theorem shows that such a solution $u$ is necessarily unique; one then deduces that the domain of $S_{t}^{\mathcal{G}}$ is independent of $t$, that $\left(S_{t}^{\mathcal{G}}\right)_{t \geqq 0}$ is a semigroup, and it is strongly continuous in $L_{\text {loc }}^{1}(\mathbb{R})$, according to Definition 3.8(iii). In fact, the theorem shows that the map $S_{t}^{\mathcal{G}}$ is an $L^{1}$-dissipative solver in the sense of Definition 1.1.

Theorem 3.11. Assume that $\mathcal{G}$ is a definite germ. If $u$ and $\hat{u}$ are two $\mathcal{G}$-entropy solutions of problem (1.2), (1.7) corresponding to the initial data $u_{0}$ and $\hat{u}_{0}$ respectively, then the Kato inequality (1.5) and the $L^{1}$-contractivity property (1.6) hold. In particular, there exists at most one $\mathcal{G}$-entropy solution of problem (1.2), (1.7).

Proof. We prove only the Kato inequality; the $L^{1}$-contractivity (1.6) and the uniqueness will follow by considering in (1.5) the test functions $\xi_{R}:=\min \{1,(R-$ $\left.|x|)^{+}\right\}$with $R \rightarrow \infty$, using the continuity of $f^{l}, f^{r}$ and the fact that the space of $x$ has dimension one (see $[17,55])$.

Take $\xi \in \mathcal{D}([0, \infty) \times \mathbb{R}), \xi \geqq 0$. By a standard approximation argument, for $h>0$ we can take the test function $\xi_{h}=\xi \min \left\{1, \frac{(|x|-h)^{+}}{h}\right\}$ in the Kruzhkov entropy formulation, in each of the subdomains $\Omega^{l, r}$. By the "doubling-ofvariables" argument of KRUZHKOV [54], we obtain the standard Kato inequality

$$
-\int_{\mathbb{R}_{+}} \int_{\mathbb{R}}\left\{|u-\hat{u}|\left(\xi_{h}\right)_{t}+\mathfrak{q}(x, u, \hat{u})\left(\xi_{h}\right)_{x}\right\}-\int_{\mathbb{R}}\left|u_{0}-\hat{u}_{0}\right| \xi_{h}(0, x) \leqq 0 .
$$


Clearly, $\xi_{h},\left(\xi_{h}\right)_{t}$ converge to $\xi$, $\xi_{t}$, respectively, in $L^{1}\left(\mathbb{R}_{+} \times \mathbb{R}\right)$. Using Remark 2.2 and calculating $\left(\xi_{h}\right)_{x}$ explicitly, with the Landau notation $\overline{\bar{o}}_{h \rightarrow 0}$, we deduce

$$
\begin{aligned}
& -\int_{\mathbb{R}_{+}} \int_{\mathbb{R}}\left\{|u-\hat{u}| \xi_{t}+\mathfrak{q}(x, u, \hat{u}) \xi_{x}\right\}-\int_{\mathbb{R}}\left|u_{0}-\hat{u}_{0}\right| \xi(0, x)+\overline{\bar{o}}_{h \rightarrow 0}(1) \\
& \quad+\frac{1}{h} \iint_{\mathbb{R}_{+} \times(-2 h,-h)} Q^{l}\left(V^{l}(u), V^{l}(\hat{u})\right) \xi \\
& \quad-\frac{1}{h} \iint_{\mathbb{R}_{+} \times(h, 2 h)} Q^{r}\left(V^{r}(u), V^{r}(\hat{u})\right) \xi \leqq 0
\end{aligned}
$$

Sending $h \rightarrow 0$ in the latter two terms, keeping in mind the definition of strong traces of $V^{l, r}(u), V^{l, r}(\hat{u})$ and bringing into service the continuity of $Q^{l, r}$ [see (2.6) and Remark 2.3], we obtain

$$
\int_{\mathbb{R}_{+}}\left(Q^{l}\left(v^{l}(t), \hat{v}^{l}(t)\right)-Q^{r}\left(v^{r}(t), \hat{v}^{r}(t)\right)\right) \xi(t, 0),
$$

where $v^{l}=\gamma^{l} V^{l} u^{l}, \hat{v}^{l}=\gamma^{l} V^{l} \hat{u}^{l}, v^{r}=\gamma^{r} V^{r} u^{r}$, and $\hat{v}^{r}=\gamma^{r} V^{r} \hat{u}^{r}$ in the pointwise sense for almost every $t>0$. By Definition 3.8(ii), we have $\left(v^{l}(t), v^{r}(t)\right),\left(\hat{v}^{l}(t), \hat{v}^{r}(t)\right) \in V \mathcal{G}^{*}$. Now, because $\mathcal{G}$ is assumed to be definite, by Proposition 3.4 it follows that $\mathcal{G}^{*}$ is an $L^{1} D$ germ. By Remark 3.7 we conclude that the term in (3.5) is nonnegative. Therefore, the Kato inequality (1.5) follows from (3.4) at the limit $h \rightarrow 0$.

We collect a few comments in the next two remarks.

Remark 3.12. (i) The assumption that $\mathcal{G}$ is a definite germ cannot be omitted from the statement of Theorem 3.11, in view of Proposition 3.4(v). Indeed, in the above proof, the fact that $\mathcal{G}^{*}$ is an $L^{1} D$ germ is crucial. An example of non-uniqueness for an $L^{1} D$ germ which is not definite is given in Section 4.7 (Example 4.12).

(ii) Let $\mathcal{G}$ be a definite germ. In view of Proposition 3.4(iv) and Definition 3.8, it follows that $\mathcal{G}$ - and $\mathcal{G}^{*}$-entropy solutions coincide. Certainly, one can choose to work exclusively with maximal $L^{1} D$ germs (in which case $\mathcal{G}^{*} \equiv \mathcal{G}$ ). Yet there can be an advantage in using definite germs $\mathcal{G}$ that are smaller than the corresponding maximal $L^{1} D$ extension $\mathcal{G}^{*}$; see in particular Sections 4, 5, 6.1 , and 6.2.

Remark 3.13. Let $\mathcal{G}$ be a definite germ, and assume $\left(u_{-}, u_{+}\right) \in U \times U$ is such that there exists a $\mathcal{G}$-entropy solution $u$ of the corresponding Riemann problem (1.7), (1.8). Then $u$ is of the form (RPb-sol.) with $\left(u^{l}, u^{r}\right) \in \mathcal{G}^{*}$. In particular, $u$ admits strong left and right traces on $(0, \infty) \times\{0\}$ which are equal to $u^{l}$ and $u^{r}$, respectively. Indeed, the scaling invariance (A1) [see Remark 3.9(iii)] and the uniqueness statement in Theorem 3.11 imply that $u$ is self-similar; by Definition 3.8(i), it follows that $u$ is monotone in the variable $x / t$ on $(-\infty, 0)$ and on $(0, \infty)$. Hence the traces $u^{l, r}:=\gamma^{l, r} u$ exist, and by Remark 3.9(iv), the solution $u$ is of the form (RPb-sol.) with $\left(u^{l}, u^{r}\right) \in \mathcal{G}^{*}$. 
We want next to provide an equivalent definition of solution in which the trace condition of Definition 3.8(ii) is incorporated into the "global" entropy inequalities. As a preparational step, we state the following elementary lemma.

Lemma 3.14. Let $U$ be a closed interval in $\mathbb{R}$ and $f \in C(U ; \mathbb{R})$; for $z, c \in U$, set $q(z, c)=\operatorname{sign}(z-c)(f(z)-f(c))$. Then for all $a, b, c \in U$,

$$
|q(a, c)-q(a, b)| \leqq R(f(\cdot) ; c, b)
$$

where $R(f(\cdot) ; c, b)$ takes on one of the following forms:

- $R(f(\cdot) ; c, b)=2 \operatorname{Osc}(f(\cdot) ; c, b)$, where Osc denotes the oscillation of $f$ on the interval between $c$ and $b$.

$\operatorname{Osc}(f(\cdot) ; c, b):=\max \{|f(z)-f(s)| \mid \min \{c, b\} \leqq z \leqq s \leqq \max \{c, b\}\} ;$

- $R(f(\cdot) ; c, b):=2 \omega(|b-c|)$, where $\omega$ is the modulus of continuity of $f$ on the interval between $c$ and $b$ :

$\omega(h):=\max \{|f(z)-f(s)||\min \{c, b\} \leqq z \leqq s \leqq \max \{c, b\}| z-s \mid, \leqq h$,

- provided $f \in B V_{\mathrm{loc}}(U), R(f(\cdot) ; c, b):=2\left|\int_{c}^{b}\right| f^{\prime}(s)|d s|$ (this is the variation of $f$ on the interval between $c$ and $b$ ).

Definition 3.15. Let $\mathcal{G}$ be an $L^{1} D$ germ. A function $u \in L^{\infty}\left(\mathbb{R}_{+} \times \mathbb{R} ; U\right)$ is called a $\mathcal{G}$-entropy solution of (1.2), (1.7) if it is a weak solution of this problem and for all $\left(c^{l}, c^{r}\right) \in U \times U$,

$$
\begin{aligned}
& \int_{\mathbb{R}_{+}} \int_{\mathbb{R}}\left\{|u(t, x)-c(x)| \xi_{t}+\mathfrak{q}(x, u(t, x), c(x)) \xi_{x}\right\}-\int_{\mathbb{R}}\left|u_{0}(x)-c(x)\right| \xi(0, x) \\
& \quad+\int_{\mathbb{R}_{+}} R_{\mathcal{G}}\left(\left(c^{l}, c^{r}\right)\right) \xi(t, 0) \geqq 0, \quad \forall \xi \in \mathcal{D}([0, \infty) \times \mathbb{R}), \xi \geqq 0,
\end{aligned}
$$

where $c(x)$ is the step function

$$
c(x)=c^{l} 1_{\{x<0\}}+c^{r} 1_{\{x>0\}}= \begin{cases}c^{l}, & x<0, \\ c^{r}, & x>0,\end{cases}
$$

the entropy flux $\mathfrak{q}$ is defined by (3.1), and the remainder term $R_{\mathcal{G}}$ is given by

$$
R_{\mathcal{G}}\left(\left(c^{l}, c^{r}\right)\right):=2 \inf _{\left(b^{l}, b^{r}\right) \in \mathcal{G}}\left(\operatorname{Osc}\left(f^{l}(\cdot) ; c^{l}, b^{l}\right)+\operatorname{Osc}\left(f^{r}(\cdot) ; c^{r}, b^{r}\right)\right)
$$

with the oscillation function Osc defined in (3.7).

Remark 3.16. The explicit requirement that $u$ should be a weak solution is needed only to ensure that the Rankine-Hugoniot condition holds on $\Sigma=(0, \infty) \times\{0\}$. However, for many particular choices of $\mathcal{G}$, a bounded function $u$ satisfying (3.8) is automatically a weak solution of (1.2), (1.7). 
Let us explain the choice of the penalization (remainder) term $R_{\mathcal{G}}\left(\left(c^{l}, c^{r}\right)\right)$ in Definition 3.15, which is inspired by an idea of Отто [67]. The remainder $R_{\mathcal{G}}\left(\left(c^{l}, c^{r}\right)\right)$ is chosen to satisfy the two following properties:

$$
\left(c^{l}, c^{r}\right) \in \mathcal{G} \Rightarrow R_{\mathcal{G}}\left(\left(c^{l}, c^{r}\right)\right)=0,
$$

and (because $\mathcal{G}$ is an $L^{1} D$ germ)

$$
\left(c^{l}, c^{r}\right) \in U \times U,\left(a^{l}, a^{r}\right) \in \mathcal{G} \Rightarrow q^{r}\left(a^{r}, c^{r}\right)-q^{l}\left(a^{l}, c^{l}\right) \leqq R_{\mathcal{G}}\left(\left(c^{l}, c^{r}\right)\right) .
$$

Property (3.10) follows from (3.6) in Lemma 3.14, via the bound

$$
\begin{aligned}
& \text { for all }\left(a^{l}, a^{r}\right),\left(c^{l}, c^{r}\right) \in U \times U, \\
& \inf _{\left(b^{l}, b^{r}\right) \in \mathcal{G}}\left|\left[q^{l}\left(a^{l}, b^{l}\right)-q^{r}\left(a^{r}, b^{r}\right)\right]-\left[q^{l}\left(a^{l}, c^{l}\right)-q^{r}\left(a^{r}, c^{r}\right)\right]\right| \\
& \quad \leqq R_{\mathcal{G}}\left(\left(c^{l}, c^{r}\right)\right),
\end{aligned}
$$

and the inequality $q^{l}\left(a^{l}, b^{l}\right)-q^{r}\left(a^{r}, b^{r}\right) \geqq 0$, which is valid for all $\left(a^{l}, a^{r}\right)$, $\left(b^{l}, b^{r}\right) \in \mathcal{G}$.

According to Lemma 3.14, we can take the remainder term $R_{\mathcal{G}}$ in (3.8) under a few different forms, using, for example the moduli of continuity of the functions $f^{l, r}$ or their variation functions instead of the oscillation functions (3.7). If $f^{l, r}$ are globally Lipschitz continuous on $U$, the simplest choice is to take

$$
\begin{aligned}
R_{\mathcal{G}}\left(\left(c^{l}, c^{r}\right)\right) & :=2\left\|\left(f^{l, r}\right)^{\prime}\right\|_{L^{\infty}} \inf \left\{\left|b^{l}-c^{l}\right|+\left|b^{r}-c^{r}\right| \mid\left(b^{l}, b^{r}\right) \in \mathcal{G}\right\} \\
& \equiv C \operatorname{dist}\left(\left(c^{l}, c^{r}\right), \mathcal{G}\right),
\end{aligned}
$$

where dist is the euclidean distance on $\mathbb{R}^{2}$ and $C$ is a sufficienly large constant. With this choice, the properties (3.9) and (3.10) remain true. See [9] for one application.

The above Definition 3.15 is suitable for many generalizations, including the multi-D setting as in $[10,11]$ and the case of time-dependent families of germs as in [9]. However, for the precise model case (1.7), one can avoid the use of the remainder term $R_{\mathcal{G}}$ in (3.8), thanks to the following result.

Proposition 3.17. The inequalities in (3.8) hold for all $\left(c^{l}, c^{r}\right) \in U \times U$ and for all nonnegative test functions $\xi$, if and only if they hold (with zero remainder term) for the choices

$$
\begin{gathered}
\left\{c^{l}=c^{r}=c \text { with } c \text { arbitrary and }\left.\xi\right|_{x=0}=0\right\} \\
\cup\left\{\left(c^{l}, c^{r}\right) \in \mathcal{G} \text { and } \xi \geqq 0 \text { is arbitrary }\right\} .
\end{gathered}
$$

Proof. According to (3.9), whenever $\left(c^{l}, c^{r}\right) \in \mathcal{G}$, the term $\int_{\mathbb{R}_{+}} R_{\mathcal{G}}\left(\left(c^{l}, c^{r}\right)\right) \xi(t, 0)$ in (3.8) vanishes; clearly, it also vanishes if $\left.\xi\right|_{x=0}=0$. Conversely, following the proof of Theorem 3.18 below, using the test functions $\xi_{h}^{ \pm}$in (3.11) and property (3.10) of the remainder $\mathcal{R}_{\mathcal{G}}$, it is easy to establish (3.8) for all choices of $\left(c^{l}, c^{r}\right)$, $\xi \geqq 0$. 
Using Proposition 3.17, with specific choices of $\mathcal{G}$, from Definition 3.15 we recover the formulations of Baiti and Jenssen [15], Audusse and Perthame [13] (restricted to the model case (1.7)), and BürGER ET AL. [22]; see Section 4 for details. Namely, for test functions $\xi$ which are zero on the interface $\{x=0\}$, the Kruzhkov entropy inequalities with any entropy $\eta(z)=|z-c|, c \in \mathbb{R}$, are required; and for general $\xi$, up-to-the-interface entropy inequalities are required only for a careful selection of "adapted" entropies. ${ }^{4}$ The adapted entropies are $x$-dependent functions of the form

$$
\eta(z, c):=|z-c(x)|, \quad c(x)=c^{l} 1_{\{x<0\}}+c^{r} 1_{\{x>0\}}, \quad\left(c^{l}, c^{r}\right) \in \mathcal{G} .
$$

The notions of solution introduced in Definitions 3.8 and 3.15 bear the same name. Indeed, we have

Theorem 3.18. For any $L^{1} D$ germ $\mathcal{G}$, Definitions 3.8 and 3.15 are equivalent.

Proof. Consider a solution $u$ of (1.2), (1.7) in the sense of Definition 3.8 and fix a pair $\left(c^{l}, c^{r}\right) \in U \times U$; consider now the function $c(x)=c^{l} 1_{\{x<0\}}+c^{r} 1_{\{x>0\}}$. Take $0 \leqq \xi \in \mathcal{D}((0, \infty) \times \mathbb{R})$ and consider the compactly supported in $\Omega^{l, r}$ test functions

$$
\xi_{h}^{ \pm}:=\xi \min \left\{1, \frac{\left(x^{ \pm}-h\right)^{+}}{h}\right\}
$$

in the Kruzhkov entropy formulations of Definition 3.8(i). Since $c(x)$ is constant in each of the domains $\Omega^{l, r}$, setting $\xi_{h}=\xi_{h}^{-}+\xi_{h}^{+}$we have

$$
\int_{\mathbb{R}_{+}} \int_{\mathbb{R}}\left\{|u-c(x)|\left(\xi_{h}\right)_{t}+\mathfrak{q}(x, u, c(x))\left(\xi_{h}\right)_{x}\right\} \geqq 0 .
$$

Sending $h>0$ to zero, using Definition 3.8(i) for the existence of strong traces and Remarks 2.2, 2.3 for some of their properties, we deduce as in (3.4) that

$$
\begin{aligned}
& \int_{\mathbb{R}_{+}} \int_{\mathbb{R}}\left\{|u(t, x)-c(x)| \xi_{t}+\mathfrak{q}(x, u(t, x), c(x)) \xi_{x}\right\} \\
& \quad-\int_{\mathbb{R}_{+}}\left(Q^{l}\left(\left(\gamma^{l} V^{l} u\right)(t), V^{l} c^{l}\right)-Q^{r}\left(\left(\gamma^{r} V^{r} u\right)(t), V^{r} c^{r}\right)\right) \xi(t, 0) \geqq 0 .
\end{aligned}
$$

Set $v^{l, r}(t):=\left(\gamma^{l, r} V^{l, r} u\right)(t)$ and $z^{l, r}:=V^{l, r} c^{l, r}$. By Definition 3.8(ii),

$$
\left(v^{l}(t), v^{r}(t)\right) \in V \mathcal{G}^{*}, \quad \text { for almost every } t>0 .
$$

4 These formulations are very similar to the one introduced by CARRILLO in [26] for homogeneous Dirichlet boundary value problems for degenerate parabolic problems including conservation laws. In our context, we can use the standard Kruzhkov entropies $|z-c|$ in the place of the semi-Kruzhkov entropies $(z-c)^{ \pm}$used by Carrillo. 
Hence, by Remark 3.7, $\forall\left(w^{l}, w^{r}\right) \in V \mathcal{G}$ we obtain $Q^{l}\left(v^{l}, w^{l}\right) \geqq Q^{r}\left(v^{r}, w^{r}\right)$, and so

$$
\begin{aligned}
Q^{l}\left(v^{l}, z^{l}\right)-Q^{r}\left(v^{r}, z^{r}\right)= & Q^{l}\left(v^{l}, w^{l}\right)-Q^{r}\left(v^{r}, w^{r}\right)+Q^{l}\left(v^{l}, z^{l}\right)-Q^{r}\left(v^{r}, z^{r}\right) \\
& -Q^{l}\left(v^{l}, w^{l}\right)+Q^{r}\left(v^{r}, w^{r}\right) \\
\geqq & {\left[Q^{l}\left(v^{l}, z^{l}\right)-Q^{r}\left(v^{r}, z^{r}\right)\right]-\left[Q^{l}\left(v^{l}, w^{l}\right)-Q^{r}\left(v^{r}, w^{r}\right)\right] . }
\end{aligned}
$$

Coming back to the definitions of $V^{l, r}$ and $Q^{l, r}$, from (3.10) we then easily deduce that

$$
Q^{l}\left(v^{l}, z^{l}\right)-Q^{r}\left(v^{r}, z^{r}\right) \geqq-R_{\mathcal{G}}\left(\left(c^{l}, c^{r}\right)\right) ;
$$

therefore (3.12) yields (3.8), at least for test functions $\xi$ that vanish on $\{t=0\}$.

We deduce (3.8) for all $0 \leqq \xi \in \mathcal{D}([0, \infty), \mathbb{R})$, by truncating $\xi$ in a neighbourhood of $\{t=0\}$ and using Definition 3.8(iii). Thanks to Remark 3.9(ii), $u$ is also a weak solution of (1.2), (1.7); thus it is a solution in the sense of Definition 3.15.

Reciprocally, take a solution $u$ of (1.2), (1.7) in the sense of Definition 3.15. First, it is clear that Definition 3.8(i) holds. Therefore it follows from (2.2) that also Definition 3.8(iii) is fulfilled. Similarly, it follows from Theorem 2.1 that strong left and right traces $\gamma^{l, r} V^{l, r} u$ on $\Sigma=(0, \infty) \times\{0\}$ exist. It remains to show that $\left(\left(\gamma^{l} V^{l} u\right)(t),\left(\gamma^{r} V^{r} u\right)(t)\right) \in V \mathcal{G}^{*}$ pointwise on $\Sigma$. To this end, with $\xi \in \mathcal{D}([0, \infty) \times \mathbb{R}), \xi \geqq 0$, and $h>0$, take $\psi_{h}=\frac{(h-|x|)^{+}}{h} \xi$ as test function in (3.8) and pass to the limit as $h \rightarrow 0$. Using Remarks 2.2 and 2.3, we deduce

$$
\int_{\mathbb{R}_{+}}\left(Q^{l}\left(\left(\gamma^{l} V^{l} u\right)(t), V^{l} c^{l}\right)-Q^{r}\left(\left(\gamma^{r} V^{r} u\right)(t), V^{r} c^{r}\right)\right) \xi(t, 0) \geqq 0 .
$$

Now we take $\left(c^{l}, c^{r}\right) \in \mathcal{G}$ and utilize that $R_{\mathcal{G}}\left(\left(c^{l}, c^{r}\right)\right)=0$, see property (3.9). Since $\xi(\cdot, 0) \in \mathcal{D}([0, \infty)), \xi \geqq 0$, is arbitrary, we get

$$
Q^{l}\left(\left(\gamma^{l} V^{l} u\right)(t), V^{l} c^{l}\right) \geqq Q^{r}\left(\left(\gamma^{r} V^{r} u\right)(t), V^{r} c^{r}\right), \text { for almost every } t>0,
$$

for any $\left(c^{l}, c^{r}\right) \in \mathcal{G}$ (one starts with a countable dense subset of $\mathcal{G}$ ). In addition, recall that, $u$ is a weak solution of (1.7); by Remark 2.3, the Rankine-Hugoniot relation $g^{l}\left(\gamma^{l} V^{l} u\right)=g^{r}\left(\gamma^{r} V^{r} u\right)$ holds. By Remark 3.7, we infer that

$$
\left(\left(\gamma^{l} V^{l} u\right)(t),\left(\gamma^{r} V^{r} u\right)(t)\right) \in V \mathcal{G}^{*} .
$$

This justifies Definition 3.8(ii) and concludes the proof.

\subsection{Comparison and continuous dependence results and $L^{\infty}$ estimates}

In this section we provide a comparison result and some $L^{\infty}$ estimates for $\mathcal{G}$-entropy solutions. Moreover, we introduce a "distance" between two germs, which is used to state a continuous dependence result with respect to the choice of the germ $\mathcal{G}$. This result prepares the ground for a study of parametrized families of germs in [10]. 
Theorem 3.19. Assume that $\mathcal{G}$ is a definite germ. Let $u$ and $\hat{u}$ be two $\mathcal{G}$-entropy solutions of problem (1.2), (1.7) corresponding to initial data $u_{0}$ and $\hat{u}_{0}$, respectively, such that $\left(u_{0}-\hat{u}_{0}\right)^{+} \in L^{1}(\mathbb{R})$. Then

$$
\int_{\mathbb{R}}(u-\hat{u})^{+}(t) \leqq \int_{\mathbb{R}}\left(u_{0}-\hat{u}_{0}\right)^{+}, \text {for almost every } t>0 .
$$

In particular, $u_{0} \leqq \hat{u}_{0} \Rightarrow u \leqq \hat{u}$.

Proof. The proof is the same as the one used to conclude Theorem 3.11. The doubling-of-variables argument is used to derive the Kato inequality with $(u-\hat{u})^{+}$ instead of $|u-\hat{u}|$, and we then derive the analogue of inequality (1.11) for the associated entropy fluxes $q_{ \pm}^{l, r}$. As in Remark 2.2, these fluxes are expressed by means of the functions

$$
Q_{ \pm}^{l, r}:(z, k) \mapsto \operatorname{sign}^{ \pm}(z-k)\left(g^{l, r}(z)-g^{l, r}(k)\right) .
$$

Take $\left(v^{l}, v^{r}\right),\left(\hat{v}^{l}, \hat{v}^{r}\right) \in V \mathcal{G}^{*}$; it suffices to show that

$$
Q_{ \pm}^{l}\left(v^{l}, \hat{v}^{l}\right) \geqq Q_{ \pm}^{r}\left(v^{r}, \hat{v}^{r}\right) .
$$

By Remark 3.7, we have (3.3) and, moreover, $g^{l}\left(v^{l}\right)=g^{r}\left(v^{r}\right), g^{l}\left(\hat{v}^{l}\right)=g^{r}\left(\hat{v}^{r}\right)$. Consider for example the case $g^{l}\left(v^{l}\right)-g^{l}\left(\hat{v}^{l}\right)>0$, in which case (3.3) is equivalent to the inequality $\operatorname{sign}\left(v^{l}-\hat{v}^{l}\right) \geqq \operatorname{sign}\left(v^{r}-\hat{v}^{r}\right)$. This implies the following two inequalities: $\operatorname{sign}^{ \pm}\left(v^{l}-\hat{v}^{l}\right) \geqq \overline{\operatorname{sign}}^{ \pm}\left(v^{r}-\hat{v}^{r}\right)$, which in turn imply (3.13). The other cases are similar.

As a corollary, we have the following maximum principle: Let $u$ be a $\mathcal{G}$-entropy solution of (1.7) with an initial function $u_{0}$ satisfying for some $\left(c^{l}, c^{r}\right),\left(C^{l}, C^{r}\right) \in$ $\mathcal{G}^{*}$ the following lower and upper bounds:

$$
\begin{aligned}
c^{l} \leqq u_{0}(x) \leqq C^{l} \quad \text { for almost every } x \in \mathbb{R}_{-}, \\
c^{r} \leqq u_{0}(x) \leqq C^{r} \quad \text { for almost every } x \in \mathbb{R}_{+} .
\end{aligned}
$$

Then, for almost every $t>0$, the same inequalities are satisfied by $u(t, \cdot)$. The proof is immediate from Proposition 3.10(ii) and Theorem 3.19.

In general, $\|u(t, \cdot)\|_{L^{\infty}}$ can be greater than $\left\|u_{0}\right\|_{L^{\infty}}$; see for example the examples in [21]. But we have

Proposition 3.20. Let $\mathcal{G}$ be a definite germ. Let $m_{-} \leqq M_{-}, m_{+} \leqq M_{+}$be real numbers such that there exist $\mathcal{G}$-entropy solutions of the Riemann problems (1.7), (1.8) with data $\left(u_{-}, u_{+}\right)=\left(m_{-}, m_{+}\right)$and with data $\left(u_{-}, u_{+}\right)=\left(M_{-}, M_{+}\right)$. By Remark 3.13, strong left and right traces of these solutions on $\{x=0\}$ exist; denote these traces by $c^{l, r}, C^{l, r}$, respectively. If $m_{ \pm} \leqq u_{0} \leqq M_{ \pm}$almost everywhere on $\mathbb{R}^{ \pm}$and $u$ is the $\mathcal{G}$-entropy solution of (1.7) with the initial function $u_{0}$, then

$$
\begin{aligned}
& \min \left\{m_{-}, c^{l}\right\} \mathbb{1}_{\{x<0\}}+\min \left\{m_{+}, c^{r}\right\} \mathbb{1}_{\{x>0\}} \\
& \quad \leqq u(t, x) \leqq \\
& \max \left\{M_{-}, C^{l}\right\} \mathbb{1}_{\{x<0\}}+\max \left\{M_{+}, C^{r}\right\} \mathbb{1}_{\{x>0\}}, \quad \text { almost everywhere in } \mathbb{R}_{+} \times \mathbb{R} .
\end{aligned}
$$


Proof. The claim follows from the comparison principle in Theorem 3.19 and from the monotonicity property of the solutions of the Riemann problems in the domains $\{x<0\},\{x>0\}$, see Remark 3.13.

Finally, we state a simple result regarding the continuous dependence of $\mathcal{G}$-entropy solutions on the choice of the $L^{1} D$ germ $\mathcal{G}$. For a pair of fixed functions $f^{l, r} \in C(U ; \mathbb{R})$ and the associated Kruzhkov entropy fluxes $q^{l, r}$, consider two $L^{1} D$ germs $\mathcal{G}, \hat{\mathcal{G}}$. Define the "distance" function

$$
\rho(\mathcal{G} ; \hat{\mathcal{G}}):=\max \left\{0, \sup _{\left(b^{l}, b^{r}\right) \in \mathcal{G},\left(\hat{b}^{l}, \hat{b}^{r}\right) \in \hat{\mathcal{G}}}\left(q^{r}\left(b^{r}, \hat{b}^{r}\right)-q^{l}\left(b^{l}, \hat{b}^{l}\right)\right)\right\} .
$$

Observe that $\rho(\mathcal{G} ; \hat{\mathcal{G}})=0$ implies $\mathcal{G} \subset \hat{\mathcal{G}}^{*}$ and $\hat{\mathcal{G}} \subset \mathcal{G}^{*}$. So if the distance $\rho$ between two maximal $L^{1} D$ germs is zero, then the two germs coincide.

Proposition 3.21. With the notation above, let $u, \hat{u}$ be $\mathcal{G}$ - and $\hat{\mathcal{G}}$-entropy solutions of (1.7), respectively, with initial data $u_{0}, \hat{u}_{0}$, respectively. Then

$$
\int_{\mathbb{R}}|u-\hat{u}|(t) \leqq \int_{\mathbb{R}}\left|u_{0}-\hat{u}_{0}\right|+t \rho(\mathcal{G}, \hat{\mathcal{G}}), \quad \text { for almost every } t>0 .
$$

The proof of Proposition 3.21 represents an obvious modification of the last argument of the proof of Theorem 3.11 [namely, the term (3.5) is controlled by the last term on right-hand side of (3.15) when $0 \leqq \xi \leqq 1]$.

\subsection{Complete germs and $\mathcal{G}$-entropy process solutions}

In this section we will briefly discuss the question of existence of $\mathcal{G}$-entropy solutions for a general definite germ $\mathcal{G}$, and introduce the weaker notion of $\mathcal{G}$-entropy process solutions as a tool to establish convergence of approximate solutions equipped with mere $L^{\infty}$ bounds. For some particular cases, existence will be shown in detail in Sections 4 and 6.1, while more general results will be given in Section 6.

Definition 3.22. A germ $\mathcal{G}$ is said to be complete if for all $\left(u_{-}, u_{+}\right) \in U \times U$, the Riemann problem (1.7), (1.8) admits a weak solution of the form (RPb-sol.) with $\left(u^{l}, u^{r}\right) \in \mathcal{G}$ (here and in the sequel, we adopt the convention of Remark 1.2).

Observe that by Proposition 3.4(i) and Remark 3.9(iv), if $\mathcal{G}$ is an $L^{1} D$ germ, then the aforementioned weak solution of problem (1.7), (1.8) is also the unique $\mathcal{G}$-entropy solution of the problem.

Proposition 3.23. Let $\mathcal{G}$ be a definite germ. If there exists a $\mathcal{G}$-entropy solution of (1.2), (1.7) for any $u_{0} \in L^{\infty}(\mathbb{R} ; U)$, then $\mathcal{G}^{*}$ must be complete.

Proof. Consider general Riemann initial data of the form (1.8). By assumption and by Remark 3.13, there exists exactly one $\mathcal{G}$-entropy solution $u$ to (1.7), (1.8); moreover, $u$ is of the form (RPb-sol.), and the corresponding pair of states $\left(u^{l}, u^{r}\right)$ belongs to $\mathcal{G}^{*}$. Since $u$ is necessarily a weak solution, $\mathcal{G}^{*}$ is complete. 
The assumption that $\mathcal{G}^{*}$ is complete is expected to yield existence of $\mathcal{G}$-entropy solutions. Indeed, starting from an appropriate Riemann solver (a classical solver for $x \neq 0$, and the solver $\mathcal{R S}^{\mathcal{G}}$ at $x=0$ ), it is possible to construct numerical approximations of $\mathcal{G}$-entropy solutions, for example by the Godunov finite volume scheme or by the front tracking scheme; see Section 6.3. Unfortunately, currently we can deduce existence only in the presence of good pre-compactness properties (see, for example [22] and [40] for uniform $B V$ estimates for front tracking and adapted Engquist-Osher schemes, respectively).

In passing, we mention that "completeness" makes available uniform $L^{\infty}$ bounds. If $\mathcal{G}$ is definite and $\mathcal{G}^{*}$ is complete, Proposition 3.20 yields an $L^{\infty}$ bound on any $\mathcal{G}$-entropy solution $\mathcal{S}_{t}^{\mathcal{G}} u_{0}$ of the form

$$
\left\|\left(\mathcal{S}_{t}^{\mathcal{G}} u_{0}\right)(\cdot)\right\|_{L^{\infty}(\mathbb{R})} \leqq \text { Const }\left(\left\|u_{0}\right\|_{\left.L^{\infty}, \mathcal{G}\right), \quad \text { uniformly in } t .}\right.
$$

If similar $L^{\infty}$ estimates are available for sequences produced by an approximation procedure, and if the existence of the $\mathcal{G}$-entropy solution is already known by other means, then Definition 3.24 and Theorem 3.28 below provide a "propagation of compactness" approach to proving convergence of such sequences.

To this end, following $[38,39,69,78]$ (see also references cited therein and the previous works of Tartar and DiPerna), we first extend the notion of solution given in Definition 3.15 to account for so-called process solutions (higher dimensional $L^{\infty}$ objects related to the distribution function of the Young measure).

Definition 3.24. Let $\mathcal{G}$ be an $L^{1} D$ germ. A function $\mu \in L^{\infty}\left(\mathbb{R}_{+} \times \mathbb{R} \times(0,1) ; U\right)$ is called a $\mathcal{G}$-entropy process solution of (1.2), (1.7) if the following conditions hold:

1 (weak process formulation). For all $\xi \in \mathcal{D}([0, \infty) \times \mathbb{R})$,

$$
\int_{0}^{1} \int_{\mathbb{R}_{+}} \int_{\mathbb{R}}\left\{\mu(t, x, \alpha) \xi_{t}+\mathfrak{f}(x, \mu(t, x, \alpha)) \xi_{x}\right\}-\int_{\mathbb{R}} u_{0} \xi(0, x)=0 .
$$

2 (penalized entropy process inequalities). For all pairs $\left(c^{l}, c^{r}\right) \in U \times U$ and for all $\xi \in \mathcal{D}([0, \infty) \times \mathbb{R}), \xi \geqq 0$,

$$
\begin{gathered}
\int_{0}^{1} \int_{\mathbb{R}_{+}} \int_{\mathbb{R}}\left\{|\mu(t, x, \alpha)-c(x)| \xi_{t}+\mathfrak{q}(x ; \mu(t, x, \alpha), c(x)) \xi_{x}\right\} \\
-\int_{\mathbb{R}}\left|u_{0}-c(x)\right| \xi(0, x)+\int_{\mathbb{R}_{+}} R_{\mathcal{G}}\left(\left(c^{l}, c^{r}\right)\right) \xi(t, 0) \geqq 0,
\end{gathered}
$$

where $c(x)=c^{l} 1_{\{x<0\}}+c^{r} 1_{\{x>0\}}$, and the remainder term $R_{\mathcal{G}}\left(\left(c^{l}, c^{r}\right)\right)$ has the same meaning as in Definition 3.15.

As for Definition 3.15, alternative (equivalent) forms of the remainder term can be chosen, provided the two properties (3.9) and (3.10) are fulfilled.

Remark 3.25. (see Proposition 3.17) A $\mathcal{G}$-entropy process solution is equivalently characterized by the following three requirements: 
(a) The weak process formulation (3.16) holds;

(b) For all $\xi \in \mathcal{D}([0, \infty) \times \mathbb{R}), \xi \geqq 0$, such that $\xi=0$ on the interface $\{x=0\}$, for all pairs $(c, c), c \in U$, the entropy inequalities (3.17) hold (with zero remainder term);

(c) For all $\xi \in \mathcal{D}([0, \infty) \times \mathbb{R})$, $\xi \geqq 0$, for all pairs $\left(c^{l}, c^{r}\right) \in \mathcal{G}$, the entropy inequalities (3.17) hold (with zero remainder term).

Indeed, it is evident that Definition 3.24 implies the three properties above. Clearly, in the cases $(b)$ and $(c)$ above, the remainder in (3.17) vanishes, see (3.9). For the proof of the converse implication, as in the proof of Theorem 3.18, we utilize the test functions $\xi_{h}^{ \pm}$given in (3.11) and the remainder term property (3.10).

Remark 3.26. Fix a pair $\left(c^{l}, c^{r}\right) \in U \times U$, and let $\mu$ be a $\mathcal{G}$-entropy process solution. We want to consider the functions

$$
\begin{gathered}
I^{l, r}:(t, x) \mapsto \int_{0}^{1}\left|\mu(t, x, \alpha)-c^{l, r}\right| d \alpha, \\
J^{l, r}:(t, x) \mapsto \int_{0}^{1} q^{l, r}\left(\mu(t, x, \alpha), c^{l, r}\right) d \alpha .
\end{gathered}
$$

The inequalities in (3.17) imply that the $\mathbb{R}^{2}$ vector field

$$
(t, x) \mapsto\left(I^{l}(t, x), J^{l}(t, x)\right)
$$

is an $L^{\infty}$ divergence-measure field on $\mathbb{R}_{+} \times(-\infty, 0)$ (locally in $\left.t\right)$. According to the results in CHEN and FRID [29], such a vector field admits a weak normal trace on the boundary $\Sigma=\mathbb{R}_{+} \times\{0\}$. This means that $J^{l}$ admits a weak trace from the left on $\Sigma$. Similarly, we conclude that $J^{r}$ admits a weak trace from the right on $\Sigma$.

In what follows, the weak trace operators $t \mapsto \gamma_{w}^{l, r} J^{l, r}(t, \cdot)$ from Remark 3.26 will be denoted by $\gamma_{w}^{l, r}$.

Lemma 3.27. Let $\mu$ be a $\mathcal{G}$-entropy process solution. Then for all $\left(v^{l}, v^{r}\right) \in V \mathcal{G}$,

$$
\gamma_{w}^{l} \int_{0}^{1} Q^{l}\left(V^{l} \mu(t, \cdot, \alpha), v^{l}\right) d \alpha \geqq \gamma_{w}^{r} \int_{0}^{1} Q^{r}\left(V^{r} \mu(t, \cdot, \alpha), v^{r}\right) d \alpha,
$$

for almost every $t>0$.

Proof. The proof is very similar to the proof of the second part of Theorem 3.18. We take $c^{l, r}$ such that $v^{l, r}=V^{l, r} c^{l, r}$. For $\xi \in \mathcal{D}([0, \infty) \times \mathbb{R}), \xi(\cdot, 0) \geqq 0$, and $h>0$, we take $\frac{(h-|x|)^{+}}{h} \xi$ as test function in (3.17), use the Fubini theorem, and pass to the limit as $h \rightarrow 0$ to finally arrive at (3.18).

Notice that, unlike Remark 3.12(ii), it is not clear whether $\mathcal{G}$ - and $\mathcal{G}^{*}$ - entropy process solutions coincide for a definite germ $\mathcal{G}$. Therefore we require that $\mathcal{G}=\mathcal{G}^{*}$ (that is, we require that $\mathcal{G}$ is a maximal $L^{1} D$ germ) while working with $\mathcal{G}$-entropy process solutions. 
Theorem 3.28. Let $\mathcal{G}$ be a maximal $L^{1} D$ germ. Suppose $u_{0}$ is such that there exists $a \mathcal{G}$-entropy solution $u$ of (1.7) with initial data (1.2). Then there exists a unique $\mathcal{G}$-entropy process solution $\mu$ with initial data $u_{0}$, and $\mu(\alpha)=u$ for almost every $\alpha \in(0,1)$.

Proof. Being a $\mathcal{G}$-entropy solution, $u$ fulfills Definition 3.8(ii). Since $\mathcal{G}=\mathcal{G}^{*}$ by Proposition 3.4(iii), the pair $\left(v^{l}(t), v^{r}(t)\right):=\left(\left(\gamma^{l} V^{l} u\right)(t),\left(\gamma^{r} V^{r} u\right)(t)\right)$ belongs to $V \mathcal{G}^{*}$ for almost every $t>0$. Hence, inequality (3.18) at a point $t>0$ holds in particular with the choice $v^{l, r}=v^{l, r}(t)$. Further, notice that since the traces of $V^{l, r} u$ are strong, the weak traces $\gamma_{w}^{l, r} \int_{0}^{1} Q^{l, r}\left(V^{l, r} \mu(\alpha), V^{l, r} u\right) d \alpha$ on $\Sigma$ exist and are equal to $\gamma_{w}^{l, r} \int_{0}^{1} Q^{l, r}\left(V^{l, r} \mu(\alpha), \gamma^{l, r} V^{l, r} u\right) d \alpha$. Therefore

$$
\begin{aligned}
& \int_{\mathbb{R}_{+}}\left(\gamma_{w}^{l} \int_{0}^{1} Q^{l}\left(V^{l} \mu(t, \cdot, \alpha), V^{l} u(t, \cdot)\right) d \alpha\right. \\
& \left.-\gamma_{w}^{r} \int_{0}^{1} Q^{r}\left(V^{r} \mu(t, \cdot, \alpha), V^{r} u(t, \cdot)\right) d \alpha\right) \xi(t, 0) \geqq 0,
\end{aligned}
$$

for all $\xi(\cdot, 0) \in \mathcal{D}([0, \infty)), \xi(\cdot, 0) \geqq 0$. Now we repeat the arguments in the proof of Theorem 3.11, which leads to the analogue of inequality (3.4); after that, in view of (3.19), we deduce

$$
\int_{0}^{1} \int_{\mathbb{R}_{+}} \int_{\mathbb{R}}\left\{|u-\mu(\alpha)| \xi_{t}+\mathfrak{q}(x, u, \mu(\alpha)) \xi_{x}\right\} \geqq 0,
$$

because $u$ and $\mu$ are solutions with the same data. As in [54] (for Lipschitz fluxes $f^{l, r}$ ) or in $[17,55]$ (for merely continuous $f^{l, r}$ ), we work out finally that

$$
\mu(\cdot, \cdot, \alpha)=u(\cdot, \cdot) \text { almost everywhere on } \mathbb{R}_{+} \times \mathbb{R} \text {, for any } \alpha \in(0,1) .
$$

We have the following open problem: Is it true that for a definite germ $\mathcal{G}$ there is at most one $\mathcal{G}$-entropy process solution $\mu$ with given initial data $u_{0}$ (in which case $\mu$ is independent of $\alpha$ ) ? The answer is known to be positive in some situations. Firstly, according to $[38,39]$, this is the case for the Kruzhkov entropy process solutions of $u_{t}+f(u)_{x}=0$, which can be equivalently defined by Definition 3.24 starting from the germ $\mathcal{G}_{V K r}$ (see Section 4.2). Secondly, if $f^{l}$ is non-decreasing and $f^{r}$ is non-increasing, then every $L^{1} D$ germ $\mathcal{G}$ is a singleton $\left\{\left(v^{l}, v^{r}\right)\right\}$ (see Section 4.5); in this case, from (3.18) we readily derive that $V^{l, r} \mu(\alpha)=v^{l, r}$ for almost every $\alpha$, and thus uniqueness of a $\mathcal{G}$-entropy solution follows.

\subsection{Conclusions}

Let us review the results of Section 3. Admissibility of entropy solutions to the model equation (1.7) is most naturally defined starting from a germ $\mathcal{G}$ possessing a unique maximal $L^{1} D$ extension $\mathcal{G}^{*}$ that is complete. In this case, uniqueness, $L^{1}$-contractivity, and comparison properties hold for solutions in the sense of (the 
equivalent) Definitions 3.8 and 3.15, and we can hope for the existence of solutions for general data. Furthermore, uniform $L^{\infty}$ estimates of solutions can be obtained (see, for example Proposition 3.20) and measure-valued solutions (see Definition 3.24) can be considered, but for the latter we need to know already the existence of solutions in the sense of Definitions 3.8, 3.15 and also the additional assumption $\mathcal{G}=\mathcal{G}^{*}$ must hold; under these conditions we are able to conclude that Definition 3.24 is in fact equivalent to Definitions 3.8, 3.15. In Sections 4, 5, we illustrate the theory of germs by a number of examples.

Remark 3.29. Each maximal $L^{1} D$ germ $\mathcal{G}$ gives rise to an $L^{1}$-contractive semigroup for (1.7). Conversely, by the analysis in Section 1.2, any semigroup defined on the whole space $L^{\infty}(\mathbb{R} ; U)$ that satisfy (A1) and (A2) corresponds to a complete maximal $L^{1} D$ germ. If we drop the semigroup property and the scaling invariance (A1), then different $L^{1}$-dissipative solvers can be constructed, for example starting from a family $\{\mathcal{G}(t)\}_{t>0}$ of maximal $L^{1} D$ germs; we refer to Colombo and GoATIN [31] for an example of such a solver, and to [9] for an analysis of the ColomboGoatin solver in terms of admissibility germs. Notice that in order to have existence we must assume that $\mathcal{G}(\cdot)$ is measurable in an appropriate sense. We will develop an approach to measurability for time-dependent families of germs in [10].

\section{Examples and analysis of known admissibility criteria}

In this section we first discuss the completeness of germs, a "closure" operation on germs, and relations between completeness and maximality of $L^{1} D$ germs. We continue by reviewing a number of known admissibility criteria for problem (1.7) and make explicit the underlying germs. In some cases, using the results of Section 3, we refine the known uniqueness theory. Some known and some new existence results for these criteria are contained in Sections 6.2, 6.3. A similar study of the important "vanishing viscosity" germ is postponed to Sections 5 and 6.1.

\subsection{More about complete germs and closed germs}

For a given $u^{l} \in U$, we denote by $\theta^{l}\left(\cdot, u^{l}\right)$ the graph that contains all points $\left(u_{-}, f^{l}\left(u_{-}\right)\right) \in U \times \mathbb{R}$ such that there exists a Kruzhkov solution to the Riemann problem joining the state $u_{-}$at $x<0, t=0$ to the state $u^{l}$ at $x=0^{-}, t>0$ (that is, this solution contains only waves of nonpositive speed). Similarly, for $u^{r} \in U$, $\theta^{r}\left(\cdot, u^{r}\right)$ is the graph of all points $\left(u_{+}, f^{r}\left(u_{+}\right)\right)$such that there exists a Kruzhkov solution to the Riemann problem joining $u^{r}$ at $x=0^{+}, t>0$, to $u_{+}$at $x>0$, $t=0$ (that is, this solution contains only waves of nonnegative speed).

Note that in concrete situations, the completeness of a given germ can be checked as follows.

Remark 4.1. A germ $\mathcal{G}$ is complete if and only if

$$
\bigcup_{\left(u^{l}, u^{r}\right) \in \mathcal{G}} \operatorname{Dom} \theta^{l}\left(\cdot, u^{l}\right) \times \operatorname{Dom} \theta^{r}\left(\cdot, u^{r}\right)=U \times U .
$$


According to the convention of Remark 1.2, the pair of traces $\left(\gamma^{l} u, \gamma^{r} u\right)$ of a solution of the form (RPb.-sol) does not necessarily coincide with $\left(u^{l}, u^{r}\right)$. The reason is that zero-speed shocks may be, at least in principle, a part of the wave fans joining $u_{-}$to $u^{l}$ and $u^{r}$ to $u^{+}$. In order to cope with such situations, we introduce the following definition.

Definition 4.2. A left (respectively, right) contact shock is a pair $\left(u_{-}, u^{l}\right) \in U \times U$ (respectively, $\left.\left(u^{r}, u_{+}\right) \in U \times U\right)$ such that $u(x)=\left\{\begin{array}{ll}u_{-}, & x<0, \\ u^{l}, & x>0\end{array}\right.$ is a Kruzhkov entropy solution of $u_{t}+f^{l}(u)_{x}=0$ in $\mathbb{R}_{+} \times \mathbb{R}$ (respectively, such that $u(x)=\left\{\begin{array}{ll}u^{r}, & x<0, \\ u_{+}, & x>0,\end{array}\right.$ is a Kruzhkov entropy solution of $u_{t}+f^{r}(u)_{x}=0$ in $\left.\mathbb{R}_{+} \times \mathbb{R}\right)$.

In other words,

$\left(u_{-}, u^{l}\right)$ is a left contact shock $\Longleftrightarrow u_{-} \in \operatorname{Dom} \theta^{l}\left(\cdot, u^{l}\right)$ and $f^{l}\left(u_{-}\right)=f^{l}\left(u^{l}\right)$;

an analogous statement is true for the right contact shocks.

Clearly, any pair $(c, c) \in U \times U$ is both a left and a right contact shock.

Definition 4.3. A germ $\mathcal{G}$ is said to be closed, if $\mathcal{G}$ is a closed subset of $U \times U$ and, moreover, for all pairs $\left(u^{l}, u^{r}\right) \in \mathcal{G}, \mathcal{G}$ also contains all pairs $\left(u_{-}, u_{+}\right)$such that $\left(u_{-}, u^{l}\right)$ is a left contact shock, $\left(u^{r}, u_{+}\right)$is a right contact shock. The smallest closed extension of $\mathcal{G}$ is called the closure of $\mathcal{G}$ and is denoted by $\overline{\mathcal{G}}$.

It is clear that for any solution $u$ of the form $\left(\mathrm{RPb}\right.$.-sol) such that $\left(u^{l}, u^{r}\right) \in \mathcal{G}$, we have $\left(\gamma^{l} u, \gamma^{r} u\right) \in \overline{\mathcal{G}}$ (see the convention of Remark 1.2).

The above definition is consistent in the sense that the closure $\overline{\mathcal{G}}$ of a germ $\mathcal{G}$ is indeed a germ. Indeed, the Rankine-Hugoniot condition for $\left(u_{-}, u_{+}\right) \in \overline{\mathcal{G}}$ holds because it holds for the corresponding pair $\left(u^{l}, u^{r}\right) \in \mathcal{G}$.

Let us list some properties of the closure operation and of closed germs (to be utilized in Section 5).

\section{Proposition 4.4.}

(i) $\mathcal{G}$ is an $L^{1} D$ germ $\Rightarrow \overline{\mathcal{G}}$ is an $L^{1} D$ germ. Furthermore, $\overline{\mathcal{G}} \subset \mathcal{G}^{*},(\overline{\mathcal{G}})^{*}=\mathcal{G}^{*}$.

(ii) A maximal $L^{1} D$ germ $\mathcal{G}$ is closed.

(iii) Any maximal $L^{1} D$ extension of $\mathcal{G}$ contains $\overline{\mathcal{G}}$. In particular, $\mathcal{G}$ is a definite germ if and only if $\overline{\mathcal{G}}$ is a definite germ.

(iv) If $\mathcal{G}$ is a definite germ, then $\mathcal{G}$ and $\overline{\mathcal{G}}$ entropy solutions coincide.

Proof. (i) Fix $\left(u^{l}, u^{r}\right),\left(\hat{u}^{l}, \hat{u}^{r}\right) \in \mathcal{G}$, and let $\left(u_{-}, u^{l}\right),\left(\hat{u}_{-}, \hat{u}^{l}\right)$ be left contact shocks and $\left(u^{r}, u_{+}\right),\left(\hat{u}^{r}, \hat{u}_{+}\right)$be right contact shocks. The Kruzhkov admissibility of these shocks implies that

$$
q^{l}\left(u_{-}, \hat{u}_{-}\right) \geqq q^{l}\left(u^{l}, \hat{u}^{l}\right), \quad q^{r}\left(u^{r}, \hat{u}^{r}\right) \geqq q^{r}\left(u_{+}, \hat{u}_{+}\right) .
$$

Moreover, we have $q^{l}\left(u^{l}, \hat{u}^{l}\right) \geqq q^{r}\left(u^{r}, \hat{u}^{r}\right)$ because $\mathcal{G}$ is an $L^{1} D$ germ. Therefore, we infer $q^{l}\left(u_{-}, \hat{u}_{-}\right) \geqq q^{r}\left(u_{+}, \hat{u}_{+}\right)$. From the definition, it is not difficult to see that $\overline{\mathcal{G}}$ is obtained by the following two operations: 
- firstly, taking the topological closure in $\mathbb{R}^{2}$, and

- secondly, adjoining (possibly trivial) left and right contact shocks to the elements of $\mathcal{G}$.

The result of the second operation is topologically closed. Because each of the two operations preserves inequality (1.11), by the argument above and by the continuity of $q^{l, r}, \overline{\mathcal{G}}$ is an $L^{1} D$ germ. By Proposition 3.4(ii), $\overline{\mathcal{G}}$ being an $L^{1} D$ extension of $\mathcal{G}$, $\overline{\mathcal{G}}$ is contained within $\mathcal{G}^{*}$.

Finally, by Definition 3.2, $\mathcal{G} \subset \overline{\mathcal{G}}$ implies $(\mathcal{G})^{*} \supset(\overline{\mathcal{G}})^{*}$. The reciprocal inclusion is also true. Indeed, by Proposition 3.4(ii), each pair $\left(u^{l}, u^{r}\right) \in \mathcal{G}^{*}$ belongs to an $L^{1} D$ extension $\mathcal{G}^{\prime}$ of $\mathcal{G}$. By Definition $4.3, \mathcal{G} \subset \mathcal{G}^{\prime}$ implies $\overline{\mathcal{G}} \subset \overline{\mathcal{G}^{\prime}}$. By our first claim, $\overline{\mathcal{G}^{\prime}}$ is an $L^{1} D$ extension of $\overline{\mathcal{G}}$; using once more Proposition 3.4(ii), we infer that $\left(u^{l}, u^{r}\right) \in \overline{\mathcal{G}^{\prime}} \subset(\overline{\mathcal{G}})^{*}$. Thus $\mathcal{G}^{*}=(\overline{\mathcal{G}})^{*}$.

(ii) By Definition 4.3 and by (i), one has $\mathcal{G} \subset \overline{\mathcal{G}} \subset \mathcal{G}^{*}$. Since $\mathcal{G}=\mathcal{G}^{*}$ by Proposition 3.4(iii), it follows that $\mathcal{G}$ coincides with its closure.

(iii) If $\mathcal{G} \subset \mathcal{G}^{\prime}$ and $\mathcal{G}^{\prime}$ is a maximal $L^{1} D$ germ, then $\overline{\mathcal{G}} \subset \overline{\mathcal{G}^{\prime}}$; besides, $\overline{\mathcal{G}^{\prime}}=\mathcal{G}^{\prime}$ by property (ii).

(iv) This follows from (i),(iii) and Remark 3.12(ii).

Let us list separately the properties related to complete germs.

Proposition 4.5. (i) If $\mathcal{G}$ is a complete $L^{1} D$ germ, then $\overline{\mathcal{G}}$ is a maximal $L^{1} D$ germ.

(ii) If $\mathcal{G}$ is an $L^{1} D$ germ such that $\overline{\mathcal{G}}$ is complete, then $\mathcal{G}$ is definite and $\mathcal{G}^{*}=\overline{\mathcal{G}}$.

Notice that in case (ii), $\mathcal{G}$ is a definite $L^{1} D$ germ and $\mathcal{G}^{*}$ is complete; as it is pointed out in Section 3.5, such germs are expected to lead to a well-posedness theory for $\mathcal{G}$-entropy solutions.

Proof. (i) Let $\mathcal{G}^{\prime}$ be a nontrivial maximal $L^{1} D$ extension of $\overline{\mathcal{G}}$, and pick a pair $\left(u_{-}, u_{+}\right) \in \mathcal{G}^{\prime} \backslash \overline{\mathcal{G}}$. Now the Riemann problem (1.7), (1.8) possesses two solutions of the form ( $\mathrm{RPb}$.-sol), namely

- a solution $u=\mathcal{R} \mathcal{S}^{\mathcal{G}}\left(u_{-}, u_{+}\right)$, which exists because $\mathcal{G}$ is complete;

- the elementary solution $\hat{u}=u_{-} \mathbb{1}_{\{x<0\}}+u_{+} \mathbb{1}_{\{x>0\}}$.

Both are $\mathcal{G}^{\prime}$-entropy solutions, by Remark 3.9(iv) and because $\left(\gamma^{l} u, \gamma^{r} u\right) \in \overline{\mathcal{G}} \subset$ $\mathcal{G}^{\prime}=\left(\mathcal{G}^{\prime}\right)^{*}$ and $\left(\gamma^{l} \hat{u}, \gamma^{r} \hat{u}\right)=\left(u_{-}, u_{+}\right) \in \mathcal{G}^{\prime}=\left(\mathcal{G}^{\prime}\right)^{*}$. In addition, $u$ and $\hat{u}$ do not coincide almost everywhere because their traces are different: $\left(\gamma^{l} \hat{u}, \gamma^{r} \hat{u}\right)=$ $\left(u_{-}, u_{+}\right) \notin \overline{\mathcal{G}} \ni\left(\gamma^{l} u, \gamma^{r} u\right)$. Since $\mathcal{G}^{\prime}$ is maximal $L^{1} D$, this contradicts the uniqueness result of Theorem 3.11. This contradiction proves that $\overline{\mathcal{G}}$ is itself maximal $L^{1} D$.

(ii) By Proposition 4.4(i), $\overline{\mathcal{G}}$ is a complete $L^{1} D$ germ. By (i), it is a maximal $L^{1} D$ germ and thus it is definite. By Proposition 4.4(iii), also $\mathcal{G}$ is definite. Finally, by Propositions 4.4(i) and 3.4(iii), it follows that $\mathcal{G}^{*}=(\overline{\mathcal{G}})^{*}=\overline{\mathcal{G}}$.

Finally, let us point out a situation where the existence of a specific pair in an $L^{1} D$ germ $\mathcal{G}$ immediately excludes some other pairs from $\mathcal{G}$. 
Proposition 4.6. Let $\mathcal{G}$ be an $L^{1} D$ germ, and fix a pair $\left(u^{l}, u^{r}\right) \in \mathcal{G}$. Consider $u_{-} \in \operatorname{Dom} \theta^{l}\left(\cdot, u^{l}\right)$ and $u_{+} \in \operatorname{Dom} \theta^{r}\left(\cdot, u^{r}\right)$. Then $\left(u_{-}, u_{+}\right) \notin \overline{\mathcal{G}}$, except in the case with $f^{l}\left(u_{-}\right)=f^{r}\left(u_{+}\right)=f^{l}\left(u^{l}\right)=f^{r}\left(u^{r}\right)$.

Proof. By Proposition 4.4(i), $\overline{\mathcal{G}}$ is an $L^{1} D$ germ. Since $\left(u^{l}, u^{r}\right) \in \overline{\mathcal{G}}$, in case $\left(u_{-}, u_{+}\right) \in \overline{\mathcal{G}}$ we must have $f^{l}\left(u_{-}\right)=f^{r}\left(u_{+}\right)$and $q^{l}\left(u_{-}, u^{l}\right) \geqq q^{r}\left(u_{+}, u^{r}\right)$. But the monotonicity of $\theta^{l, r}\left(\cdot, u^{l, r}\right)$ (which is easy to get from the description of the Kruzhkov solutions of the Riemann problem, see, for example [27,44]) yields

$$
\begin{gathered}
q^{l}\left(u_{-}, u^{l}\right)=\operatorname{sign}\left(u_{-}-u^{l}\right)\left(f^{l}\left(u_{-}\right)-f^{l}\left(u^{l}\right)\right)=-\left|f^{l}\left(u_{-}\right)-f^{l}\left(u^{l}\right)\right|, \\
q^{r}\left(u_{+}, u^{r}\right)=\operatorname{sign}\left(u_{+}-u^{r}\right)\left(f^{r}\left(u_{+}\right)-f^{r}\left(u^{r}\right)\right)=\left|f^{r}\left(u_{+}\right)-f^{r}\left(u^{r}\right)\right| .
\end{gathered}
$$

These conditions are not compatible, unless $f^{l}\left(u_{-}\right)=f^{r}\left(u_{+}\right)=f^{l}\left(u^{l}\right)=$ $f^{r}\left(u^{r}\right)$. In addition, if the latter condition holds, then $\left(u_{-}, u^{l}\right)$ (respectively, $\left(u^{r}, u_{+}\right)$is a left (respectively, right) contact shock; in which case $\left(u_{-}, u_{+}\right) \in \overline{\mathcal{G}}$.

\subsection{The case $f^{l} \equiv f^{r}$; the Vol'pert-Kruzhkov germ}

We will now illustrate the abstract "discontinuous flux" theory on a well-known example, namely the case without a flux discontinuity at $\{x=0\}$, so $f^{l}=f^{r}$ in (1.7); let us write $f$ instead of $f^{l, r}$ and take $U=\mathbb{R}$.

In this case, the set of weak solutions of (1.7) includes all constant in $\mathbb{R}_{+} \times \mathbb{R}$ solutions. Let us postulate that all constants are admissible elementary solutions of $u_{t}+f(u)_{x}=0$; in other words, consider the "Vol'pert-Kruzhkov" germ

$$
\mathcal{G}_{V K r}:=\{(c, c) \mid c \in \mathbb{R}\} .
$$

One checks immediately that $\mathcal{G}$ is an $L^{1} D$ germ. According to the definition, the dual germ $\mathcal{G}_{V K r}^{*}$ is determined by the family of inequalities:

$$
\left(u^{l}, u^{r}\right) \in \mathcal{G}_{V K r}^{*} \Longleftrightarrow q\left(u^{l}, c\right) \geqq q\left(u^{r}, c\right), \quad \forall c \in \mathbb{R}
$$

(here $q(u, c)=\operatorname{sign}(u-c)(f(u)-f(c)))$. Indeed, the Rankine-Hugoniot condition $f\left(u^{l}\right)=f\left(u^{r}\right)$, which enters Definition 3.2, follows by taking $\pm c$ large enough in (4.1). Following VoL'PERT [84], we check that $\mathcal{G}_{V K r}^{*}$ is an $L^{1} D$ germ:

$$
q\left(u^{l}, \hat{u}^{l}\right) \geqq q\left(u^{r}, \hat{u}^{l}\right)=q\left(\hat{u}^{l}, u^{r}\right) \geqq q\left(\hat{u}^{r}, u^{r}\right)=q\left(u^{r}, \hat{u}^{r}\right),
$$

for all $\left(u^{l}, u^{r}\right),\left(\hat{u}^{l}, \hat{u}^{r}\right) \in \mathcal{G}_{V K r}^{*}$. By Proposition 3.4(v), $\mathcal{G}_{V K r}$ is definite. Therefore uniqueness of $\mathcal{G}_{V K r}$-entropy solutions holds.

Moreover, it is well known (see, for example [41] and [27,44]) that there exists a Kruzhkov entropy solution of $u_{t}+f(u)_{x}=0$ with the Riemann data (1.8), and that this solution is of the form (RPb.-sol) with $\left(u^{l}, u^{r}\right)$ satisfying (4.1). Therefore the germ $\mathcal{G}_{K r}^{*}$ is also complete, which raises the hope for existence of $\mathcal{G}_{V K r}$-entropy solutions for all bounded data. Not surprisingly, of course, it easily follows that Definition 3.15 with $\mathcal{G}=\mathcal{G}_{V K r}$ is equivalent to the definition of Kruzhkov entropy solutions. Hence, the existence of $\mathcal{G}_{V K r}$-entropy solutions is a consequence of [54]. 
In conclusion, the germ $\mathcal{G}_{V K r}$ leads to a complete well-posedness theory for solutions in the sense of Definitions 3.8 and 3.15, which turns out to be precisely the theory of Kruzhkov entropy solutions. Another description of the associated maximal $L^{1} D$ germ $\mathcal{G}_{V K r}^{*}$ is given in Section 4.3 below.

Remark 4.7. As a final remark, this example is somewhat misleading. Indeed, we have just investigated the question of admissibility of discontinuities of solutions of $u_{t}+f(u)_{x}=0$ on the line $\{x=0\}$; and we took for granted that the admissibility of $u$ on $\{x<0\}$ and $\{x>0\}$ is understood in the sense of Kruzhkov. The focus on the line $\{x=0\}$ is "artificial" in this example. The fact that we finally arrive at notion of Kruzhkov entropy solutions on the whole of $\mathbb{R}_{+} \times \mathbb{R}$ is of course expected; moreover, our existence analysis relies on the knowledge of the Riemann solver associated with Kruzhkov entropy solutions! But we believe that this example and its sequel in Section 4.3 illustrate well the general approach and some techniques related to the use of admissibility germs. Also see Section 4.9 and Remark 4.8 for examples of non-Kruzhkov $L^{1}$-dissipative solvers for $u_{t}+f(u)_{x}=0$.

\subsection{The case $f^{l} \equiv f^{r}$; the Gelfand germ}

We will continue with the above example but apply a different approach, which will be extensively investigated in Section 5 in the case of a discontinuous flux.

Recall that existence of Kruzhkov entropy solutions for

$$
u_{t}+f(u)_{x}=0,\left.\quad u\right|_{t=0}=u_{0}
$$

was first obtained in [54] through the limit, as $\varepsilon \downarrow 0$, of the vanishing viscosity approximations

$$
u_{t}+f(u)_{x}=\varepsilon u_{x x} .
$$

Indeed, it is well known that there exists a unique solution $u^{\varepsilon}$ to (4.3) for all bounded data, that the solutions satisfy an analogue of the $L^{1}$-dissipativity property (1.5) for each $\varepsilon>0$, and that for any $u_{0} \in L^{\infty},\left(u^{\varepsilon}\right)_{\varepsilon>0}$ is relatively compact in $L_{\text {loc }}^{1}$.

Using diagonal, density, and comparison arguments (see Section 6.1 below for details), at the limit $\varepsilon \rightarrow 0$ we arrive at an $L^{1}$-dissipative solver for (4.2), defined for all $u_{0} \in L^{\infty}$. By Remark 3.29, this implicitly defined "vanishing viscosity solver" is characterized by some complete maximal $L^{1} D$ germ that we now want to determine. Its dual germ $\mathcal{G}^{*}$ consists of all elementary solutions (1.10) that are trajectories of this solver. According to GELFAnd [41] (see [27,44]), for all $\left(u^{l}, u^{r}\right)$ satisfying

$$
\mid \begin{aligned}
& f\left(u^{l}\right)=f\left(u^{r}\right) \text { and either } u^{l}=u^{r}, \text { or } \\
& \operatorname{sign}\left(u^{r}-u^{l}\right)\left(f(c)-f\left(u^{l}\right)\right)>0 \text { for all } c \text { between } u^{l} \text { and } u^{r},
\end{aligned}
$$

there exist standing-wave profiles $W: \mathbb{R} \rightarrow \mathbb{R}$ such that $\lim _{\xi \rightarrow-\infty} W(\xi)=u^{l}$, $\lim _{\xi \rightarrow \infty} W(\xi)=u^{r}$, and $W\left(\frac{x}{\varepsilon}\right)$ solves (4.3) for all $\varepsilon>0$. Thus solutions of the form (1.10) with $\left(u^{l}, u^{r}\right)$ as in (4.4) are explicit limits, as $\varepsilon \rightarrow 0$, of solutions of (4.3). Therefore these solutions are trajectories of the vanishing viscosity solver, 
and we can define the "Gelfand" germ $\mathcal{G}_{G}$ as the set of all pairs $\left(u^{l}, u^{r}\right)$ satisfying (4.4); $\mathcal{G}_{G}$ is $L^{1} D$ because the vanishing viscosity solver is $L^{1}$ dissipative by construction.

Let us look at the closure $\overline{\mathcal{G}_{G}}$ of $\mathcal{G}_{G}$. One checks that $\overline{\mathcal{G}_{G}} \supset \mathcal{G}^{\prime}$, where $\mathcal{G}^{\prime}$ is the set of all pairs $\left(u^{l}, u^{r}\right)$ such that (4.4) is satisfied with the inequality " $\geqq 0$ " instead of "> 0". But $\mathcal{G}^{\prime}$ is known to be complete (see [27,41,44]), therefore $\overline{\mathcal{G}_{G}}$ is a complete germ. By Proposition $4.5\left(\right.$ ii), $\mathcal{G}_{G}$ is a definite germ with complete closure; more exactly, $\mathcal{G}_{G}^{*}=\overline{\mathcal{G}_{G}}=\mathcal{G}^{\prime}$, because $\mathcal{G}^{\prime}$ turns out to be an $L^{1} D$ germ. Therefore we can identify the "vanishing viscosity" germ with the unique maximal $L^{1} D$ extension of $\mathcal{G}_{G}$, that is, with its closure $\overline{\mathcal{G}_{G}}$.

Now $\mathcal{G}_{G^{-}}$and $\mathcal{G}_{V K r}$-entropy solutions must coincide, because $\mathcal{G}_{V K r} \subset \mathcal{G}_{G}$ (see their definitions) and $\mathcal{G}_{V K r}$ was shown to be definite; in addition, we infer that $\mathcal{G}_{V K r}^{*}$ coincides with $\overline{\mathcal{G}_{G}}$. In conclusion, the germs $\mathcal{G}_{V K r}$ and $\mathcal{G}_{G}$ have different motivation, but they both lead to the classical Kruzhkov entropy solutions.

Remark 4.8. In closing, we mention that non-classical $L^{1}$-dissipative solvers exist for (4.2). For example, for the Hopf-Burgers flux $f(u)=\frac{u^{2}}{2}, \overline{\mathcal{G}_{G}} \neq \mathbb{R} \times \mathbb{R}$. Take any pair $\left(u^{l}, u^{r}\right) \notin \overline{\mathcal{G}_{G}}$, which satisfies the Rankine-Hugoniot condition, and then set $\mathcal{G}^{\prime \prime}=\left\{\left(u^{l}, u^{r}\right)\right\}$. Take an arbitrary maximal $L^{1} D$-extension $\mathcal{G}^{\prime \prime \prime}$ of $\mathcal{G}^{\prime \prime}$; the associated $\mathcal{G}^{\prime \prime \prime}$-entropy solutions are, in general, different from the Kruzhkov entropy solutions! Notice that since $\left(u^{l}, u^{r}\right) \notin \overline{\mathcal{G}_{G}}=\mathcal{G}_{V K r}^{*}$, some of the constant elementary solutions in $\mathcal{G}_{V K r}$ will not be trajectories for a non-classical solver. Finally, we recall that physically motivated - but not $L^{1}$-dissipative-non-classical solvers for, say, the nonconvex conservation law $u_{t}+\left(u^{3}\right)_{x}=0$ have been extensively studied in the recent years, see the book of LEFLOCH [58].

\subsection{Increasing surjective fluxes $f^{l, r}$}

BAITI and JENSSEN [15] have proposed an entropy formulation for (1.7) with strictly increasing and uniformly Lipschitz continuous fluxes $f^{l}, f^{r}$ (strictly decreasing functions can be treated similarly).

In what follows, we assume that there is a set $W \subset \mathbb{R}$ such that $f^{l, r}: U \mapsto W$ are non-decreasing and onto. The corresponding germ $\mathcal{G}_{R H}$ consists of all pairs $\left(u^{l}, u^{r}\right) \in U \times U$ that satisfy the Rankine-Hugoniot condition (1.9).

Due to the monotonicity of $f^{l, r},(1.11)$ holds for all $\left(u^{l}, u^{r}\right),\left(\hat{u}^{l}, \hat{u}^{r}\right) \in \mathcal{G}_{R H}$. Thus $\mathcal{G}_{R H}$ is an $L^{1} D$ germ; it is a maximal $L^{1} D$ germ because $\mathcal{G}_{R H}$ admits no nontrivial extension. Finally, $\mathcal{G}_{R H}$ is complete. Indeed, taking $\left(u_{-}, u_{+}\right) \in U \times U$, there exists $u^{r} \in U$ such that $\left(u_{-}, u^{r}\right) \in \mathcal{G}_{R H}$; take any $u^{r} \in\left(f^{r}\right)^{-1}\left(f^{l}\left(u_{-}\right)\right)$. Furthermore, the graph $\theta^{r}\left(\cdot, u^{r}\right)$ coincides with the graph of $f^{r}$. Therefore $u_{+} \in$ Dom $\theta^{r}\left(\cdot, u^{r}\right)$, and the Riemann problem (1.7), (1.8) admits a weak solution of the form (RPb-sol.) with the intermediate states $\left(u_{-}, u^{r}\right) \in \mathcal{G}_{R H}$. Definition 3.15 of $\mathcal{G}_{R H}$-entropy solutions then turns into the definition of an admissible solution in the sense of BAITI and JENSSEN [15], adapted to the model equation (1.7).

In passing, notice that a simpler equivalent choice of the admissibility germ is possible. Indeed, we can take $\mathcal{G}_{\emptyset}=\emptyset$. It possesses a unique maximal $L^{1} D$ 
extension, namely $\left(\mathcal{G}_{\emptyset}\right)^{*}=\mathcal{G}_{R H}$. Thus $\mathcal{G}_{\emptyset}$ is a definite germ; by Remark 3.12 , the $\mathcal{G}_{R H^{-}}$and $\mathcal{G}_{\emptyset}$-entropy solutions coincide.

The entropy dissipation property (3.8) of a $\mathcal{G}_{\emptyset}$-entropy solution $u$ imposes no restriction on the jump of $u$ across the flux discontinuity, so the only restriction is the Rankine-Hugoniot condition: $\gamma^{l} f^{l}(u)=\gamma^{r} f^{r}(u)$ (which is equivalent to $\left(\gamma^{l} V^{l} u, \gamma^{r} V^{r} u\right) \in V \mathcal{G}_{R H}=V\left(\mathcal{G}_{\emptyset}\right)^{*}$ of Definition 3.8(ii), since $f^{l, r}$ are monotone).

\subsection{Other cases with monotone fluxes $f^{l, r}$}

We have two different situations.

If we assume that on $U \subset \mathbb{R}, f^{l}$ is non-decreasing and $f^{r}$ is non-increasing, then the germ $\mathcal{G}_{R H}$ of Section 4.4 is still a maximal $L^{1} D$ germ, but it is not complete. Indeed, for all $u^{l, r} \in U$ the graphs $\theta^{l, r}\left(\cdot, u^{l, r}\right)$ are constant; they are the sections of the graphs of $f^{l, r}$ at the levels $f^{l, r}\left(u^{l, r}\right)$, respectively. Therefore, unless the Riemann data $\left(u_{-}, u_{+}\right)$belong to $\mathcal{G}_{R H}$, there exist no solutions to (1.7), (1.8) under the form (RPb-sol.) with $\left(u^{l}, u^{r}\right) \in \mathcal{G}_{R H}$.

Next, consider the case where $f^{l}$ is non-decreasing and $f^{r}$ is non-increasing, on $U \subset \mathbb{R}$. If $f^{l}(U) \cap f^{r}(U)=\emptyset$, then the Rankine-Hugoniot condition (1.9) never holds, that is, there exist no weak solutions of (1.7).

Therefore, let us assume in addition that the ranges of $f^{l}$ and $f^{r}$ are not disjoint. We claim that in this case, for any pair $(A, B)$ such that the Rankine-Hugoniot condition $f^{l}(A)=f^{r}(B)$ holds, the germ $\mathcal{G}_{(A, B)}=\{(A, B)\}$ is a definite complete germ. The corresponding maximal $L^{1} D$ extension of $\mathcal{G}_{(A, B)}$ is its closure $\overline{\mathcal{G}}_{(A, B)}$. Indeed, it is evident that $\overline{\mathcal{G}}_{(A, B)}$ is an $L^{1} D$ germ. If $\mathcal{G}$ is a maximal $L^{1} D$ extension of $\mathcal{G}_{(A, B)}$, then $(A, B) \in \mathcal{G}$. Notice that the graph $\theta^{l}(\cdot, A)$ coincides with the graph of $f^{l}$, and the graph $\theta^{r}(\cdot, B)$ coincides with the graph of $f^{r}$. In particular, both graphs have $U$ for their domain. It follows by Propositions 4.6 and 4.4(iii) that for all $\left(u_{-}, u_{+}\right) \in U \times U$, either $\left(u_{-}, u_{+}\right) \notin \overline{\mathcal{G}}=\mathcal{G}$ or $\left(u_{-}, u_{+}\right) \in \overline{\mathcal{G}}_{(A, B)}$. Thus $\mathcal{G}=\overline{\mathcal{G}}_{(A, B)}$. Finally, for all $\left(u_{-}, u_{+}\right) \in U \times U$, the Riemann problem (1.7), (1.8) admits a weak solution of the form (RPb-sol.) with $\left(u^{l}, u^{r}\right)=(A, B)$.

Notice that the germs $\mathcal{G}_{(A, B)}, \mathcal{G}_{\left(A^{\prime}, B^{\prime}\right)}$ described above have different maximal $L^{1} D$ extensions if and only if $f^{l}(A)=f^{r}(B) \neq f^{l}\left(A^{\prime}\right)=f^{r}\left(B^{\prime}\right)$. Hence, whenever $f^{l}(U) \cap f^{r}(U)$ contains more then one point, there exist infinitely many two by two non-equivalent definitions of $\mathcal{G}$-entropy solutions, each definition being based on a complete definite admissibility germ.

\subsection{The Audusse-Perthame adapted entropies}

Now we consider the case of two fluxes $f^{l, r}$ such that for some $u_{o}^{l, r} \in \mathbb{R}, f^{l, r}$ : $\left(-\infty, u_{o}^{l, r}\right] \mapsto \mathbb{R}_{+}$are bijective decreasing functions and $f^{l, r}:\left[u_{o}^{l, r}, \infty\right) \mapsto \mathbb{R}_{+}$ are bijective increasing functions, see Fig. 1 for an example. This is the context of Audusse and Perthame in [13], reduced to our model case (1.7). The work [13] applies in more generality; it was recently revisited by PANOV [74], who showed that a suitable change of variables reduces the Audusse and Perthame definition 


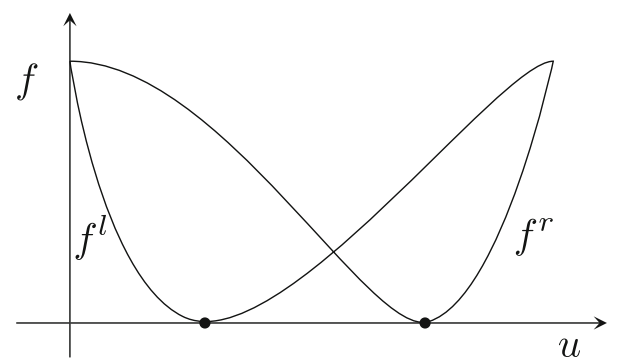

Fig. 1. Typical left and right fluxes covered by the framework of Audusse and Perthame. Bold dots represent $u_{o}^{l, r}$

to the definition of KruzhKov [54]. Here we do not take advantage of Panov's observation.

The notion of entropy solutions in [13] is based on "partially adapted entropies". These are, for our model case, entropies built from a step function $c(x)$ associated with a pair $\left(c^{l}, c^{r}\right) \in \mathcal{G}_{A P}: z \mapsto \eta_{c(x)}(z)=|z-c(x)|$. Here $\mathcal{G}_{A P}$ is the "Audusse-Perthame" germ given by

$$
\left(u^{l}, u^{r}\right) \in \mathcal{G}_{A P} \Longleftrightarrow f^{l}\left(u^{l}\right)=f^{r}\left(u^{r}\right), \operatorname{sign}\left(u^{l}-u_{o}^{l}\right)=\operatorname{sign}\left(u^{r}-u_{o}^{r}\right) .
$$

Definition 3.15 (under the form of Proposition 3.17) of $\mathcal{G}_{A P}$-entropy solutions reduces to the definition of Audusse and Perthame, for which uniqueness is shown in $[13,74]$. Thus it is not surprising that $\mathcal{G}_{A P}$ is an $L^{1} D$ germ; property (1.11) is checked directly from the definition (4.5) of $\mathcal{G}_{A P}$.

Proposition 4.9. The germ $\mathcal{G}_{A P}$ is complete.

Proof. Denote by $h_{-}^{l, r}$ the inverses of $\left.f^{l, r}\right|_{\left(-\infty, u_{o}^{l, r}\right]}$; denote by $h_{+}^{l, r}$ the inverses of $\left.f^{l, r}\right|_{\left[u_{o}^{l, r}, \infty\right)}$. The domains of $\theta^{l, r}\left(\cdot, u^{l, r}\right)$ are easy to calculate:

$$
\begin{aligned}
& \operatorname{Dom} \theta^{l}\left(\cdot, u^{l}\right)= \begin{cases}\left(-\infty, h_{+}^{l}\left(f^{l}\left(u^{l}\right)\right)\right], & \text { if } u^{l} \leqq u_{o}^{l}, \\
\left\{u^{l}\right\}, & \text { if } u^{l}>u_{o}^{l}\end{cases} \\
& \operatorname{Dom} \theta^{r}\left(\cdot, u^{r}\right)= \begin{cases}\left\{u^{r}\right\}, & \text { if } u^{r}<u_{o}^{r}, \\
{\left[h_{-}^{r}\left(f^{r}\left(u^{r}\right)\right), \infty\right),} & \text { if } u^{r} \geqq u_{o}^{r} .\end{cases}
\end{aligned}
$$

According to Remark 4.1, we have to show that $\mathbb{R} \times \mathbb{R}$ is covered by

$$
\bigcup_{\left(u^{l}, u^{r}\right) \in \mathcal{G}_{A P}} \operatorname{Dom} \theta^{l}\left(\cdot, u^{l}\right) \times \operatorname{Dom} \theta^{r}\left(\cdot, u^{r}\right) .
$$

Indeed, take $\left(u_{-}, u_{+}\right) \in \mathbb{R} \times \mathbb{R}$. We have five cases:

- if $u_{-} \leqq u_{o}^{l}, u_{+} \geqq u_{o}^{r}$, then $\left(u_{-}, u_{+}\right) \in \operatorname{Dom} \theta^{l}\left(\cdot, u_{o}^{l}\right) \times \operatorname{Dom} \theta^{r}\left(\cdot, u_{o}^{r}\right)$, and $\left(u_{o}^{l}, u_{o}^{r}\right) \in \mathcal{G}_{A P}$ (notice that $f^{l, r}\left(u_{o}^{l, r}\right)=0$, by the assumptions on $\left.f^{l, r}\right)$;

- $\quad$ in the case $u_{-} \leqq u_{o}^{l}, u_{+}<u_{o}^{r}$, set $s=f^{r}\left(u_{+}\right)$; then the pair $\left(u_{-}, u_{+}\right)$belongs to $\operatorname{Dom} \theta^{l}\left(\cdot, h_{-}^{l}(s)\right) \times \operatorname{Dom} \theta^{r}\left(\cdot, u_{+}\right)$, and $\left(h_{-}^{l}(s), u_{+}\right) \in \mathcal{G}_{A P}$; 
- the same is true if $u_{-}>u_{o}^{l}, u_{+}<u_{o}^{r}$ and $f^{l}\left(u_{-}\right) \leqq f^{r}\left(u_{+}\right)=s$;

- $\quad$ in the case $u_{-}>u_{o}^{l}, u_{+} \geqq u_{o}^{r}$, set $s=f^{l}\left(u_{-}\right)$; then the pair $\left(u_{-}, u_{+}\right)$belongs to $\operatorname{Dom} \theta^{l}\left(\cdot, u_{-}\right) \times \operatorname{Dom} \theta^{r}\left(\cdot, h_{+}^{r}(s)\right)$, and $\left(u_{-}, h_{+}^{r}(s)\right) \in \mathcal{G}_{A P}$;

- the same is true if $u_{-}>u_{o}^{l}, u_{+}<u_{o}^{r}$ and $s=f^{l}\left(u_{-}\right) \geqq f^{r}\left(u_{+}\right)$.

Hence, $\left(u_{-}, u_{+}\right) \in \operatorname{Dom} \theta^{l}\left(\cdot, u^{l}\right) \times \operatorname{Dom} \theta^{r}\left(\cdot, u^{r}\right)$ for some $\left(u^{l}, u^{r}\right) \in \mathcal{G}_{A P}$.

Notice that Propositions 4.9 and 4.5(i) imply that the maximal $L^{1} D$ extension $\left(\mathcal{G}_{A P}\right)^{*}$ of the germ $\mathcal{G}_{A P}$ coincides with its closure $\overline{\mathcal{G}}_{A P}$. The above analysis gives

$$
\begin{aligned}
\overline{\mathcal{G}}_{A P} & =\mathcal{G}_{A P} \cup\left\{\left(u^{l}, u^{r}\right) \mid f^{l}\left(u^{l}\right)=f^{r}\left(u^{r}\right), u^{l}>u_{o}^{l}, u^{r}<u_{o}^{r}\right\} \\
& \equiv\left\{\left(u^{l}, u^{r}\right) \mid f^{l}\left(u^{l}\right)=f^{r}\left(u^{r}\right), \operatorname{sign}^{-}\left(u^{l}-u_{o}^{l}\right) \operatorname{sign}^{+}\left(u^{r}-u_{o}^{r}\right) \leqq 0\right\} .
\end{aligned}
$$

Now, consider the germ $\mathcal{G}_{\left(u_{o}^{l}, u_{o}^{r}\right)}:=\left\{\left(u_{o}^{l}, u_{o}^{r}\right)\right\}$. We find that $\left(\mathcal{G}_{\left(u_{o}^{l}, u_{o}^{r}\right)}\right)^{*}=$ $\overline{\mathcal{G}}_{A P}$, so $\left(\mathcal{G}_{\left(u_{o}^{l}, u_{o}^{r}\right)}\right)^{*}$ is an $L^{1} D$ germ; by Proposition $3.4(\mathrm{v}), \mathcal{G}_{\left(u_{o}^{l}, u_{o}^{r}\right)}$ is a definite germ. By Remark 3.12(ii), $\mathcal{G}_{\left(u_{o}^{l}, u_{o}^{r}\right)}$-entropy solutions of (1.7) coincide with $\mathcal{G}_{A P}$-entropy solutions, thus they coincide with entropy solutions in the sense of Audusse and Perthame [13]. The completeness of $\left(\mathcal{G}_{\left(u_{o}^{l}, u_{o}^{r}\right)}\right)^{*}$ follows from Proposition 4.9. The existence of $\mathcal{G}_{\left(u_{o}^{l}, u_{o}^{r}\right)}$-entropy solutions can be obtained from Theorem 6.4 or from the adapted viscosity method of Theorem 6.3 in Section 6 (see also [74]).

Moreover, different notions of entropy solution can be considered for the same configuration of fluxes. Indeed, take any pair $(A, B) \in\left(-\infty, u_{o}^{l}\right] \times\left[u_{o}^{r}, \infty\right)$ which fulfills the Rankine-Hugoniot condition; set

$$
s_{(A, B)}:=f^{l}(A)=f^{r}(B) .
$$

The germ $\mathcal{G}_{(A, B)}:=\{(A, B)\}$ turns out to be definite, and its unique maximal extension $\left(\mathcal{G}_{(A, B)}\right)^{*}$ is given by

$$
\begin{aligned}
& \left(u^{l}, u^{r}\right) \in\left(G_{(A, B)}\right)^{*} \\
& \Longleftrightarrow f^{l}\left(u^{l}\right)=f^{r}\left(u^{r}\right) \geqq s_{(A, B)}, \operatorname{sign}^{-}\left(u^{l}-A\right) \operatorname{sign}^{+}\left(u^{r}-B\right) \leqq 0 .
\end{aligned}
$$

In the same way as in Proposition 4.9, we check that $\left(\mathcal{G}_{(A, B)}\right){ }^{*}$ is complete. Clearly, the choice $(A, B)=\left(u_{o}^{l}, u_{o}^{r}\right)$ discussed above is a particular case. By analogy with the case considered by Adimurthi et AL. [4] (see also [22] and Section 4.8), such pairs $(A, B)$ will be called $(A, B)$-connections.

We easily check that (4.6) accounts for all maximal $L^{1} D$ germs, for the fluxes considered in this paragraph. The monotonicity condition on $f^{l, r}$ can be relaxed, allowing for non-strictly monotone on $\left(-\infty, u_{o}^{l, r}\right]$ and on $\left[u_{o}^{l, r}, \infty\right)$ fluxes $f^{l, r}$.

We see that the definition of entropy solutions due to Audusse and Perthame [13], as applied to the model case (1.7), corresponds to one among infinitely many choices of $L^{1}$-contractive semigroups of solutions. Each semigroup is most concisely determined by a choice of an $(A, B)$-connection, that is, by a singleton $\{(A, B)\}$ from the set $\left(-\infty, u_{o}^{l}\right] \times\left[u_{o}^{r}, \infty\right)$; any such singleton is a definite germ with complete dual. 

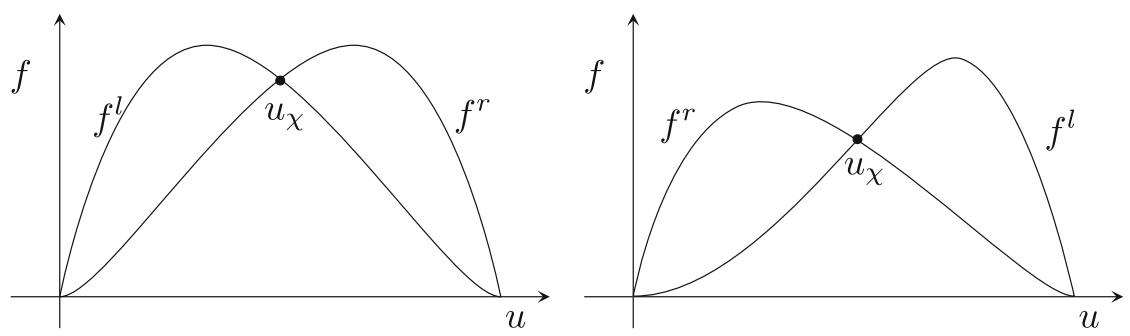

Fig. 2. Left crossing condition (4.8) satisfied. Right crossing condition violated

\subsection{The Karlsen-Risebro-Towers entropy condition}

In $[49,52,80,81]$, the definition of an entropy solution is similar to Definition 3.15 , with (3.8) replaced by the inequality

$$
\begin{aligned}
& \int_{\mathbb{R}_{+}} \int_{\mathbb{R}}\left\{|u-c| \xi_{t}+\mathfrak{q}(x, u, c) \xi_{x}\right\} \\
& \quad-\int_{\mathbb{R}}\left|u_{0}-c\right| \xi(0, x)+\int_{\mathbb{R}_{+}}\left|f^{r}(c)-f^{l}(c)\right| \xi(t, 0) \geqq 0,
\end{aligned}
$$

that should hold for all $c \in \mathbb{R}$ and for all $\xi \in \mathcal{D}([0, \infty) \times \mathbb{R}), \xi \geqq 0$. Uniqueness for (1.7) under this admissibility condition is shown for fluxes $f^{\bar{l}, r}$ satisfying the so-called crossing condition, see Fig. 2:

$$
\exists u_{\chi} \in \overline{\mathbb{R}} \operatorname{such} \text { that } \operatorname{sign}\left(z-u_{\chi}\right)\left(f^{r}(z)-f^{l}(z)\right) \geqq 0 \forall z \in U .
$$

Let us analyze the underlying germ. For the sake of simplicity, assume that $f^{l, r}$ are non-constant on non-degenerate intervals. Then the singular mappings $V^{l, r}$ are strictly monotone. In this case, for all $u$ satisfying (4.7) there exist strong traces $t \mapsto\left(\gamma^{l, r} u\right)(t)$; in addition, the Rankine-Hugoniot condition holds for these left and right traces. As in the proof of Theorem 3.18, for $h>0$ and $\xi \in \mathcal{D}([0, \infty) \times \mathbb{R})$, $\xi \geqq 0$, we take $\psi_{h}=\frac{(h-|x|)^{+}}{h} \xi$ as the test function in (4.7) and send $h \rightarrow 0$. The final result reads

$$
\int_{\mathbb{R}_{+}}\left(q^{l}\left(\left(\gamma^{l} u\right)(t), c\right)-q^{r}\left(\left(\gamma^{r} u\right)(t), c\right)+\left|f^{r}(c)-f^{l}(c)\right|\right) \xi(t, 0) \geqq 0 .
$$

Setting $u^{l, r}(t):=\left(\gamma^{l, r} u\right)(t)$, the inequalities (4.9) yield the condition

$$
\begin{aligned}
& \forall c \in \mathbb{R}, \quad q^{l}\left(\left(u^{l}\right)(t), c\right)-q^{r}\left(\left(u^{r}\right)(t), c\right)+\left|f^{r}(c)-f^{l}(c)\right| \geqq 0, \\
& \quad \text { for almost every } t>0 .
\end{aligned}
$$

A case study shows that (4.10) defines the following germ:

$$
\begin{aligned}
& \left(u^{l}, u^{r}\right) \in \mathcal{G}_{K R T} \text { if and only if } f^{l}\left(u^{l}\right)=f^{r}\left(u^{r}\right)=: s \\
& \text { and }\left\{\begin{array}{lll}
\text { either } u^{l}=u^{r} ; \\
\text { or } u^{l}<u^{r} \text { and } \forall z \in\left[u^{l}, u^{r}\right], & \max \left\{f^{l}(z), f^{r}(z)\right\} \geqq s ; \\
\text { or } u^{l}>u^{r} \text { and } \forall z \in\left[u^{r}, u^{l}\right], & \min \left\{f^{l}(z), f^{r}(z)\right\} \leqq s .
\end{array}\right.
\end{aligned}
$$


This definition of $\mathcal{G}_{K R T}$ is valid also if $f^{l, r}$ are allowed to degenerate (become constant on intervals). The germ $\mathcal{G}_{K R T}$ is closed.

Proceeding as in the proof of Theorem 3.18, we have

Remark 4.10. A function $u$ is an entropy solution of (1.7) in the sense of [52] if and only if it is a solution in the sense of Definition 3.8 with $\mathcal{G}^{*}$ replaced by $\mathcal{G}_{K} R T$. Thus, any function of the form (RPb-sol.) with intermediate states $\left(u^{l}, u^{r}\right) \in \mathcal{G}_{K R T}$ is a solution of the Riemann problem (1.7), (1.8) in the sense of [52].

Let us show that the crossing condition (4.8) is sufficient for the germ $\mathcal{G}_{K R T}$ to be $L^{1} D$ and, moreover, maximal $L^{1} D$.

\section{Proposition 4.11.}

(i) The germ $\mathcal{G}_{K R T}$ admits no non-trivial $L^{1} D$ extension.

(ii) If the fluxes $f^{l, r}$ satisfy the crossing condition (4.8), then the germ $\mathcal{G}_{K R T}$ is $L^{1} D$ (and therefore it is maximal $L^{1} D$ ).

Proof. (i) Assume that there exists $\left(\hat{u}^{l}, \hat{u}^{r}\right) \notin \mathcal{G}_{K R T}$ such that $\mathcal{G}_{K R T} \cup\left\{\left(\hat{u}^{l}, \hat{u}^{r}\right)\right\}$ is $L^{1} D$. We can assume that $\hat{u}^{l}<\hat{u}^{r}$ and that there exists $z \in\left(\hat{u}^{l}, \hat{u}^{r}\right)$ such that both $f^{l}(z)$ and $f^{r}(z)$ are strictly smaller than $\hat{s}:=f^{l, r}\left(\hat{u}^{l, r}\right)$ (the other case is symmetric). We claim the

$$
\exists\left(u^{l}, u^{r}\right) \in \mathcal{G}_{K R T} \text { such that } \hat{u}^{l}<u^{l} \leqq u^{r}<\hat{u}^{r} \text { and } f^{l, r}\left(u^{l, r}\right)<\hat{s} .
$$

Indeed, we can assume that $f^{l}(z) \leqq f^{r}(z)<\hat{s}$ (the other case is symmetric). We set $\bar{s}:=f^{r}(z)$ and examine the three possibilities.

- Either $f^{l}, f^{r}$ have a crossing point $u_{\chi} \in\left[\hat{u}^{l}, z\right]$ such that $f^{l, r}\left(u_{\chi}\right) \leqq \bar{s}$. Then it is clear that $\hat{u}^{l}<u_{\chi} \leqq z<\hat{u}^{r}$, so that $u^{l, r}:=u_{\chi}$ satisfies (4.12).

- Or $f^{r}>f^{l}$ on $\left[\hat{u}^{l}, z\right]$. Set $u^{l, r}:=\inf \left\{\zeta \in\left[\hat{u}^{l}, z\right] \mid f^{l, r}(\zeta)=\bar{s}\right\}$. Since $f^{r}(z)=$ $\bar{s}, u^{r}$ is well defined and $u^{r} \leqq z<\hat{u}^{r}$. Since $f^{l}\left(u^{l}\right)=\hat{s}>\bar{s} \geqq f^{l}(z), u^{l}$ is well defined and $\hat{u}^{l}<u^{l}$. Because $f^{r}>f^{l}$ and by the choice of $u^{l, r}$, we have $u^{l}<u^{r}, f^{r}(\zeta) \geqq \bar{s}=f^{l, r}\left(u^{l, r}\right) \forall \zeta \in\left[u^{l}, u^{r}\right]$. Thus $\left(u^{l}, u^{r}\right) \in \mathcal{G}_{K R T}$ and (4.12) holds.

- Or else, $f^{l, r}$ have a crossing point $u_{\chi} \in\left[\hat{u}^{l}, z\right]$ with $f^{l, r}\left(u_{\chi}\right)>\bar{s}$. Then we choose the closest to $z$ crossing point $u_{\chi}$ with the above property, and set $u^{l, r}:=\left\{\zeta \in\left[u_{\chi}, z\right] \mid f^{l, r}(\zeta)=\bar{s}\right\}$. As in the previous case, we check that $u^{l, r}$ are well defined, moreover, $u^{l}<u^{r}$ and $f^{r}(\zeta) \geqq \bar{s}=f^{l, r}\left(u^{l, r}\right)$ for all $\zeta \in\left[u^{l}, u^{r}\right]$. Thus, $\left(u^{l}, u^{r}\right) \in \mathcal{G}_{K R T}$ and (4.12) holds.

Now consider the pair $\left(\hat{u}^{l}, \hat{u}^{r}\right)$, and the pair $\left(u^{l}, u^{r}\right)$ which satisfies (4.12). They violate (1.11); thus $\mathcal{G}_{K R T} \cup\left\{\left(\hat{u}^{l}, \hat{u}^{r}\right)\right\}$ is not $L^{1} D$. This contradiction shows that $\mathcal{G}_{K R T}$ has no non-trivial $L^{1} D$ extension.

(ii) Let $\left(u^{l}, u^{r}\right),\left(\hat{u}^{l}, \hat{u}^{r}\right)$ be two pairs satisfying the Rankine-Hugoniot condition; set $s:=f^{l, r}\left(u^{l, r}\right), \hat{s}:=f^{l, r}\left(\hat{u}^{l, r}\right)$. Upon exchanging the roles of $u^{l, r}$ and $\hat{u}^{l, r}$, we easily find that (1.11) fails if and only if we have simultaneously $s<\hat{s}, u^{l}>\hat{u}^{l}$, and $u^{r}<\hat{u}^{r}$. These conditions are realized if and only if $s<\hat{s}$ and

$$
\begin{gathered}
\text { either } \hat{u}^{l}<u^{l} \leqq u^{r}<\hat{u}^{r} \text {, or } \hat{u}^{l} \leqq u^{r} \leqq u^{l} \leqq \hat{u}^{r} \text {, or } u^{r} \leqq \hat{u}^{l}<u^{l} \leqq \hat{u}^{r}, \\
\text { or } \hat{u}^{l} \leqq u^{r}<\hat{u}^{r} \leqq u^{l} \text {, or } u^{r} \leqq \hat{u}^{l} \leqq \hat{u}^{r} \leqq u^{l} \text {, or } u^{r}<\hat{u}^{r} \leqq \hat{u}^{l}<u^{l}
\end{gathered}
$$


We check that in all these cases except for the first one and the last one (4.11) never holds simultaneously for $\left(u^{l}, u^{r}\right)$ and $\left(\hat{u}^{l}, \hat{u}^{r}\right)$. In the two cases that were excepted (4.11) can hold simultaneously for $\left(u^{l}, u^{r}\right),\left(\hat{u}^{l}, \hat{u}^{r}\right)$ only if the crossing condition (4.8) fails.

This shows that if (4.8) holds, then (1.11) holds for all $\left(u^{l}, u^{r}\right),\left(\hat{u}^{l}, \hat{u}^{r}\right) \in$ $\mathcal{G}_{K R T}$. We conclude that $\mathcal{G}_{K R T}$ is an $L^{1} D$ germ; by (i), it is maximal $L^{1} D$.

Now let us look at one case where the crossing condition (4.8) fails. Consider the fluxes in Section 4.6 (that is, of the type in Fig. 1) with $u_{o}^{l}<u_{o}^{r}$. We claim that $\mathcal{G}_{K R T}$ is not an $L^{1} D$ germ. Indeed, with the notation of Section 4.6, let

$$
\delta=\inf \left\{s \mid \exists u \in\left(u_{o}^{l}, u_{o}^{r}\right) \text { such that } f^{l}(u)=f^{r}(u)=s\right\} ;
$$

then for all $s \in(0, \delta]$, the pair $\left(u^{l}, u^{r}\right):=\left(h_{-}^{l}(s), h_{+}^{r}(s)\right)$ belongs to $\mathcal{G}_{K R T}$. Also $\left(\hat{u}^{l}, \hat{u}^{r}\right):=\left(u_{o}^{l}, u_{o}^{r}\right) \in \mathcal{G}_{K R T}$, but then (1.11) is violated. Another way to prove that $\mathcal{G}_{K R T}$ is not $L^{1} D$ is to notice that the definite germ $\mathcal{G}_{\left(u_{o}^{l}, u_{o}^{r}\right)}=\left\{\left(u_{o}^{l}, u_{o}^{r}\right)\right\}$ is contained within $\mathcal{G}_{K R T}$, but, according to (4.6), $\left(u^{l}, u^{r}\right)$ does not belong to the unique maximal $L^{1} D$ extension $\left(\mathcal{G}_{\left(u_{o}^{l}, u_{o}^{r}\right)}\right)^{*}$ of $\mathcal{G}_{\left(u_{o}^{l}, u_{o}^{r}\right)}$.

Based on this fact, we can construct a non-uniqueness example for one simple case where the crossing condition (4.8) fails.

Example 4.12. Consider the fluxes $f^{l, r}: z \mapsto\left|z-u_{o}^{l, r}\right|$ with $u_{o}^{l}<u_{o}^{r}$. Then the entropy condition of [52] is not sufficient to single out a unique solution to the Riemann problem (1.7), (1.8) with $\left(u_{-}, u_{+}\right)=\left(u_{0}^{l}-s, u_{0}^{r}+s\right)$, for all $s$ in $\left(0, \frac{1}{2}\left(u_{o}^{r}-u_{o}^{l}\right)\right]$. Indeed, the result follows by the above analysis and by Remark 4.10. We have $\left(u_{-}, u_{+}\right) \in \mathcal{G}_{K R T}$; thus the function $u(t, x)=$ $u_{-} \mathbb{1}_{\{x<0\}}+u_{+} \mathbb{1}_{\{x>0\}}$ is one solution. Because $u^{l, r} \in \operatorname{Dom} \theta^{l, r}\left(\cdot, u_{o}^{l, r}\right)$, there exists another solution of the form (RPb-sol.) with intermediate states $\left(u_{o}^{l}, u_{o}^{r}\right) \in \mathcal{G}_{K R T}$.

In contrast, if we consider the fluxes as in Section 4.6 with $u_{o}^{l}>u_{o}^{r}$ such that the crossing condition (4.8) holds, then there exists a unique crossing point $u_{\chi}$ of $f^{l}$ and $f^{r}$, and $u_{\chi} \in\left(u_{o}^{r}, u_{o}^{l}\right)$. In this case, one easily checks that $\mathcal{G}_{K R T}$ coincides with the complete maximal $L^{1} D$ germ $\left(\left(\mathcal{G}_{\left(u_{\chi}, u_{\chi}\right)}\right)^{*}\right.$. Another very similar example is given in Section 4.8 .

Let us also look at the configuration of fluxes considered in Section 4.4. In this case, irrespective of the crossing condition (4.8), we always have $\mathcal{G}_{K R T}=\mathcal{G}_{R H}$; thus $\mathcal{G}_{K R T}$ is a complete maximal $L^{1} D$ germ. Also in the case $f^{l} \equiv f^{r}, \mathcal{G}_{K R T}$ is a complete maximal $L^{1} D$ germ, because it coincides with the Vol'pert-Kruzhkov germ $\mathcal{G}_{V K r}$ (here, the crossing condition is satisfied).

Finally, we point out that the germ $\mathcal{G}_{K R T}$ is closely related to the "vanishing viscosity" germ $\mathcal{G}_{V V}$ which we consider in Section 5; more precisely, we prove there that $\mathcal{G}_{V V}=\mathcal{G}_{K R T}$ whenever the crossing condition (4.8) holds.

In conclusion, the Definition (4.7) of an entropy solution given in [52] can be equivalently reformulated in terms of the germ $\mathcal{G}_{K R T}$ (see Remark 4.10). Whenever the crossing condition (4.8) holds, the germ $\mathcal{G}_{K R T}$ is maximal $L^{1} D$ (see Proposition 4.11) and the unique entropy solution in the sense (4.7) coincides with the unique $\mathcal{G}_{K R T}$-entropy solution (see [52] or Theorem 3.11 for the uniqueness proof). 
For general fluxes, uniqueness may fail, as in Example 4.12; nevertheless, the crossing condition (4.8) is not necessary for the uniqueness of $\mathcal{G}_{K R T}$-entropy solutions.

\subsection{Bell-shaped fluxes}

Many works have been devoted to the case of bell-shaped fluxes. To fix the ideas,

let $U=[0,1]$; assume $f^{l, r}: U \mapsto \mathbb{R}_{+}$are such that $f^{l, r}(0)=0=f^{l, r}(1)$, $f^{l, r}$ are non-decreasing on $\left[0, u_{o}^{l, r}\right]$ and non-increasing on $\left[u_{o}^{l, r}, 1\right]$,

the graphs of $f^{l}, f^{r}$ have at most one crossing point with abscissa in $(0,1)$, and if there is a crossing point $u_{\chi}$, then it lies between the points $u_{o}^{l}, u_{o}^{r}$.

We refer to [22, Figure 1.1] (see also Fig. 2 in the present paper) for a catalogue of different configurations. More general cases with multiple crossing points and oscillating fluxes are considered in [63].

Here the situation is quite similar to the one described in Section 4.6 (we just have to take into account the fact that $u_{o}^{l, r}$ are now points of maxima (not minima) for $f^{l, r}$. There exist infinitely many complete maximal $L^{1} D$ germs. Each of these germs contains one and only one pair $(A, B) \in\left[u_{o}^{l}, 1\right] \times\left[0, u_{o}^{r}\right]$ (see Proposition 4.6). The germ is thus uniquely determined by a choice of the connection $(A, B)$ (see Adimurthi ET AL. [4] and Bürger ET AL. [22]); each maximal $L^{1} D$ germ is the dual $\left(\mathcal{G}_{(A, B)}\right)^{*}$ of the definite germ

$$
\mathcal{G}_{(A, B)}:=\left\{(A, B): A \in\left[u_{o}^{l}, 1\right], B \in\left[0, u_{o}^{r}\right] \text {, and } f^{l}(A)=f^{r}(B)=: s_{(A, B)} \cdot\right\}
$$

We have

$$
\begin{aligned}
& \left(u^{l}, u^{r}\right) \in\left(\mathcal{G}_{(A, B)}\right)^{*} \Longleftrightarrow \\
& f^{l}\left(u^{l}\right)=f^{r}\left(u^{r}\right) \leqq s_{(A, B)}, \operatorname{sign}^{+}\left(u^{l}-A\right) \operatorname{sign}^{-}\left(u^{r}-B\right) \leqq 0 .
\end{aligned}
$$

As in Proposition 4.9, one shows that $\left(G_{(A, B)}\right)^{*}$ is complete. Another way is to use Proposition 3.23 together with the existence result of [22]. Indeed, in view of Proposition 3.17, it is clear that Definition 3.15 of $G_{(A, B)}$-entropy solution is equivalent to the definition given by BüRGER ET AL. [22], which requires that inequality (3.8) holds

- for all $\xi \in \mathcal{D}([0, \infty) \times \mathbb{R}), \xi \geqq 0$, such that $\xi(0, t)=0$; with $\left(c^{l}, c^{r}\right)$ arbitrary; - for all $\xi \in \mathcal{D}([0, \infty) \times \mathbb{R}), \xi \geqq 0$, and $\left(c^{l}, c^{r}\right)=(A, B)$.

The technical genuine nonlinearity restriction on $f^{l, r}$ imposed in [22] can be bypassed. ${ }^{5}$ In Section 6, we give alternative proofs of existence of $\mathcal{G}_{(A, B)}$-entropy solutions, using an adapted viscosity approach and a specific numerical scheme.

5 In works on conservation laws with interface coupling, such as the discontinuous flux problems, there are two reasons to ask for the genuine nonlinearity of the fluxes. Firstly, this assumption simplifies the manipulation of the interface fluxes (see Remarks 2.2, 2.3, where these restrictions are bypassed). Secondly, this assumption yields strong compactness properties (see [60] and [70,73]). In [22], the compactness is achieved with the help of local $B V$ estimates; thus the nonlinearity assumption on $f^{l, r}$ can be dropped. 
Let us point out three specific choices of a connection $(A, B)$. The first one is $(A, B)=(1,0)$, which corresponds to the extremal choice $s_{(A, B)}=0$. Furthermore, we have that $\mathcal{G}_{(1,0)}=\{(1,0),(0,0),(1,1)\}$ (this example is mentioned, for instance by PANOv [74]). The other extremal choice is to pick $(A, B)$ corresponding to the maximum of $s_{(A, B)}$; in this case, we have either $A=u_{o}^{l}$ (whenever $\left.f^{l}\left(u_{o}^{l}\right) \leqq f^{r}\left(u_{o}^{r}\right)\right)$ or $B=u_{o}^{r}$ (whenever $\left.f^{l}\left(u_{o}^{l}\right) \geqq f^{r}\left(u_{o}^{r}\right)\right){ }^{6}$ This is the germ identified by KaAsSchietter [48], Ostrov [66], and Adimurthi and VeERAPPA GowDA [2]; the corresponding solutions (which are $\mathcal{G}_{(A, B)}$-entropy solutions in our vocabulary) have been constructed as limits of various physically relevant regularizations. In the case where $f^{l}$ and $f^{r}$ have no crossing point with abscissa in $(0,1)$, the definition of BACHMANN and VovelLE [14] also leads to this germ, as well as the one of KARLSEN ET AL. [52] (see Section 4.7).

Finally, consider the case where the crossing condition (4.8) holds with $u_{\chi} \in$ $(0,1)$. Then $\left(u_{\chi}, u_{\chi}\right) \in\left[u_{o}^{l}, 1\right] \times\left[0, u_{o}^{r}\right]$. The germ $\mathcal{G}_{\left(u_{\chi}, u_{\chi}\right)}$ has a unique maximal $L^{1} D$ extension $\left(\mathcal{G}_{\left(u_{\chi}, u_{\chi}\right)}\right)^{*}$ which coincides with the germ $\mathcal{G}_{K R T}$ described in Section 4.7. As it was demonstrated by AdIMURTHI ET AL. [4], this germ also comes out of the vanishing viscosity approaches of GIMSE and RISEBRo [42,43] and DIEHL [34-36]. Section 5 below is devoted to a detailed study of the "vanishing viscosity" $\operatorname{germ}(\mathrm{s})$.

\subsection{The case $f^{l} \equiv f^{r}$ and the Colombo-Goatin germs}

Colombo and GoAtin [31] consider the conservation law of the form (1.7) with $f^{l}=f=f^{r}$, which is a bell-shaped function in the sense of Section 4.8; for some $F \in[0, \max f]$, the restriction on the value of the flux, $|f(u(t, 0))| \leqq F$, is imposed. Starting from a regularization of the constraint and using the entropy formulation of KARLSEN ET AL. [52], the authors obtain a definition of entropy solution which leads to well-posedness in the $B V$ framework.

Analyzing the definition of [31], we easily determine the pairs $\left(u^{l}, u^{r}\right)$ for which the elementary solution (1.10) is an entropy solution in the sense of [31]. The set of all these pairs is, in fact, a maximal $L^{1} D$ germ; moreover, this germ is the unique maximal extension $\left(\mathcal{G}_{C G}^{F}\right)^{*}$ of the definite "Colombo-Goatin" germ $\mathcal{G}_{C G}^{F}$ :

$$
\mathcal{G}_{C G}^{F}=\{(A, B): A, B \text { are determined by } A \leqq B, f(A)=f(B)=F\} .
$$

This can be seen as an $(A, B)$-connection of [22] and Section 4.8, except that we are in the particular "degenerate" situation with $f^{l} \equiv f^{r}$.

In fact, in [31] the constraint $F$ depends on $t$. This case can be included in our consideration. We refer to Remark 3.29 and the forthcoming papers [9, 10].

${ }^{6}$ We authorize $f^{l, r}$ to be constant on non-degenerate intervals, therefore $u_{o}^{l, r}$ are not uniquely defined. But all the germs $\mathcal{G}_{\left(u_{o}^{l}, B\right)}\left(\right.$ or $\left.\mathcal{G}_{\left(A, u_{o}^{r}\right)}\right)$ corresponding to different choices of $u_{o}^{l, r}$ have the same closure. 


\section{The vanishing viscosity germ}

There are many possibilities for introducing a vanishing viscosity regularization of (1.7). Gimse and Risebro [42,43] recast (1.7) as a $2 \times 2$ system and add the simplest viscosity terms to both equations. DiEHL [34-36] introduces both the viscosity $\varepsilon u_{x x}$ and a smoothing $\mathfrak{f}^{\delta}$ of the discontinuity in $\mathfrak{f}$; the same approach is pursued in [22]. The regularization term of KAASSCHIETTER [48] is more involved, making appear a capillarity pressure function; the admissibility condition he obtains is different from the ones of [34-36,42,43].

We shall first study the pure vanishing viscosity regularization of (1.7), that is,

$$
u_{t}+\mathfrak{f}(x, u)_{x}=\varepsilon u_{x x}, \quad \mathfrak{f}(x, z)= \begin{cases}f^{l}(z), & x<0 \\ f^{r}(z), & x>0\end{cases}
$$

We pursue the traveling-wave approach of GELFAND [41] (see Section 4.3), which is, in our context, a "standing-wave" approach. Given $f^{l, r}$, define the "standingwave vanishing viscosity" germ by

$$
\left(u^{l}, u^{r}\right) \in \mathcal{G}_{V V}^{s} \Longleftrightarrow\left\{\begin{array}{l}
\text { there exists a function } W: \mathbb{R} \rightarrow \mathbb{R} \text { such that } \\
\lim _{\xi \rightarrow-\infty} W(\xi)=u^{l}, \lim _{\xi \rightarrow \infty} W(\xi)=u^{r}, \text { and } \\
u^{\varepsilon}(t, x)=W(x / \varepsilon) \text { solves }(5.1) \text { in } \mathcal{D}^{\prime}\left(\mathbb{R}_{+} \times \mathbb{R}\right) .
\end{array}\right.
$$

Clearly, if $\left(u^{l}, u^{r}\right) \in \mathcal{G}_{V V}^{s}$, then the elementary stationary solution (1.10) can be obtained as the almost everywhere and $L_{\text {loc }}^{1}$ limit of a sequence of solutions of the regularized equation (5.1).

It turns out that $\mathcal{G}_{V V}^{s}$ can be replaced by a smaller germ which is described explicitly; moreover, we show that $\mathcal{G}_{V V}^{s}$ is definite. The proposition below leads to a definition of the maximal $L^{1} D$ germ $\mathcal{G}_{V V}$, which has already appeared in the work of DIEHL [37].

Proposition 5.1. Consider the germ $\mathcal{G}_{V V}^{o}$ defined as the set of all the pairs $\left(u^{l}, u^{r}\right)$ such that

$$
\begin{aligned}
& f^{l}\left(u^{l}\right)=f^{r}\left(u^{r}\right)=: s, \text { and } \\
& \begin{cases}\text { either } u^{l}=u^{r} ; & \\
\text { or } u^{l}<u^{r} \text { and } & \text { either } f^{l}(z)>s \text { for all } z \in\left(u^{l}, u^{r}\right], \\
& \text { or } f^{r}(z)>s \text { for all } z \in\left[u^{l}, u^{r}\right) ; \\
\text { or } u^{l}>u^{r} \text { and } & \text { either } f^{l}(z)<s \text { for all } z \in\left[u^{r}, u^{l}\right), \\
& \text { or } f^{r}(z)<s \text { for all } z \in\left(u^{r}, u^{l}\right] .\end{cases}
\end{aligned}
$$

(i) The germ $\mathcal{G}_{V V}^{o}$ is $L^{1} D$. 
(ii) The closure $\overline{\mathcal{G}_{V V}^{o}}$ of $\mathcal{G}_{V V}^{o}$ is a maximal $L^{1} D$ germ, and $\left(u^{l}, u^{r}\right) \in \overline{\mathcal{G}_{V V}^{o}} \Longleftrightarrow$

$$
\begin{aligned}
& f^{l}\left(u^{l}\right)=f^{r}\left(u^{r}\right)=: s, \text { and } \\
& \left\{\begin{array}{l}
\text { either } u^{l}=u^{r} ; \\
\text { or } u^{l}<u^{r} \text { and there exists } u^{o} \in\left[u^{l}, u^{r}\right]
\end{array}\right. \\
& \text { such that } f^{l}(z) \geqq s \text { for all } z \in\left[u^{l}, u^{o}\right] \\
& \text { and } f^{r}(z) \geqq s \text { for all } z \in\left[u^{o}, u^{r}\right] \text {; } \\
& \text { or } u^{l}>u^{r} \text { and there exists } u^{o} \in\left[u^{r}, u^{l}\right] \\
& \text { such that } f^{r}(z) \leqq s \text { for all } z \in\left[u^{r}, u^{o}\right] \\
& \text { and } f^{l}(z) \leqq s \text { for all } z \in\left[u^{o}, u^{l}\right] \text {. }
\end{aligned}
$$

(iii) We have $\mathcal{G}_{V V}^{o} \subset \mathcal{G}_{V V}^{s} \subset \overline{\mathcal{G}_{V V}^{o}}$.

(iv) Both $\mathcal{G}_{V V}^{s}$ and $\mathcal{G}_{V V}^{o}$ are definite germs with the same unique maximal $L^{1} D$ extension $\overline{\mathcal{G}_{V V}^{o}}$. The $\mathcal{G}_{V V}^{s}, \mathcal{G}_{V V}^{o}$, and $\overline{\mathcal{G}_{V V}^{o}}$ entropy solutions coincide.

Definition 5.2. In the sequel, we denote by $\mathcal{G}_{V V}$ the maximal $L^{1} D$ germ $\overline{\mathcal{G}_{V V}^{o}}$ given by (5.4). We call it the "vanishing viscosity" germ associated with the pair of functions $f^{l, r}$.

Whenever the dependence on $f^{l, r}$ should be indicated explicitly, we denote the corresponding vanishing viscosity germ by $\mathcal{G}_{V V}^{f^{l, r}}$. In [10], we will study more carefully the dependence of $\mathcal{G}_{V V}^{f^{l, r}}$ on $f^{l, r}$.

Remark 5.3. (i) Definition 5.2 allows for general continuous fluxes $f^{l, r}$. Yet it is clear that the so defined germ $\mathcal{G}_{V V}$ need not be complete. For instance, if the ranges of $f^{l, r}$ do not intersect, $\mathcal{G}_{V V}$ is empty and no $\mathcal{G}_{V V}$ entropy solution exists.

(ii) Based on different viscosity approaches, Gimse and Risebro, and then Diehl, have implicitly introduced the set of all admissible elementary solutions of (1.7). These are the elementary solutions used to construct a Riemann solver based on the "minimal jump condition" $[42,43]$ or on the " $\Gamma$-condition" [34-36]. In fact, both approaches lead to the same germ which coincides with $\mathcal{G}_{V V}$; the closure operation accounts for the difference between the minimal jump condition and the $\Gamma$-condition. For certain configurations of the fluxes $f^{l, r}$, a Riemann solver was defined for all data in [34-37,42,43]. In all these cases we conclude that $\mathcal{G}_{V V}$ is complete.

(iii) DiEHL [37] reformulated the $\Gamma$-condition from his previous works [34-36] in the following form: the pair $\left(u^{l}, u^{r}\right)$ satisfies the $\Gamma$-condition if

$$
\begin{aligned}
& f^{l}\left(u^{l}\right)=f^{r}\left(u^{r}\right) \text { and there exists } u^{o} \in \operatorname{ch}\left(u^{l}, u^{r}\right) \text { such that } \\
& \left(u^{r}-u^{o}\right)\left(f^{r}(z)-f^{r}\left(u^{r}\right)\right) \geqq 0, \quad \forall z \in \operatorname{ch}\left(u^{r}, u^{o}\right) \\
& \left(u^{o}-u^{l}\right)\left(f^{l}(z)-f^{l}\left(u^{l}\right)\right) \geqq 0, \quad \forall z \in \operatorname{ch}\left(u^{l}, u^{o}\right),
\end{aligned}
$$

where for $a, b \in \mathbb{R}, \operatorname{ch}(a, b)$ denotes the interval $[\min \{a, b\}, \max \{a, b\}]$. Clearly, (5.4) coincides with (5.5). The descriptions (5.4) and (5.5) are reminiscent of the Oleĭnik admissibility condition (for convex fluxes $f^{l, r}$ ) and of 
the "chord condition" (see $[27,41,44]$ ); as is the case with the chord condition, (5.4) and (5.5) are derived from the travelling-waves approach of [41].

Proof of Proposition 5.1. (i) The proof is entirely similar to the one of Proposition 4.11(ii). Because $f^{l, r}$ are continuous, each of the six cases in (4.13) turns out to be incompatible with the assumption that both $\left(u^{l}, u^{r}\right)$ and $\left(\hat{u}^{l}, \hat{u}^{r}\right)$ fulfill (5.3).

Notice that a less tedious proof can be obtained by passing to the limit as $\varepsilon \rightarrow 0$ in the Kato inequality which holds for equation (5.1) [indeed, recall that (1.11) follows from the Kato inequality (1.5)]. Such a proof is given in [11], for the case of Lipschitz continuous $f^{l, r}$. The general case follows by approximation.

(ii) Recall that we denote by $\mathcal{G}_{V V}$ the set of all pairs satisfying (5.4). In a first step, we show that $\mathcal{G}_{V V} \subset \overline{\mathcal{G}_{V V}^{o}}$. Since $\overline{\mathcal{G}_{V V}^{o}}$ is an $L^{1} D$ germ by (i) and Proposition 4.4(i), this implies that $\mathcal{G}_{V V}$ is an $L^{1} D$ germ.

Then in a second step, we show that $\mathcal{G}_{V V}$ contains the dual $\left(\mathcal{G}_{V V}^{o}\right)^{*}$ of $\mathcal{G}_{V V}^{o}$. According to Propositions 4.4 and 3.4, this yields the reverse inclusion $\mathcal{G}_{V V} \supset \overline{\mathcal{G}_{V V}^{o}}$ and hence the maximality of the $L^{1} D$ germ $\mathcal{G}_{V V}=\overline{\mathcal{G}_{V V}^{o}}$.

We will make repeated use of the continuity of $f^{l, r}$, without mentioning it. Step 1. Let $\left(u^{l}, u^{r}\right) \in \mathcal{G}_{V V}$. If $u^{l}=u^{r}$, then $\left(u^{l}, u^{r}\right) \in \mathcal{G}_{V V}^{o} \subset \overline{\mathcal{G}_{V V}^{o}}$. The remaining cases are symmetric; let us treat the one with $u^{l}<u^{r}$.

Take $s$ and $u^{o}$ as introduced in (5.4). Consider the function $f^{l}(\cdot)-s$ on the interval $\left[u^{o}, u^{r}\right)$. If it has a zero point, set $z^{l}:=\min \left\{z \in\left[u^{o}, u^{r}\right) \mid f^{l}(z)=s\right\}$ and $z^{r}:=\min \left\{z \in\left[z^{l}, u^{r}\right] \mid f^{r}(z)=s\right\}$ (since $f^{r}\left(u^{r}\right)=s, z^{r}$ is well defined). By construction and by (5.4), we have

- $u^{l} \leqq z^{l}, f^{l}\left(u^{l}\right)=f^{l}\left(z^{l}\right)=s$, and $f^{l} \geqq s$ on $\left[u^{l}, z^{l}\right]$;

- $z^{l} \leqq z^{r}, f^{l}\left(z^{l}\right)=f^{r}\left(z^{r}\right)=s$, and $f^{r}>s$ on the interval $\left(z^{l}, z^{r}\right)$;

- $z^{r} \leqq u^{r}, f^{r}\left(z^{r}\right)=f^{r}\left(u^{r}\right)=s$, and $f^{r} \geqq s$ on $\left[z^{r}, u^{r}\right]$.

This means that $\left(u^{l}, z^{l}\right)$ (respectively, $\left.\left(z^{r}, u^{r}\right)\right)$ is a left contact shock (respectively, a right contact shock), and $\left(z^{l}, z^{r}\right) \in \mathcal{G}_{V V}^{o}$.

Now consider the situation in which $f^{l}(\cdot)-s$ has no zero point on the interval $\left[u^{o}, u^{r}\right)$. Then $f^{l} \geqq s$ on $\left[u^{l}, u^{r}\right]$. In the case $f^{l}\left(u^{r}\right)=s,\left(u^{l}, u^{r}\right)$ is a left contact shock and $\left(u^{l}, u^{r}\right) \in \mathcal{G}_{V V}^{o}$. Otherwise, we define $z^{l}:=$ $\max \left\{z \in\left[u^{l}, u^{r}\right) \mid f^{l}(z)=s\right\}$. In this case, $\left(u^{l}, z^{l}\right)$ is a left contact shock. In addition, $\left(z^{l}, u^{r}\right) \in \mathcal{G}_{V V}^{o}$ because $z^{l}<u^{r}, f^{l}\left(z^{l}\right)=f^{r}\left(u^{r}\right)=s$, and $f^{l}>s$ on $\left(z^{l}, u^{r}\right]$.

In all the cases, by definition of the closure, we conclude that $\left(u^{l}, u^{r}\right) \in \overline{\mathcal{G}_{V V}^{o}}$. Step 2. It suffices to show that if $f^{l}\left(u^{l}\right)=f^{r}\left(u^{r}\right)$ but $\left(u^{l}, u^{r}\right) \notin \mathcal{G}_{V V}$, then there exists $\left(c^{l}, c^{r}\right) \in \mathcal{G}_{V V}^{o}$ such that

$$
q^{l}\left(u^{l}, c^{l}\right)<q^{r}\left(u^{r}, c^{r}\right) .
$$

Set $s:=f^{l, r}\left(u^{l, r}\right)$. As before, it suffices to consider the case $u^{l}<u^{r}$. Define

$$
\begin{aligned}
z^{l} & :=\sup \left\{z \in\left[u^{l}, u^{r}\right] \mid f^{l} \geqq s \text { on }\left[u^{l}, z\right]\right\}, \\
z^{r} & :=\inf \left\{z \in\left[u^{l}, u^{r}\right] \mid f^{r} \geqq s \text { on }\left[z, u^{r}\right]\right\} .
\end{aligned}
$$


If $z^{l} \geqq z^{r}$, then (5.4) holds with $u^{o}=z^{l}$, so that $\left(u^{l}, u^{r}\right)$ lies in $\mathcal{G}_{V V}$. Thus, $z^{l}<z^{r}$. Now, there are three cases to be investigated:

(a) $f^{l}$ and $f^{r}$ have a crossing point $z^{o}$ in the interval $\left(z^{l}, z^{r}\right)$ such that $f^{l, r}\left(z^{o}\right)<s$; (b) $f^{l}$ and $f^{r}$ have a crossing point $z^{o}$ in the interval $\left(z^{l}, z^{r}\right)$ such that $f^{l, r}\left(z^{o}\right) \geqq s$; (c) either $f^{l}<f^{r}$ on the interval $\left(z^{l}, z^{r}\right)$, or $f^{r}<f^{l}$ on the interval $\left(z^{l}, z^{r}\right)$.

In case (a), setting $\left(c^{l}, c^{r}\right):=\left(z^{o}, z^{o}\right)$ we obtain (5.6), because $u^{l}<c^{l}, c^{r}<u^{r}$, and $f^{l, r}\left(u^{l, r}\right)>f^{l, r}\left(z^{o}\right)$. Notice that we do have $\left(z^{o}, z^{o}\right) \in \mathcal{G}_{V V}^{o}$.

In case (b), by definition of $z^{l, r}$, there exists $\hat{s}<s$ such that $\hat{s}$ belongs to $f^{l}\left(\left[z^{l}, z^{o}\right]\right) \cap f^{r}\left(\left[z^{o}, z^{r}\right]\right)$. In this case, set $c^{l}:=\max \left\{z \in\left[z^{l}, z^{o}\right] \mid f^{l}(z)=\hat{s}\right\}$ and $c^{r}:=\min \left\{z \in\left[z^{o}, z^{r}\right] \mid f^{r}(z)=\hat{s}\right\}$. We then have (5.6) for the very same reasons as in case (a). In addition, $\left(c^{l}, c^{r}\right) \in \mathcal{G}_{V V}^{o}$, because $c^{l}<c^{r}$ and $f^{l}>\hat{s}$ on $\left(c^{l}, z^{o}\right], f^{r}>\hat{s}$ on $\left[z^{o}, c^{r}\right)$.

In case (c), the two situations are similar. Consider, for example the case $f^{l}<$ $f^{r}$ on $\left(z^{l}, z^{r}\right)$. Choose for $c^{r}$ the point of $\left[z^{l}, z^{r}\right]$ where $f^{r}$ attains its minimum value over $\left[z^{l}, z^{r}\right]$ and which is the closest one to $z^{l}$. By definition of $z^{r}, \hat{s}:=f^{r}\left(c^{r}\right)$ is smaller than $s$. Because $f^{r}\left(z^{l}\right) \geqq f^{l}\left(z^{l}\right)=s>\hat{s}$, we have $c^{r}>z^{l}$; in turn, this yields $f^{l}\left(c^{r}\right)<f^{r}\left(c^{r}\right)=\hat{s}$. Since $f^{l}\left(z^{l}\right)=s>\hat{s}$, there exists $c^{l}$ in the interval $\left(z^{l}, z^{r}\right)$ such that $f^{l}\left(c^{l}\right)=\hat{s}$. The pair $\left(c^{l}, c^{r}\right)$ fulfills (5.4). In addition, by the definition of $c^{r}$ we have $f^{r} \geqq \hat{s}$ on $\left[z^{l}, z^{r}\right] \supset\left[c^{l}, c^{r}\right]$; thus $\left(c^{l}, c^{r}\right) \in \mathcal{G}_{V V}^{o}$.

In all cases, we have constructed $\left(c^{l}, c^{r}\right) \in \mathcal{G}_{V V}^{o}$ with property (5.6).

The contradiction shows that $\left(\mathcal{G}_{V V}^{o}\right)^{*} \subset \mathcal{G}_{V V}$. Thus (ii) follows.

(iii) We first show the inclusion $\mathcal{G}_{V V}^{o} \subset \mathcal{G}_{V V}^{s}$. Let $\left(u^{l}, u^{r}\right)$ satisfy (5.3). In the case $u^{l}=u^{r}$, the standing-wave profile $W$ can be chosen constant on $\mathbb{R}$. The four other cases are symmetric. For instance, in the case $u^{l}<u^{r}$ and $f^{l}(z)>s$ for all $z \in\left(u^{l}, u^{r}\right], W$ is a continuous function that is constant (equal to $\left.u^{r}\right)$ on $[0, \infty)$. On the interval $(-\infty, 0], W$ is constructed as a solution of the autonomous ODE

$$
W^{\prime}=f^{l}(W)-f^{l}\left(u^{l}\right), \quad W(0)=u^{r} .
$$

Indeed, because $f^{l}(W)-f^{l}\left(u^{l}\right)=f^{l}(W)-s>0$ for $W$ taking values in $\left(u^{l}, u^{r}\right]$, $W$ is non-decreasing. If the graph of $W$ crosses the line $W=u^{l}$, we extend the solution by the constant value $u^{l}$ on the left from the crossing point. Otherwise, $W$ is defined on the whole interval $(-\infty, 0]$, and there exists $d:=\lim _{\xi \rightarrow-\infty} W(\xi) \in$ $\left[u^{l}, u^{r}\right]$. In this case, $f^{l}(d)-s=0$, which yields $d=u^{l}$. Therefore (5.2) holds.

Next, we show that $\mathcal{G}_{V V}^{s} \subset \overline{\mathcal{G}_{V V}^{o}}$. Let $\left(u^{l}, u^{r}\right) \in \mathcal{G}_{V V}^{s}$. The case $u^{l}=u^{r}$ is trivial; by a symmetry argument, we can assume $u^{l}<u^{r}$. The standing-wave profile $W$ in (5.2) is a continuous on $\mathbb{R}$ function which is monotone on $\mathbb{R}_{-}$and on $\mathbb{R}_{+}$. Moreover, replacing $W$ by $\max \left\{u^{l}, \min \left\{W, u^{r}\right\}\right\}$, we still have a standing-wave profile with the same properties. Then $u^{o}:=W(0)$ lies within $\left[u^{l}, u^{r}\right]$. The monotonicity of $W$ guarantees that $f^{l}-s \geqq 0$ on $\left[u^{l}, u^{o}\right]$, and $f^{r}-s \geqq 0$ on $\left[u^{o}, u^{r}\right]$. Thus $\left(u^{l}, u^{r}\right)$ fits the definition (5.4). By (ii), $\left(u^{l}, u^{r}\right) \in \overline{\mathcal{G}_{V V}^{o}}$.

(iv) The claim follows readily from (ii), (iii), and Proposition 4.4 (iii), (iv).

Now we compare $\mathcal{G}_{V V}$ with the known germs studied in Section 4 and in [34$37,42,43]$. Let us emphasize that in many cases studied in these papers, $\mathcal{G}_{V V}$ turns 
out to be complete. The completeness of $\mathcal{G}_{V V}$ will be further studied in Theorem 6.1 (see Section 6).

Remark 5.4. (i) With the help of the characterizations (4.11), (5.3), (5.4) of $\mathcal{G}_{K R T}, \mathcal{G}_{V V}^{o}$, and $G_{V V}$, respectively, we easily see that $\mathcal{G}_{V V}^{o} \subset \mathcal{G}_{K R T}=\mathcal{G}_{V V}$ whenever the crossing condition (4.8) holds. In this case, the $\mathcal{G}_{V V}$ and $\mathcal{G}_{K R T}$ entropy solutions coincide.

(ii) Consider the case of bell-shaped fluxes (4.14) treated in Section 4.8.

- First assume $u_{o}^{r} \leqq u_{o}^{l}$ and that there exists a crossing point $u_{\chi} \in\left[u_{o}^{r}, u_{o}^{l}\right]$ between $f^{l}$ and $f^{r}$ (in this case, the crossing condition (4.8) holds). Then $\mathcal{G}_{V V}$ contains the definite germ $\mathcal{G}_{\left(u_{\chi}, u_{\chi}\right)}$. Thus the $\mathcal{G}_{\left(u_{\chi}, u_{\chi}\right)}, \mathcal{G}_{V V}$, and $\mathcal{G}_{K R T}$ entropy solutions coincide. In addition, since $\left(\mathcal{G}_{\left(u_{\chi}, u_{\chi}\right)}\right)^{*}$ is complete, $\mathcal{G}_{V V}$ is complete.

- If, on the other hand, a crossing point $u_{\chi}$ exists but $u_{o}^{l} \leqq u_{\chi} \leqq u_{o}^{r}$ (this is the "bad crossing" case), then the $\mathcal{G}_{V V}$-entropy solutions coincide with the entropy solutions for the germ $\mathcal{G}_{(A, B)}$ with $(A, B)$ corresponding to $s_{(A, B)}=s_{\max }$, where

$$
s_{\max }:=\max \left\{s \in \mathbb{R}_{+} \mid \exists(A, B) \in\left[u_{o}^{l}, 1\right] \times\left[0, u_{o}^{r}\right] \text { s.t. } f^{l}(A)=f^{r}(B)=s\right\} .
$$

Also in the case where the fluxes do not cross within $(0,1), \mathcal{G}_{V V}=\mathcal{G}_{(A, B)}$ with $s_{(A, B)}=s_{\max }$. In both cases, $\mathcal{G}_{V V}$ is complete because $\left(\mathcal{G}_{(A, B)}\right)^{*}$ is complete. Let us stress that in the non-crossing case, $\mathcal{G}_{K R T}$ coincides with $\mathcal{G}_{V V}$, whereas in the case of a "bad crossing", $\mathcal{G}_{K R T} \backslash \mathcal{G}_{V V} \neq \emptyset$.

In conclusion, following DiEHL [34-36] and BürgER ET AL. [22], let us mention that the germ $\overline{\mathcal{G}_{V V}^{o}}$ also arises from the more general regularization-viscosity approach

$$
u_{t}+\mathfrak{f}^{\delta}(x, u)_{x}=\varepsilon u_{x x} \text { in } \mathcal{D}^{\prime}\left(\mathbb{R}_{+} \times \mathbb{R}\right),
$$

where $\mathfrak{f}^{\delta}$ is a continuous (in $x$ ) approximation to $\mathfrak{f}$.

Proposition 5.5. Suppose $f^{l, r}$ are locally Lipschitz continuous. Let $\left(f^{\delta}(\cdot, \cdot)\right)_{\delta>0}$ be a family of continuous functions approximating $\mathfrak{f}$ in (1.7) in the following sense:

- $f^{\delta}(x, z)$ is of the form $F(x / \delta, z)$ with some fixed function $F$;

- for all $y \in \mathbb{R}$, there exists $\alpha_{y} \in[0,1]$ such that $F(y, \cdot)=\alpha_{y} f^{l}(\cdot)+(1-$ $\left.\alpha_{y}\right) f^{r}(\cdot)$;

- for $y \leqq-1, \alpha_{y}=1$ (that is, $\left.F(y, \cdot) \equiv f^{l}(\cdot)\right)$; similarly, for $y \geqq 1, F(y, \cdot) \equiv$ $f^{r}(\cdot)$.

Let $\left(u^{l}, u^{r}\right) \in \mathcal{G}_{V V}^{o}$. Denote by L the Lipschitz constant of $f^{l, r}$ on the interval between $u^{l}$ and $u^{r}$.

Then, whenever $\delta / \varepsilon \leqq 2 / L$, there exists a stationary solution $u(t, x)=u^{\varepsilon, \delta}(x)$ of (5.8) satisfying $\lim _{x \rightarrow-\infty} u^{\varepsilon, \delta}(x)=u^{l}, \lim _{x \rightarrow \infty} u^{\varepsilon, \delta}(x)=u^{r}$. In addition, as $\varepsilon \downarrow 0$, with $0<\delta \leqq \frac{2}{L} \varepsilon$, $u^{\varepsilon, \delta}$ converges almost everywhere on $\mathbb{R}$ and in $L_{\mathrm{loc}}^{1}(\mathbb{R})$ to the elementary stationary solution $u^{l} 1_{\{x<0\}}+u^{r} 1_{\{x>0\}}$ of (1.7). 
Proof. Let $\left(u^{l}, u^{r}\right) \in \mathcal{G}_{V V}^{o}$. The case $u^{l}=u^{r}$ is trivial since constants solve (5.8). The other cases are symmetric, so let us consider only the one with $u^{l}<u^{r}$ and $f^{l}>s$ on the interval $\left(u^{l}, u^{r}\right]$.

Setting $y=x / \delta, u^{\varepsilon, \delta}(x)=U(x / \delta)$, we are reduced to the problem

$$
\varepsilon U^{\prime}(y)=\delta(F(y, U(y))-s), \quad U(-\infty)=u^{l}, U(\infty)=u^{r} .
$$

In view of the assumptions on $F$ and also the ratio $\delta / \varepsilon$, the (constant) function $m_{+}: y \in(-\infty, 1] \mapsto u^{r}$ is a subsolution of the ODE $U^{\prime}=\delta / \varepsilon(F(y, U)-s)$; and the function $m_{-}: y \in(-\infty, 1] \mapsto \min \left\{u^{l}, u^{r}+L \delta / \varepsilon(y-1)\right\}$ is a supersolution of the same ODE. By the classical ODE existence and comparison result, there exists a solution of the ODE on $(-\infty, 1]$ with $U(1)=u^{r}$, and we have $m_{-} \leqq U \leqq m_{+}$. In particular, $U(\cdot)$ takes values in $\left(u^{l}, u^{r}\right]$ and satisfies the autonomous equation $U^{\prime}=\delta / \varepsilon\left(f^{l}(U)-s\right)$ on $(-\infty,-1)$. Since by assumption, $f^{l}>s$ on $\left(u^{l}, u^{r}\right]$, $U(\cdot)$ admits a limit as $y \rightarrow-\infty$ which necessarily equals $u^{l}$. Finally, extending $U$ by the constant value $u^{r}$ on $[1, \infty)$, we obtain a solution to problem (5.9).

For the proof of convergence of the solutions $u^{\varepsilon, \delta}(x)=U(x / \delta)$, we replace $m_{+}(\cdot)$ on $(-\infty,-1]$ by the solution $V(\cdot)$ of $V^{\prime}=\delta / \varepsilon\left(f^{l}(V)-s\right)$ with the initial datum $V(-1)=u^{r}$; with the same arguments as above, such a solution exists, it tends to $u^{l}$ as $y \rightarrow-\infty$, and it bounds $U(\cdot)$ from above. By assumptions, we have $\delta \leqq 2 \varepsilon / L \rightarrow 0$ as $\varepsilon \rightarrow 0$. For all $x<0$, for all sufficiently small $\varepsilon$, the quantity $\left|u^{\bar{\varepsilon}, \delta}(x)-u^{l}\right|$ is upper bounded by $\left|V(x / \delta)-u^{l}\right|$ which converges to zero. For $x>0$, we simply have $u^{\varepsilon, \delta}(x)=u^{r}$ whenever $\delta \leqq x$. We conclude the pointwise convergence of $u^{\varepsilon, \delta}$ to the profile (1.10); since all these functions take values within $\left[u^{l}, u^{r}\right]$, the $L_{\text {loc }}^{1}$ convergence follows.

\section{Some existence and convergence results}

In this section, for a fixed germ $\mathcal{G}$, we study the existence of $\mathcal{G}$-entropy solutions and convergence of various vanishing viscosity and numerical approximations.

The first result concerns the vanishing viscosity germ $\mathcal{G}_{V V}$, which was described in Section 5. The main assumption for the existence result is the availability of a uniform $L^{\infty}\left(\mathbb{R}_{+} \times \mathbb{R}\right)$ estimate for solutions $u^{\varepsilon}$ of (5.1) with a given initial datum $u_{0} \in L^{\infty}(\mathbb{R})$. We also impose some additional nonlinearity and Lipschitz continuity assumptions on the fluxes $f^{l, r}$. For the sake of simplicity, we restrict our attention to fluxes satisfying $f^{l, r}(0)=0=f^{l, r}(1)$ [the bell-shaped fluxes (4.14) offer one example] and an initial condition $u_{0}$ with values in $[0,1]$; thus solutions are automatically bounded, because they take values in $[0,1]$.

Recall that in the setting (4.14) of bell-shaped fluxes with $u_{o}^{l} \leqq u_{\chi} \leqq u_{o}^{r}$, the germ $\mathcal{G}_{V V}$ coincides with $\left(\mathcal{G}_{\left(u_{\chi}, u_{\chi}\right)}\right)^{*}$; the other maximal $L^{1} D$ germs $\left(\mathcal{G}_{(A, B)}\right)^{*}$ are corresponding to other choices of the connection $(A, B)$. For an arbitrary but fixed connection $(A, B)$, using a specially fabricated artificial viscosity we prove convergence of viscosity approximations to the $\mathcal{G}_{(A, B)}$-entropy solution.

Then we look at a general complete $L^{1} D$ germ $\mathcal{G}$ for a pair of locally Lipschitz continuous functions $f^{l, r}$. We construct solutions by showing convergence of a suitably adapted monotone three-point finite volume scheme. For 
these results, the uniform $B V_{\text {loc }}$ estimates away from the interface $\{x=0\}$ are of importance (see [20,22]). As previously, we need a uniform $L^{\infty}$ bound on the approximations, which actually comes for free from the completeness of $\mathcal{G}$ (see Proposition 3.20).

Finally, we prove convergence of uniformly $L^{\infty}$ bounded (thus weakly compact) sequences of approximate $\mathcal{G}$-entropy solutions, without utilizing $B V$ type a priori estimates and assumptions that ensure strong compactness. For this purpose, we have to assume that $\mathcal{G}$ is a maximal $L^{1} D$ germ for which existence is already known (see Section 1.4). Then, assuming that the approximation procedure is compatible with the germ $\mathcal{G}$ (that is, that the elementary stationary solutions selected by the germ $\mathcal{G}$ are obtained as limits of the approximation) and that an appropriate Kato inequality holds for the approximate solutions, we deduce the convergence of the approximating procedure.

\subsection{The standard vanishing viscosity approach}

To be specific, let us work with the vanishing viscosity germ $\mathcal{G}_{V V}$ in Definition 5.2, and note that according to Proposition 5.1(iv), we can replace $\mathcal{G}_{V V}$ by $\mathcal{G}_{V V}^{s}$ or by $\mathcal{G}_{V V}^{o}$.

Theorem 6.1. Suppose $f^{l, r}:[0,1] \longrightarrow \mathbb{R}$ are Lipschitz continuous functions that are not affine on any interval $I \subset[0,1]$. Moreover, assume $f^{l, r}(0)=0=f^{l, r}(1)$. Then for all $u_{0}$ which is measurable and takes values in $[0,1]$, there exists a unique $\mathcal{G}_{V V}$-entropy solution to problem (1.7),(1.2). In particular, the germ $\mathcal{G}_{V V}$ is definite and complete. In addition, the $\mathcal{G}_{V V}$-entropy solutions are the vanishing viscosity limits in the following sense. For each $\varepsilon>0$, there exists a weak solution $u^{\varepsilon} \in L_{\mathrm{loc}}^{2}\left(\mathbb{R}_{+} ; H_{\mathrm{loc}}^{1}(\mathbb{R})\right)$ of the viscous problem $(5.1)$ with any measurable initial data $\left.u^{\varepsilon}\right|_{t=0}=u_{0}^{\varepsilon}$ taking values in $[0,1]$. If we moreover assume $u_{0}^{\varepsilon} \rightarrow u_{0}$ almost everywhere on $\mathbb{R}$, then $u^{\varepsilon}$ converge almost everywhere on $\mathbb{R}_{+} \times \mathbb{R}$ to the unique $\mathcal{G}_{V V}$-entropy solution of (1.7), (1.2).

A multi-dimensional analogue of Theorem 6.1 holds, see [10,11].

Remark 6.2. (i) The assumptions $f^{l, r}(0)=0=f^{l, r}(1)$ and $u_{0}^{\varepsilon}(x) \in[0,1]$ are put forward to ensure that the family $\left(u^{\varepsilon}\right)_{\varepsilon>0}$ is bounded in $L^{\infty}$. Indeed, Theorem 6.1 remains true if the $L^{\infty}$ bound is provided by some other means. This boundedness assumption is important. For example, in situations where the graphs of $f^{l}$ and $f^{r}$ do not intersect, it is then clear that (1.7) has no solution and consequently $\left\|u^{\varepsilon}\right\|_{\infty} \rightarrow \infty$ as $\varepsilon \rightarrow 0$.

(ii) On the contrary, the Lipschitz continuity assumption on $f^{l, r}$ is needed only to establish the existence of a solution semigroup for viscous problem (5.1) that satisfies the Kato inequality. This assumption can be bypassed, thanks to a regularization of $f^{l, r}$. We then establish the existence of a (possibly nonunique) solution $u^{\varepsilon}$ to (5.1), (1.2) with merely continuous functions $f^{l, r}$, and the convergence of the sequence $\left(u^{\varepsilon}\right)_{\varepsilon>0}$ to the unique $\mathcal{G}_{V V}$-entropy solution of problem (1.7), (1.2). 
(iii) As pointed out in Corollary 6.6, the assumption of non-degeneracy of $f^{l, r}$ imposed in Theorem 6.1 can be dropped; but the proof becomes much more indirect, involving a beforehand justification of the existence of $\mathcal{G}_{V V}$-entropy solutions (for this, we use a numerical scheme, see Theorem 6.4). Moreover, in Corollary 6.6 we do not prove that the germ $\mathcal{G}_{V V}$ is complete, but we take completeness as a hypothesis.

Proof of Theorem 6.1. Extend $f^{l, r}$ by zero outside [0, 1]. Let $T$ denote a generic positive number. The following a priori estimate is easily obtained:

$$
\begin{aligned}
\int_{\mathbb{R}} & \frac{1}{2}\left(u^{\varepsilon}(T, \cdot)\right)^{2}+\varepsilon \int_{0}^{T} \int_{\mathbb{R}}\left|u_{x}^{\varepsilon}\right|^{2} \\
\leqq & \int_{\mathbb{R}} \frac{1}{2}\left(u^{\varepsilon}(0, \cdot)\right)^{2} \\
& +\int_{0}^{T} \int_{-\infty}^{0}\left(\int_{0}^{u^{\varepsilon}} f^{l}(z) d z\right)_{x}+\int_{0}^{T} \int_{0}^{\infty}\left(\int_{0}^{u^{\varepsilon}} f^{r}(z) d z\right)_{x} \\
\leqq & C\left(T,\left\|u_{0}^{\varepsilon}\right\|_{2}, f^{l, r}\right),
\end{aligned}
$$

where $u^{\varepsilon} \in C\left(\mathbb{R}_{+} ; H^{1}(\mathbb{R})\right)$ is a solution of (5.1). Thanks to this estimate, it can be shown by the classical Galerkin technique (see, for example Lions [59]) that for any $\varepsilon>0$ and $u_{0}^{\varepsilon} \in L^{2}(\mathbb{R})$ there exists a solution $u^{\varepsilon} \in C\left(\mathbb{R}_{+} ; H^{1}(\mathbb{R})\right)$ to the Cauchy problem for the parabolic equation (5.1). What's more, the Kato inequality holds for (5.1). Indeed, assuming $\left(u_{0}^{\varepsilon}-\hat{u}_{0}^{\varepsilon}\right)^{+}$belongs to $\in L^{1}(\mathbb{R})$, let $u^{\varepsilon}, \hat{u}^{\varepsilon} \in L_{\text {loc }}^{2}\left(\mathbb{R}_{+} ; H_{\text {loc }}^{1}(\mathbb{R})\right)$ be two weak solutions of (5.1) corresponding to the initial data $u_{0}^{\varepsilon}, \hat{u}_{0}^{\varepsilon} \in L^{\infty}(\mathbb{R})$, respectively. For $\alpha<0$, let us introduce a Lipschitz continuous approximation $H_{\alpha}$ of the $\operatorname{sign}^{+}(\cdot)$ function:

$$
H_{\alpha}(z):=\min \left\{\frac{z^{+}}{\alpha}, 1\right\}, \quad z \in \mathbb{R},
$$

and use the test function $H_{\alpha}\left(u^{\varepsilon}-\hat{u}^{\varepsilon}\right) \xi, \xi \in \mathcal{D}([0, \infty) \times \mathbb{R})$, in the weak formulation of (5.1). Notice that, due to the Lipschitz assumption on $f^{l}$,

$$
\begin{aligned}
& \left|\int_{\mathbb{R}_{+}} \int_{\mathbb{R}_{-}}\left(f^{l}\left(u^{\varepsilon}\right)-f^{l}\left(\hat{u}^{\varepsilon}\right)\right)\left(H_{\alpha}\right)^{\prime}\left(u^{\varepsilon}-\hat{u}^{\varepsilon}\right)\left(u^{\varepsilon}-\hat{u}^{\varepsilon}\right)_{x} \xi\right| \\
& \quad \leqq\left\|\left(f^{l}\right)^{\prime}\right\|_{L^{\infty}\|\xi\|_{L^{\infty}} \iint_{\left\{0<u^{\varepsilon}-\hat{u}^{\varepsilon}<\alpha\right\}}\left|\left(u^{\varepsilon}-\hat{u}^{\varepsilon}\right)_{x}\right| \longrightarrow 0 \quad \text { as } \alpha \rightarrow 0,}
\end{aligned}
$$

thanks to (6.1); and the same holds with $\mathbb{R}_{-}, f^{l}$ replaced with $\mathbb{R}_{+}, f^{r}$. Hence as $\alpha \rightarrow 0$, we deduce the Kato inequality: for all $\xi \in \mathcal{D}([0, \infty) \times \mathbb{R}), \xi \geqq 0$,

$$
\begin{aligned}
& \int_{\mathbb{R}_{+}} \int_{\mathbb{R}}\left(\left(u^{\varepsilon}-\hat{u}^{\varepsilon}\right)^{+} \xi_{t}+\mathfrak{q}_{+}\left(x, u^{\varepsilon}, \hat{u}^{\varepsilon}\right) \xi_{x}\right) \\
& \quad+\int_{\mathbb{R}}\left(u_{0}^{\varepsilon}-\hat{u}_{0}^{\varepsilon}\right)^{+} \xi(0, \cdot) \geqq \varepsilon \int_{\mathbb{R}_{+}} \int_{\mathbb{R}}\left[\left(u^{\varepsilon}-\hat{u}^{\varepsilon}\right)^{+}\right]_{x} \xi_{x} ;
\end{aligned}
$$


where we have written $\mathfrak{q}_{+}$for the semi-Kruzhkov entropy flux:

$\mathfrak{q}_{+}(x, z, k):=\operatorname{sign}^{+}(z-k)\left(\left(f^{l}(z)-f^{l}(k)\right) \mathbb{1}_{\{x<0\}}+\left(f^{r}(z)-f^{r}(k)\right) \mathbb{1}_{\{x>0\}}\right)$.

In this inequality, using, for example the technique of MALIKI and TouRÉ [62] we can let $\xi$ converge to the characteristic function of $[0, T) \times \mathbb{R}$ in such a way that the terms with $\xi_{x}$ vanish as $\varepsilon \rightarrow 0$. Then we get the $L^{1}$ contraction and comparison inequality

$$
\text { for almost every } t>0, \quad \int_{\mathbb{R}}\left(u^{\varepsilon}-\hat{u}^{\varepsilon}\right)^{+}(t) \leqq \int_{\mathbb{R}}\left(u_{0}^{\varepsilon}-\hat{u}_{0}^{\varepsilon}\right)^{+} .
$$

Inequality (6.4) ensures the uniqueness of a weak solution in $L_{\text {loc }}^{2}\left(\mathbb{R}_{+} ; H_{\text {loc }}^{1}(\mathbb{R})\right)$ of the Cauchy problem for the parabolic equation (5.1). It also yields the comparison principle. Keeping in mind that, under our assumptions, the constants 0 and 1 are evident solutions of (5.1), we derive the maximum principle: for data $u_{0}^{\varepsilon}$ taking values in $[0,1], 0 \leqq u^{\varepsilon} \leqq 1$ holds almost everywhere on $\mathbb{R}_{+} \times \mathbb{R}$.

Now we are in a position to extend the existence result for (5.1) to a general initial function $u_{0}$ taking values in $[0,1]$. Indeed, following AMMAR and WITTBOLD [6] we can take the bi-monotone approximation of $u_{0} \in L^{\infty}(\mathbb{R})$ by bounded compactly supported functions, namely,

$$
\left(u_{0}\right)_{m, n}:=\min \left\{\left(u_{0}\right)^{+}, n\right\} \mathbb{1}_{\{|x|<n\}}-\min \left\{\left(u_{0}\right)^{-}, m\right\} \mathbb{1}_{\{|x|<m\}} .
$$

We deduce the existence of a weak solution to (5.1). Moreover, thanks to the monotone convergence theorem, the obtained solutions still fulfill (6.3) and (6.4). Finally, the solutions belong to $L_{\text {loc }}^{2}\left(\mathbb{R}_{+} ; H_{\text {loc }}^{1}(\mathbb{R})\right)$, thus the uniqueness result applies.

Now, let us justify the convergence of $u^{\varepsilon}$ to a $\mathcal{G}_{V V}$-entropy solution. Take a sequence of data $u_{0}^{\varepsilon}$ as in the statement of the theorem. By the non-degeneracy assumption on $f^{l, r}$ and strong precompactness results of [70,73], applied separately in the domains $\{x>0\}$ and $\{x<0\}$, we deduce that there exists a (not labelled) sequence $\varepsilon \downarrow 0$ such that $u^{\varepsilon}$ converge to some limit $u$ in $L_{\text {loc }}^{1}\left(\mathbb{R}_{+} \times \mathbb{R}\right)$. By the dominated convergence theorem, we also have the $L_{\text {loc }}^{1}$ convergence of $u_{0}^{\varepsilon}$ to $u_{0}$. Passing to the limit in the distributional formulation of the Cauchy problem for (5.1), we find that $u$ is a weak solution of (1.7), (1.2). Following [54], we also deduce the Kruzhkov entropy inequalities in the domains $\{x>0\}$ and $\{x<0\}$. In particular, the existence of strong traces $\gamma^{l, r} u$ on $\{x=0\}$ follows.

Finally, notice that for any pair $u, \hat{u}$ obtained as the vanishing viscosity limit with the same extracted subsequence $\varepsilon \rightarrow 0$, we can pass to the limit in the Kato inequality (6.3), where the right-hand side vanishes due to a uniform bound on $\varepsilon\left|u_{x}^{\varepsilon}\right|^{2}$ in $L_{\text {loc }}^{1}\left(\mathbb{R}_{+} \times \mathbb{R}\right)$. To conclude the proof, it remains to notice that, by the Definition (5.2) of $\mathcal{G}_{V V}^{s}$, the elementary solutions $\hat{u}=c^{l} 1_{\{x<0\}}+c^{r} 1_{\{x>0\}},\left(c^{l}, c^{r}\right) \in \mathcal{G}_{V V}^{s}$, are obtained as vanishing viscosity limits. Passing to the limit in the corresponding Kato inequalities written for $\varepsilon>0$, we infer the entropy inequalities (3.8) with $\left(c^{l}, c^{r}\right) \in \mathcal{G}_{V V}^{s}$ and zero remainder term $\mathcal{R}_{\mathcal{G}}$. According to Proposition 3.17 and Definition 3.15, $u$ is a $\mathcal{G}_{V V}^{S}$-entropy solution of (1.7), (1.2). By Proposition 5.1(iv), $\left(\mathcal{G}_{V V}^{s}\right)^{*}$ coincides with the maximal $L^{1} D$ germ $\mathcal{G}_{V V}$, thus we conclude that $u$ is the 
unique $\mathcal{G}_{V V}$-entropy solution of (1.7), (1.2). The uniqueness of the accumulation point ensures that all sequences converge to the same limit $u$.

It remains to notice that we have obtained a solution for every measurable initial function $u_{0}$ with values in $U=[0,1]$. In particular, for all Riemann initial data $u_{0}$ in (1.8), there exists a $\mathcal{G}_{V V}$-entropy solution. According to Remark 3.13, the germ $\mathcal{G}_{V V}$ is complete.

\subsection{The vanishing viscosity approach adapted to $(A, B)$-connections}

In this section we work with bell-shaped fluxes as defined in (4.14). Recall that any complete maximal $L^{1} D$ germ is of the form $\left(\mathcal{G}_{(A, B)}\right)^{*}$, where $\mathcal{G}_{(A, B)}:=$ $\{(A, B)\}$ is a definite germ and the connection $(A, B)$ is a pair satisfying

$$
(A, B) \in\left[u_{o}^{l}, 1\right] \times\left[0, u_{o}^{r}\right] \text { and } f^{l}(A)=f^{r}(B)=: s_{(A, B)} .
$$

The explicit description of $\left(G_{(A, B)}\right)^{*}$ is given by formula (4.15).

Our goal is to construct $\mathcal{G}_{(A, B)}$-entropy solutions by the vanishing viscosity method; clearly, the choice of viscosity must be adapted to the connection $(A, B)$. For the Buckley-Leverett equation with a flux that is discontinuous at $x=0$, KAASSCHIETTER [48] gives a physically motivated viscosity of the form

$$
\varepsilon\left(\lambda(x, u) p_{c}(u)_{x}\right)_{x},
$$

where $p_{c}(\cdot)$ is the capillary pressure and $\lambda(x, \cdot)=\lambda^{l}(\cdot) 1_{\{x<0\}}+\lambda^{r}(\cdot) 1_{\{x>0\}}$ is the mobility function, discontinuous at $\{x=0\}$. This choice corresponds to the particular connection $(A, B)$ such that $s_{(A, B)}=s_{\max }$, see (5.7).

Our approach is more academic. We fix an arbitrary connection $(A, B)$ and construct an artificial "adapted viscosity" of the form

$$
\varepsilon(a(x, u))_{x x}
$$

such that the stationary solution $c(x):=A 1_{\{x<0\}}+B 1_{\{x>0\}}$ of the limit equation (1.7) is also a solution of the viscous problem

$$
\begin{aligned}
& u_{t}+\mathfrak{f}(x, u)_{x}=\varepsilon a(x, u)_{x x}, \\
& \mathfrak{f}(x, z)=\left\{\begin{array}{ll}
f^{l}(z), & x<0, \\
f^{r}(z), & x>0 .
\end{array} \quad a(x, z)= \begin{cases}a^{l}(z), & x<0, \\
a^{r}(z), & x>0 .\end{cases} \right.
\end{aligned}
$$

In the sequel, by a weak solution of (6.6) we mean a function $u \in L^{\infty}\left(\mathbb{R}_{+} \times\right.$ $\mathbb{R})$ satisfying $(6.6)$ in the sense of distributions and such that $w(\cdot):=a(\cdot, u(\cdot))$ belongs to $L_{\text {loc }}^{2}\left(\mathbb{R}_{+} ; H_{\text {loc }}^{1}(\mathbb{R})\right)$. For (6.6) to be parabolic, the functions $a^{l, r}$ should be strictly increasing on $[0,1]$; and it is convenient to ask that $\inf _{z \in[0,1]}\left(a^{l, r}\right)^{\prime}(z)>0$. Because $f^{l, r}(0)=0=f^{l, r}(1)$ by assumption, it is convenient to require the constants $u \equiv 0$ and $u \equiv 1$ to be solutions of (6.6) for all $\varepsilon>0$.

For example, any continuous functions $a^{l, r}$ such that

$$
a^{l, r} \text { are strictly monotone on }[0,1], \text { and }\left\{\begin{array}{l}
a^{l}(0)=0=a^{r}(0), \\
a^{l}(A)=\kappa=a^{r}(B), \\
a^{l}(1)=1=a^{r}(1),
\end{array}\right.
$$


with $\kappa \in(0,1)$, do satisfy the above requirements, except with $(A, B)=(1,0)$, in which case we take $\kappa=0$. For example, we can interpolate the values (6.7) to produce piecewise affine and strictly increasing examples of such functions $a^{l, r}$ defined on $[0,1]$. Notice that, by choosing $\kappa$ appropriately, for the connection $\left(u_{\chi}, u_{\chi}\right)$ we obtain the standard viscosity $\varepsilon u_{x x}$ studied in the previous section; under assumption (4.14), we actually have $\left(\mathcal{G}_{\left(u_{\chi}, u_{\chi}\right)}\right)^{*}=\mathcal{G}_{V V}$.

Now we will prove that the $\mathcal{G}_{(A, B)}$-entropy solutions of (1.7) can be obtained as the limits of the vanishing adapted viscosity approximations defined by (6.6). More precisely, we have the following analogue of Theorem 6.1, which represents a reinterpretation and improvement of the results of BÜRGER ET AL. [22].

Theorem 6.3. Assume that $f^{l, r}$ are Lipschitz continuous functions of the form (4.14). In addition, assume that $f^{l}$ and $f^{r}$ are not affine on any interval $I \subset[0,1]$. Let $(A, B)$ be a given connection of the form (6.5). Then, for each measurable initial function $u_{0}$ taking values in $[0,1]$, there exists a unique $\mathcal{G}_{(A, B)}$-entropy solution of (1.7), (1.2). Moreover, consider $a^{l, r}$ satisfying (6.7) and set

$$
a(x, \cdot)=a^{l}(\cdot) 1_{\{x<0\}}+a^{r}(\cdot) 1_{\{x>0\}} ;
$$

assume in addition that $\left(a^{l, r}\right)^{\prime} \geqq$ const $>0$ almost everywhere on [0,1]. Let $\left(u_{0}^{\varepsilon}\right)_{\varepsilon>0}$ be a family of measurable functions taking values in $[0,1]$ such that $u_{0}^{\varepsilon} \rightarrow u_{0}$ almost everywhere on $\mathbb{R}$. For each $\varepsilon>0$, there exists a weak solution $u^{\varepsilon}$ of the adapted viscosity regularized problem (6.6) with initial data $\left.u^{\varepsilon}\right|_{t=0}=u_{0}^{\varepsilon}$. The sequence $\left(u^{\varepsilon}\right)_{\varepsilon>0}$ converges almost everywhere on $\mathbb{R}_{+} \times \mathbb{R}$ to the unique $\mathcal{G}_{(A, B)}$-entropy solution of (1.7), (1.2).

Proof. We will focus on the existence of solutions to the adapted viscous problems and the convergence of these solutions as the viscosity parameter tends to zero. The proof is very similar to the proof of Theorem 6.1. We will argue in terms of the unknown $w(t, x):=a(x, u(t, x))$. Then

$$
u(t, x)=b(x, w(t, x)), \quad b(x, \cdot)=\left(a^{l}\right)^{-1}(\cdot) 1_{\{x<0\}}+\left(a^{r}\right)^{-1}(\cdot) 1_{\{x>0\}},
$$

and the Cauchy problem for (6.6) is equivalent to

$$
b(x, w)_{t}+\mathfrak{f}(x, b(x, w))_{x}=\varepsilon w_{x x}, \quad w(0, x)=w_{0}^{\varepsilon}:=a\left(x, u_{0}^{\varepsilon}(x)\right) .
$$

Existence for (6.8) is obtained by proving convergence of Galerkin approximations along the lines of ALT and LucKHAus [5]. Indeed, set $\mathcal{B}(x, r):=\int_{0}^{r} b(x, z) d z$. In the same way as for $(6.1)$, assuming that $\mathcal{B} \circ w_{0}^{\varepsilon}(\cdot):=\mathcal{B}\left(\cdot, w_{0}^{\varepsilon}(\cdot)\right)$ belongs to $L^{1}(\mathbb{R})$ and using the weak chain rule [5], we obtain the a priori estimate

$$
\begin{array}{rl}
\int_{\mathbb{R}} & \mathcal{B}\left(\cdot, w^{\varepsilon}(T, \cdot)\right)+\varepsilon \int_{0}^{T} \int_{\mathbb{R}}\left|w_{x}^{\varepsilon}\right|^{2} \\
\leqq & \int_{\mathbb{R}} \mathcal{B}\left(\cdot, u^{\varepsilon}(0, \cdot)\right) \\
& \quad+\int_{0}^{T} \int_{-\infty}^{0}\left(\int_{0}^{u^{\varepsilon}} f^{l}(z) a^{l}(z) d z\right)_{x}+\int_{0}^{T} \int_{0}^{\infty}\left(\int_{0}^{u^{\varepsilon}} f^{r}(z) a^{r}(z) d z\right)_{x} \\
\leqq & C\left(T,\left\|\mathcal{B} \circ w_{0}^{\varepsilon}\right\|_{L^{1}(\mathbb{R})}, f^{l, r}, a^{l, r}\right) .
\end{array}
$$


This estimate and the compactness technique of [5] ensure the convergence of the Galerkin approximations to a weak solution $w^{\varepsilon}$ of (6.8) with the properties

$$
\begin{aligned}
& \mathcal{B} \circ w^{\varepsilon} \in L^{\infty}\left(\mathbb{R}_{+} ; L^{1}(\mathbb{R})\right), \quad w^{\varepsilon} \in L_{\mathrm{loc}}^{2}\left(\mathbb{R}_{+} ; H^{1}(\mathbb{R})\right), \\
& \left(b \circ w^{\varepsilon}\right)_{t} \in L_{\mathrm{loc}}^{2}\left(\mathbb{R}_{+} ; H^{-1}(\mathbb{R})\right), \quad\left(b \circ w^{\varepsilon}\right)(0, \cdot)=b \circ w_{0}^{\varepsilon} .
\end{aligned}
$$

As in the proof of Theorem 6.1, the assumption $\mathcal{B} \circ w_{0}^{\varepsilon} \in L^{1}(\mathbb{R})$ can be dropped, thanks to the bi-monotone approximation of $w_{0}^{\varepsilon}$ by compactly supported bounded functions

$$
\left(w_{0}^{\varepsilon}\right)_{m, n}:=\min \left\{\left(w_{0}^{\varepsilon}\right)^{+}, n\right\} \mathbb{1}_{\{|x|<n\}}-\min \left\{\left(w_{0}^{\varepsilon}\right)^{-}, m\right\} \mathbb{1}_{\{|x|<m\}}
$$

(see [6]) and thanks to the comparison principle that we now justify. Indeed, let $\hat{w}^{\varepsilon}$ be a solution of the same equation corresponding to the initial datum $\hat{w}_{0}^{\varepsilon}$. Take $\xi \in \mathcal{D}([0, \infty) \times \mathbb{R}), \xi \geqq 0$, and $H_{\alpha}$ given by (6.2). Utilizing the test function $H_{\alpha}\left(w^{\varepsilon}-\hat{w}^{\varepsilon}\right) \xi$, using the doubling-of-variables technique in time only, as in Отто [68], and taking into account that $f^{l, r}$ and $b^{l, r}$ are Lipschitz continuous (see the proof of Theorem 6.1), we deduce the Kato inequality

$$
\begin{aligned}
& \int_{\mathbb{R}_{+}} \int_{\mathbb{R}}\left(\left(b \circ w^{\varepsilon}-b \circ \hat{w}^{\varepsilon}\right)^{+} \xi_{t}+\mathfrak{q}_{+}\left(x ; b \circ w^{\varepsilon}, b \circ \hat{w}^{\varepsilon}\right) \xi_{x}\right) \\
& \quad+\int_{\mathbb{R}}\left(b \circ w_{0}^{\varepsilon}-b \circ \hat{u}_{0}^{\varepsilon}\right)^{+} \xi(0, \cdot) \geqq \varepsilon \int_{\mathbb{R}_{+}} \int_{\mathbb{R}}\left(w_{x}^{\varepsilon}-\hat{w}_{x}^{\varepsilon}\right) \xi_{x}
\end{aligned}
$$

here $\mathfrak{q}_{+}$has the same meaning as in (6.3). With the technique of [62], we can let $\xi$ converge to the characteristic function of $[0, T) \times \mathbb{R}$ and derive the contraction and comparison inequality

$$
\text { for almost every } t>0, \quad \int_{\mathbb{R}}\left(u^{\varepsilon}-\hat{u}^{\varepsilon}\right)^{+}(t) \leqq \int_{\mathbb{R}}\left(u_{0}^{\varepsilon}-\hat{u}_{0}^{\varepsilon}\right)^{+} .
$$

By the definition of $(A, B)$ and assumptions (4.14), (6.7), the constants 0 and 1 are evident solutions of (5.1). Hence, the following maximum principle holds: for data $u_{0}^{\varepsilon}$ taking values in $[0,1]$, we have $0 \leqq u^{\varepsilon} \leqq 1$ almost everywhere on $\mathbb{R}_{+} \times \mathbb{R}$. Also the function $\hat{u}(x):=A 1_{\{x<0\}}+B 1_{\{x>0\}}$ is an apparent solution of (5.1). As in the proof of Theorem 6.1, we use uniform estimates on $u^{\varepsilon}$ and compactness arguments to pass to the limit in the Kato inequality written for $u^{\varepsilon}$ and for $\hat{u}^{\varepsilon} \equiv \hat{u}$. What we obtain is the entropy inequality (3.8) with $\left(c^{l}, c^{r}\right)=(A, B)$ and $\mathcal{R}_{\mathcal{G}}=0$ (this is exactly the entropy inequality of BürGER ET AL. [22]). In addition, we see that $u$ is a weak solution of (1.2), (1.7) and it is a Kruzhkov entropy solution in the domains $\{x<0\}$ and $\{x>0\}$. We conclude either using the uniqueness result of [22], or using Proposition 3.17 and the fact that $\mathcal{G}_{(A, B)}$ is definite.

\subsection{Existence for complete germs through the discretization approach}

We now establish the existence of a $\mathcal{G}$-entropy solution for any complete maximal $L^{1} D$ germ $\mathcal{G}$. For the sake of simplicity, let us take $U=\mathbb{R}$. 
Theorem 6.4. Let $\mathcal{G}$ be a complete maximal $L^{1} D$ germ. Assume that the functions $f^{l, r}$ are locally Lipschitz continuous on $\mathbb{R}$. Then for any initial function $u_{0} \in L^{\infty}(\mathbb{R})$ there exists a unique $\mathcal{G}$-entropy solution of problem (1.2), (1.7).

Proof. The proof is a combination of the well-known finite volume method (see EYMARD ET AL. [38]) with a careful treatment of the interface $\{x=0\}$ using the Godunov scheme; the proof of compactness of the family of discrete solutions is based upon the $B V_{\text {loc }}$ estimate of BURGER ET AL. [20,22] and an $L^{\infty}$ bound similar to the one of Proposition 3.20. Note that global (up to the interface) $B V$ estimates are, in general, not available, see [1].

Recall that by the definition of a complete germ, there exists a Riemann solver $\mathcal{R S}^{\mathcal{G}}$ at $\{x=0\}$, fully determined by $\mathcal{G}$, defined for all Riemann data (1.8). We set $m:=\operatorname{ess} \inf _{\mathbb{R}} u_{0}$ (respectively, $M:=\operatorname{ess~sup}_{\mathbb{R}} u_{0}$ ) and denote by $c^{l, r}$ (respectively, by $C^{l, r}$ ) the one-sided traces $\gamma^{l, r} \mathcal{R S}^{\mathcal{G}} m$ (respectively, $\gamma^{l, r} \mathcal{R} \mathcal{S}^{\mathcal{G}} M$ ) of the solution of the Riemann problem at $\{x=0\}$ with the constant datum $u^{ \pm}=m$ (respectively, with the constant datum $\left.u^{ \pm}=M\right)$. Assign

$$
b^{l, r}:=\min \left\{c^{l, r}, m\right\}, \quad B^{l, r}:=\max \left\{C^{l, r}, M\right\} .
$$

We consider an explicit finite volume scheme based on the uniform spatial mesh

$$
\bigcup_{i \in \mathbb{Z}}(i \Delta x,(i+1) \Delta x), \quad \Delta x>0,
$$

and a time step $\Delta t$ satisfying the CFL condition

$$
\lambda:=\frac{\Delta t}{\Delta x} \leqq 1 / 2 L, \quad L:=\max \left\{\max _{z \in\left[b^{l}, B^{l}\right]}\left|\left(f^{l}\right)^{\prime}(z)\right|, \max _{z \in\left[b^{r}, B^{r}\right]}\left|\left(f^{r}\right)^{\prime}(z)\right|\right\} .
$$

A lower bound for $L$ can be obtained from Proposition 3.20.

For $i \neq 0$, we can utilize any consistent monotone flux $g_{i+1 / 2}(\cdot, \cdot)$ (see for example [38]); but at the interface $i=0$ we take $g_{i+1 / 2}(\cdot, \cdot)$ to be the Godunov flux based on the exact $\mathcal{G}$-Riemann solver. Given the numerical flux $g_{i+1 / 2}(\cdot, \cdot)$, the difference scheme is defined as

$u_{i}^{n}=u_{i}^{n-1}-\lambda\left(g_{i+1 / 2}\left(u_{i+1}^{n-1}, u_{i}^{n-1}\right)-g_{i-1 / 2}\left(u_{i}^{n-1}, u_{i-1}^{n-1}\right)\right), \forall n \in \mathbb{N}, \forall i \in \mathbb{Z}$.

where the iteration is initialized by

$$
u_{i}^{0}:=\frac{1}{\Delta x} \int_{i \Delta x}^{(i+1) \Delta x} u_{0}, \quad \forall i \in \mathbb{Z} .
$$

The monotonicity of the numerical flux $g_{i+1 / 2}(\cdot, \cdot)$ and the CFL condition ensure that the scheme can be written as

$$
u_{i}^{n}=H_{i}\left(u_{i-1}^{n-1}, u_{i}^{n-1}, u_{i+1}^{n}\right), \quad \forall n \in \mathbb{N}, \forall i \in \mathbb{Z},
$$

for some functions $H_{i}(\cdot, \cdot, \cdot)$ that are monotone in each of the three arguments. We identify the discrete solution $\left(u_{i}^{n}\right)_{n \in \mathbb{N}, i \in \mathbb{Z}}$ with the piecewise constant function

$$
\mathcal{S}^{h} u_{0}:=\sum_{n \in \mathbb{N}, i \in \mathbb{Z}} u_{i}^{n} 1_{((n-1) \Delta t, n \Delta t) \times(i \Delta x,(i+1) \Delta x))} ;
$$

where $h$ is a collective symbol for the discretization parameters $\Delta x, \Delta t$. 
By construction (exact $\mathcal{G}$-Riemann solver at the interface $\{x=0\}$ ),

the difference scheme preserves all stationary solutions of (1.7)

of the form $c^{l} 1_{\{x<0\}}+c^{r} 1_{\{x>0\}}$ with $\left(c^{l}, c^{r}\right) \in \mathcal{G}$.

First, let us establish the following uniform $L^{\infty}$ bound on $\mathcal{S}^{h} u_{0}$ :

$$
\begin{aligned}
b(x) & :=b^{l} 1_{\{x<0\}}+b^{r} 1_{\{x>0\}} \\
& \leqq\left(\mathcal{S}^{h} u_{0}\right)(t, x) \\
& \leqq B^{l} 1_{\{x<0\}}+B^{r} 1_{\{x>0\}}=: B(x),
\end{aligned}
$$

for almost every $(t, x) \in(0, \infty) \times \mathbb{R}$, where $b^{l, r}, B^{l, r}$ are defined in (6.9). To prove (6.11), we take the Riemann initial datum $b(\cdot)$ and look at the corresponding discrete solution $\left(\mathcal{S}^{h} b\right)(\Delta t, \cdot)$ after the first time step. Denote by $b_{i}^{n}$ the values taken by $\left(\mathcal{S}^{h} b\right)(\Delta t, \cdot)$. Notice that, by the choice of $b^{l, r}$, the exact solution $\mathcal{R} \mathcal{S}^{\mathcal{G}} b$ of the Riemann problem has the one-sided traces $c^{l, r}$ at $\{x=0\}$. Therefore, the Godunov flux $g_{1 / 2}\left(b_{0}^{n}, b_{1}^{n}\right)=g_{1 / 2}\left(b^{l}, b^{r}\right)$ at the interface at time level $n=1$ takes the value $f^{l, r}\left(c^{l, r}\right)$ (the two values being equal). From the definition of the scheme, it is clear that $b_{i}^{1}=b_{i}^{0}$ for all $i \neq 0,1$. Moreover, from the definition of the scheme we have

$$
b_{0}^{1}=b^{l}-\lambda\left(f^{l}\left(c^{l}\right)-f^{l}\left(b^{l}\right)\right), \quad b_{1}^{1}=b^{r}-\lambda\left(f^{r}\left(b^{r}\right)-f^{r}\left(c^{r}\right)\right) .
$$

Because $b^{l} \leqq c^{l}$ by construction, and because the state $b^{l}$ at $x<0$ can be joined to the state $c^{l}$ at $x=0$ - with waves of negative speed for the flux $f^{l}$, it follows that $f^{l}\left(c^{l}\right) \leqq f^{l}\left(b^{l}\right)$. Similarly, we have $f^{r}\left(c^{r}\right) \geqq f^{r}\left(b^{r}\right)$. Combining the above information, we deduce that for all $i \in \mathbb{Z}, b_{i}^{1} \geqq b_{i}^{0}$. Thus we have

$$
\left(\mathcal{S}^{h} b\right)(\Delta t, \cdot) \geqq b(\cdot) .
$$

Hence by induction, using the monotonicity of the scheme, we deduce

$$
\left(\mathcal{S}^{h} b\right)(t, x) \geqq b(x) \text { for almost every }(t, x) \in(0, \infty) \times \mathbb{R} .
$$

Because $u_{0} \geqq b$ almost everywhere on $\mathbb{R}$ and again in view of the monotonicity of the scheme,

$$
\left(\mathcal{S}^{h} u_{0}\right)(t, x) \geqq\left(\mathcal{S}^{h} b\right)(t, x) \geqq b(x) \text { for almost every }(t, x) \in(0, \infty) \times \mathbb{R},
$$

which establishes the desired lower bound in (6.11). The proof of the upper bound is entirely similar.

Now we assume that $u_{0}$ is compactly supported and belongs to $B V(\mathbb{R})$. Then for all $T>0$ and $l>0$, we apply the uniform $B V((0, T) \times(\mathbb{R} \backslash(-l, l)))$ estimate of $[20,22]$ to the family $\left(\mathcal{S}^{h} u_{0}\right)_{h>0}$. To establish this estimate, we first combine the discrete $L^{1}$ contraction property, obtained from the Crandall-Tartar lemma [33], with the $L^{1}$ Lipschitz continuity in time $t$ of the solver $\mathcal{S}^{h}$. The result is an estimate of the time variation of $\mathcal{S}^{h} u_{0}$ on $(0, T) \times \mathbb{R}$ in terms of the variation $\operatorname{Var} u_{0}$ of the initial data $u_{0}$ on $\mathbb{R}$. Then, using the mean value theorem, we pick $l^{h} \in(0, l)$ such that the variation of $\left(\mathcal{S}^{h} u_{0}\right)\left(\cdot, \pm l^{h}\right)$ does not exceed Const $\left(\operatorname{Var} u_{0}\right) / l$. Subsequently, 
we consider $\left.\left(\mathcal{S}^{h} u_{0}\right)\right|_{\mathbb{R} \backslash\left(-l^{h}, l^{h}\right)}$ as originating from the finite volume discretization of two Cauchy-Dirichlet problems. For instance on $(0, T) \times\left(l^{h}, \infty\right)$, both the initial condition $\left.u_{0}\right|_{\left(l^{h}, \infty\right)}$ and the boundary condition $u_{b}(\cdot):=\left(\mathcal{S}^{h} u_{0}\right)\left(\cdot, l^{h}\right)$ have the variation controlled in terms of $\operatorname{Var} u_{0}$ and of $\frac{1}{l}$. It follows that the space-time variation of $\mathcal{S}^{h} u_{0}$ on $(0, T) \times(\mathbb{R} \backslash(-l, l)) \subset(0, T) \times\left(\mathbb{R} \backslash\left(-l^{h}, l^{h}\right)\right)$ is bounded uniformly in $h$; we refer to [20, Lemma 4.2], [22, Lemmas 5.3, 5.4] for the details. Using in addition the $L^{\infty}$ bound, with the help of a diagonal extraction argument, we get convergence as $h \rightarrow 0$ of a (not labelled) sequence $\left(\mathcal{S}^{h} u_{0}\right)_{h}$ to some limit $u$.

Now we justify that the limit $u$ is the unique $\mathcal{G}$-entropy solution of (1.2), (1.7). The above $B V_{\text {loc }}$ estimates (or the standard "weak $B V$ " estimates for monotone finite volume schemes, see [38]) permit us to get an approximate weak formulation and pass to the limit as $h \rightarrow 0$. It follows that $u$ is a weak solution to (1.2), (1.7). In the same way, the technique of [38] allows us to get the Kruzhkov entropy inequalities for $u$ in the domains $\{x<0\}$ and $\{x>0\}$.

According to Definition 3.15 and Proposition 3.17, what remains is to prove the entropy inequality (3.8) with an arbitrary non-negative test function $\xi$ (not necessarily zero on the interface $\{x=0\})$ and with any pair $\left(c^{l}, c^{r}\right) \in \mathcal{G}$. Let us give a proof using (6.10). To this end, notice that a key feature of the scheme, thanks to the use of the Godunov flux of the exact $\mathcal{G}$-Riemann solver at the interface, is that the discrete solutions $u^{h}:=\mathcal{S}^{h} u_{0}$ take the values

$$
\begin{aligned}
& \left(\gamma^{l} u^{h}\right)(\cdot) \equiv \sum_{n \in \mathbb{N}} u_{0}^{n} 1_{((n-1) \Delta t, n \Delta t)}, \\
& \left(\gamma^{r} u^{h}\right)(\cdot) \equiv \sum_{n \in \mathbb{N}} u_{1}^{n} 1_{((n-1) \Delta t, n \Delta t)}
\end{aligned}
$$

at the interface (strong one-sided traces at the interface $\{x=0\}$ ), which satisfy

$$
\left(\left(\gamma^{l} u^{h}\right)(t),\left(\gamma^{r} u^{h}\right)(t)\right) \in \mathcal{G}, \quad \text { for almost every } t>0
$$

Because $\mathcal{G}$ is an $L^{1} D$ germ, it follows that

$$
q^{l}\left(\left(\gamma^{l} u^{h}\right)(t), c^{l}\right) \geqq q^{r}\left(\left(\gamma^{r} u^{h}\right)(t), c^{r}\right), \quad \forall\left(c^{l}, c^{r}\right) \in \mathcal{G},
$$

for almost every $t>0$. Therefore, with the same arguments as in [38], using (6.10) and using in addition the interface dissipation inequality (6.12), we get the required entropy inequalities (3.8) with $\left(c^{l}, c^{r}\right) \in \mathcal{G}$ and zero remainder term.

Summarizing our findings, a $\mathcal{G}$-entropy solution has been constructed for any compactly supported initial function $u_{0}$ in $B V(\mathbb{R})$. It is easy to generalize this result so that it covers all $u_{0} \in L^{\infty}(\mathbb{R})$. Indeed, because of the Lipschitz continuity assumption on $f^{l, r}$, the contraction principle of Theorem 3.19 can be localized (as in the original work of KRUZHKOV [54]); using this contraction principle, by truncation and regularization of $u_{0}$ we construct a strongly compact sequence of approximations $u^{\varepsilon}$. Then we pass to the limit in this sequence of approximations using the formulation of Definition 3.15. This concludes the proof of the theorem. 


\subsection{On convergence of approximate solutions without BV estimates}

In this section, we assume that the existence of a $\mathcal{G}$-entropy solution to the problem (1.2), (1.7) is already known. For existence results, we refer, for example to Sections 6.1, 6.2, 6.3 and to many of the references at the end of the paper. Under this assumption, we have the following general convergence result.

Theorem 6.5. Assume that the fluxes $f^{l, r}$ are merely continuous and the associated maximal $L^{1} D$ germ $\mathcal{G}$ is chosen in such a way that there exists a $\mathcal{G}$-entropy solution to the problem (1.2), (1.7) for all bounded initial functions $u_{0}$.

Suppose that for any $\varepsilon>0$ we are given a map $S^{\varepsilon}: L^{\infty}(\mathbb{R}) \mapsto L^{\infty}\left(\mathbb{R}_{+} \times \mathbb{R}\right)$ with the following properties:

(B1) for each $u_{0} \in L^{\infty}(\mathbb{R})$, the family $\left(S^{\varepsilon} u_{0}\right)_{\varepsilon>0}$ is bounded in $L^{\infty}\left(\mathbb{R}_{+} \times \mathbb{R}\right)$;

(B2) if $\hat{u}_{0}(x)=c^{l} 1_{\{x<0\}}+c^{r} 1_{\{x>0\}}$ with $\left(c^{l}, c^{r}\right) \in \mathcal{G}$,

then $S^{\varepsilon} \hat{u}_{0}$ converges to $\hat{u}_{0}$ almost everywhere on $\mathbb{R}_{+} \times \mathbb{R}$, as $\varepsilon \rightarrow 0$;

(B3) for all $u_{0}, \hat{u}_{0} \in L^{\infty}(\mathbb{R})$ and for all nonnegative $\xi \in \mathcal{D}\left(\mathbb{R}_{+} \times \mathbb{R}\right)$, there exists $r_{1}=r_{1}\left(u_{0}, \hat{u}_{0}, \xi, \varepsilon\right)$, with $r_{1} \rightarrow 0$ as $\varepsilon \rightarrow 0$, such that the following approximate Kato inequality holds:

$$
\begin{gathered}
\int_{\mathbb{R}_{+}} \int_{\mathbb{R}}\left(\left|S^{\varepsilon} u_{0}-S^{\varepsilon} \hat{u}_{0}\right| \xi_{t}+\mathfrak{q}\left(x, S^{\varepsilon} u_{0}, S^{\varepsilon} \hat{u}_{0}\right) \xi_{x}\right) \\
+\int_{\mathbb{R}}\left|u_{0}-\hat{u}_{0}\right| \xi(0, \cdot) \geqq-r_{1}\left(u_{0}, \hat{u}_{0}, \xi, \varepsilon\right) .
\end{gathered}
$$

(B4) for all $u_{0} \in L^{\infty}(\mathbb{R})$ and for all $\xi \in \mathcal{D}\left(\mathbb{R}_{+} \times \mathbb{R}\right)$, there exists $r_{2}=r_{2}\left(u_{0}, \xi, \varepsilon\right)$ with $r_{2} \rightarrow 0$ as $\varepsilon \rightarrow 0$, such that the following approximate weak formulation holds:

$$
\int_{\mathbb{R}_{+}} \int_{\mathbb{R}}\left(S^{\varepsilon} u_{0} \xi_{t}+\mathfrak{f}\left(x, S^{\varepsilon} u_{0}\right) \xi_{x}\right)+\int_{\mathbb{R}} u_{0} \xi(0, \cdot)=r_{2}\left(u_{0}, \xi, \varepsilon\right) .
$$

(B5) for all $u_{0} \in L^{\infty}(\mathbb{R})$, for all nonnegative $\xi \in \mathcal{D}\left(\mathbb{R}_{+} \times(\mathbb{R} \backslash\{x=0\})\right)$, and for all $k \in \mathbb{R}$, there exists $r_{3}=r_{3}\left(u_{0}, k, \xi\right.$, $\left.\varepsilon\right)$, with $r_{3} \rightarrow 0$ as $\varepsilon \rightarrow 0$, such that the following approximate Kruzhkov formulation holds away from the interface $\{x=0\}$ :

$$
\begin{gathered}
\int_{\mathbb{R}_{+}} \int_{\mathbb{R}}\left(\left|S^{\varepsilon} u_{0}-k\right| \xi_{t}+\mathfrak{q}\left(x, S^{\varepsilon} u_{0}, k\right) \xi_{x}\right) \\
\quad+\int_{\mathbb{R}}\left|u_{0}-k\right| \xi(0, \cdot) \geqq-r_{3}\left(u_{0}, k, \xi, \varepsilon\right) .
\end{gathered}
$$

Then, as $\varepsilon \rightarrow 0$, $S^{\varepsilon} u_{0}$ converges almost everywhere on $\mathbb{R}_{+} \times \mathbb{R}$ to the unique $\mathcal{G}$-entropy solution of (1.2), (1.7).

The applications we have in mind are mainly related to numerical approximations. Yet it should be noted that the standard vanishing viscosity approximations of Sections 6.1 fulfill assumptions (B1)-(B5).

In this context, the property (B2) is somewhat delicate: it clearly holds for $\left(c^{l}, c^{r}\right) \in \mathcal{G}_{V V}^{s}$, then it can be extended to $\left(c^{l}, c^{r}\right) \in \mathcal{G}_{V V}$, using the fact that $\mathcal{G}_{V V}$ 
is the closure of $\mathcal{G}_{V V}^{s}$ (see Proposition 5.1) and using the continuity with respect to the initial function $u_{0}$ of the vanishing viscosity solver $S^{\varepsilon}$.

Thanks to Theorem 6.5, from the existence result of Theorem 6.4 we deduce

Corollary 6.6. If the vanishing viscosity germ $\mathcal{G}_{V V}$ is complete, then the following assumption can be dropped from Theorem 6.1:

$$
f^{l} \text { and } f^{r} \text { are not affine on any interval } I \subset[0,1] .
$$

The germ $\mathcal{G}_{V V}$ is known to be complete in many situations (see [34-37,42,43]). We expect that the vanishing viscosity germ $G_{V V}$ is complete whenever $f^{l, r}$ are compactly supported, and also in the case there exist two sequences of elementary $G_{V V}$-entropy solutions $\left(b_{k}(\cdot)\right)_{k>1}$ and $\left(B_{k}(\cdot)\right)_{k>1}$ such that as $k \rightarrow \infty, b_{k} \rightarrow-\infty$ and $B_{k} \rightarrow \infty$.

In general, assumption (B2) appears to be too restrictive. Certainly it holds for numerical schemes using either the exact Riemann solver on the interface $\{x=0\}$, or the associated Godunov flux, but it may become difficult to justify in other situations. For instance, we cannot simply combine Theorems 6.4 and 6.5 in order to drop assumption (6.13) from the statement of Theorem 6.3; this is so because only the connection solution $A 1_{\{x<0\}}+B 1_{\{x>0\}}$ and the two constant states 0 and 1 are preserved by the adapted viscosity approximation (6.6). However, the investigation of the approximate solutions for Riemann initial data of the form $u_{0}=c^{l} 1_{\{x<0\}}+c^{r} 1_{\{x>0\}}$, with $\left(c^{l}, c^{r}\right) \in \mathcal{G}_{(A, B)} \backslash\{(A, B),(0,0),(1,1)\}$, would require subtler arguments.

Note that whenever the strong compactness property is available, the schemes that preserve only some definite germ $\mathcal{G}_{0}$ strictly smaller than $\mathcal{G}$ do converge, thanks to the formulation of Proposition 3.17 (see [9,22,51] for a few particular cases).

Proof of Theorem 6.5. First, in view of (B1), we have nonlinear weak- $\star$ compactness of the sequence $\left(S^{\varepsilon} u_{0}\right)_{\varepsilon>0}$. Combining it with the strong convergence of $S^{\varepsilon} \hat{u}_{0}$, with $\hat{u}^{0}$ being any stationary solution from (B2), we pass to the limit as $\varepsilon \rightarrow 0$ (up to extraction of a subsequence) in the formulations (B3), (B4), and (B5). Then the nonlinear weak- $\star$ limit $\mu$ of (a subsequence of) $S^{\varepsilon} u_{0}$ is a $\mathcal{G}$-entropy process solution, according to Remark 3.25. Applying Theorem 3.28, we conclude that $\mu$ is identified with the unique $\mathcal{G}$-entropy solution $u$ of (1.2), (1.7) and $u$ is therefore the unique accumulation point of $\left(S^{\varepsilon} u_{0}\right)_{\varepsilon>0}$ in $L_{\text {loc }}^{1}\left(\mathbb{R}_{+} \times \mathbb{R}\right)$. This concludes the proof.

Acknowledgements. This paper was written as part of the research program on Nonlinear Partial Differential Equations at the Centre for Advanced Study at the Norwegian Academy of Science and Letters in Oslo, which took place during the academic year 2008-2009. The authors thank S. Mishra, D. Mitrovič, E. Panov, N. Seguin, and J. Towers for interesting discussions.

Open Access This article is distributed under the terms of the Creative Commons Attribution Noncommercial License which permits any noncommercial use, distribution, and reproduction in any medium, provided the original author(s) and source are credited. 


\section{References}

1. Adimurthi, Ghoshal, S.S., Dutta, R., Veerappa Gowda, G.D.: Existence and nonexistence of TV bounds for scalar conservation laws with discontinuous flux. Comm. Pure Appl. Math. 64(1), 84-115 (2011)

2. Adimurthi, Veerappa Gowda, G.D.: Conservation laws with discontinuous flux. J. Math. Kyoto University 43(1), 27-70 (2003)

3. Adimurthi, Jaffré, J., Veerappa Gowda, G.D.: Godunov-type methods for conservation laws with a flux function discontinuous in space. SIAM J. Numer. Anal. 42(1), 179-208 (2004)

4. Adimurthi, Mishra, S., Veerappa Gowda, G.D.: Optimal entropy solutions for conservation laws with discontinuous flux-functions. J. Hyperbolic Differ. Equ. 2(4), 783837 (2005)

5. Alt, H.W., Luckhaus, S.: Quasilinear elliptic-parabolic differential equations. Mat. Z. 183, 311-341 (1983)

6. Ammar, K., Wittbold, P.: Existence of renormalized solutions of degenerate ellipticparabolic problems. Proc. Roy. Soc. Edinburgh Sect. A 133(3), 477-496 (2003)

7. Ammar, K., Wittbold, P., Carrillo, J.: Scalar conservation laws with general boundary condition and continuous flux function. J. Differ. Equ. 228(1), 111-139 (2006)

8. Andreianov, B., Bénilan, P., KruZhkov, S.N.: $L^{1}$ theory of scalar conservation law with continuous flux function. J. Funct. Anal. 171(1), 15-33 (2000)

9. Andreianov, B., Goatin, P., Seguin, N.: Finite volume schemes for locally constrained conservation laws. Numer. Math. 115(4), 609-645 (2010)

10. Andreianov, B., Karlsen, K.H., Risebro, N.H.: A theory of $L^{1}$-dissipative solvers for scalar conservation laws with discontinuous flux. II (in preparation)

11. Andreianov, B., Karlsen, K.H., Risebro, N.H.: On vanishing viscosity approximation of conservation laws with discontinuous flux. Netw. Heterog. Media 5(3), 617-633 (2010)

12. Andreianov, B., Sbini, K.: Scalar conservation laws with nonlinear boundary conditions. C. R. Acad. Sci. Paris, Ser. I 345, 431-434 (2007)

13. Audusse, E., Perthame, B.: Uniqueness for scalar conservation laws with discontinuous flux via adapted entropies. Proc. Roy. Soc. Edinburgh A 135(2), 253-265 (2005)

14. Bachmann, F., Vovelle, J.: Existence and uniqueness of entropy solution of scalar conservation laws with a flux function involving discontinuous coefficients. Comm. Partial Differ. Equ. 31, 371-395 (2006)

15. Baiti, P., Jenssen, H.K.: Well-posedness for a class of $2 \times 2$ conservation laws with $L^{\infty}$ data. J. Differ. Equ. 140(1), 161-185 (1997)

16. Bardos, C., LeRoux, A.-Y., Nédélec, J.-C.: First order quasilinear equations with boundary conditions. Comm. Partial Differ. Equ. 4(9), 1017-1034 (1979)

17. BÉnilan, P.: Equations d'évolution dans un espace de Banach quelconques et applications. Thèse d'état, 1972

18. Bénilan, P., Carrillo, J., Wittbold, P.: Renormalized entropy solutions of scalar conservation laws. Ann. Scuola Norm. Sup. Pisa Cl. Sci. (4) 29(2), 313-327 (2000)

19. BÉnilan, P., Kruzhrov, S.N.: Conservation laws with continuous flux functions. NoDEA Nonlinear Differ. Equ. Appl. 3(4), 395-419 (1996)

20. Bürger, R., García, A., Karlsen, K.H., Towers, J.D.: Difference schemes, entropy solutions, and speedup impulse for an inhomogeneous kinematic traffic flow model. Netw. Heterog. Media 3, 1-41 (2008)

21. Bürger, R., Karlsen, K.H., Mishra, S., Towers, J.D.: On conservation laws with discontinuous flux. Trends in Applications of Mathematics to Mechanics (Eds. Wang Y. and Hutter K.) Shaker Verlag, Aachen, 75-84, 2005

22. Bürger, R., Karlsen, K.H., Towers, J.: An Engquist-Osher type scheme for conservation laws with discontinuous flux adapted to flux connections. SIAM J. Numer. Anal. 47, 1684-1712 (2009) 
23. CANCÈs, C.: Asymptotic behavior of two-phase flows in heterogeneous porous media for capillarity depending only on space. I. Convergence to the optimal entropy solution. SIAM J. Math. Anal. 42(2), 946-971 (2010)

24. CANCÈs, C.: Asymptotic behavior of two-phase flows in heterogeneous porous media for capillarity depending only on space. II. Nonclassical shocks to model oil-trapping. SIAM J. Math. Anal. 42(2), 972-995 (2010)

25. CANCÈs, C.: On the effects of discontinuous capillarities for immiscible two-phase flows in porous media made of several rock-types. Netw. Heterog. Media 5(3), 635-647 (2010)

26. Carrillo, J.: Entropy solutions for nonlinear degenerate problems. Arch. Ration. Mech. Anal. 147(4), 269-361 (1999)

27. Chechin, G.A., Goritsky, A.Yu.: S. N. Kruzhkov's lectures on first-order quasilinear PDEs. (Eds. Emmrich E. and Wittbold P.) Anal. Numer. Asp. Partial Differ. Equ., de Gruyter, 2009

28. Chen, G.-Q., Even, N., Klingenberg, C.: Hyperbolic conservation laws with discontinuous fluxes and hydrodynamic limit for particle systems. J. Differ. Equ. 245(11), 3095-3126 (2008)

29. Chen, G.-Q., Frid, H.: Divergence-measure fields and hyperbolic conservationlaws. Arch. Ration. Mech. Anal. 147, 89-118 (1999)

30. Chen, G.-Q., Rascle, M.: Initial layers and uniqueness of weak entropy solutions to hyperbolic conservation laws. Arch. Ration. Mech. Anal. 153, 205-220 (2000)

31. Colombo, R.M., Goatin, P.: A well posed conservation law with a variable unilateral constraint. J. Differ. Equ. 234(2), 654-675 (2007)

32. Crandall, M.G.: The semigroup approach to first-order quasilinear equations in several space variables. Israel J. Math. 12, 108-132 (1972)

33. Crandall, M.G., Tartar, L.: Some relations between nonexpansive and order preserving mappings. Proc. Am. Math. Soc. 78(3), 385-390 (1980)

34. DienL, S.: On scalar conservation laws with point source and discontinuous flux function. SIAM J. Math. Anal. 26(6), 1425-1451 (1995)

35. DienL, S.: Scalar conservation laws with discontinuous flux function. I. The viscous profile condition. II. On the stability of the viscous profiles. Commun. Math. Phys. 176(1), 23-44 and 45-71 (1996)

36. DieHL, S.: A conservation law with point source and discontinuous flux function modelling continuous sedimentation. SIAM J. Appl. Math. 56(2), 388-419 (1996)

37. DieHL, S.: A uniqueness condition for non-linear convection-diffusion equations with discontinuous coefficients. J. Hyperbolic Differ. Equ. 6(1), 127-159 (2009)

38. Eymard, R., Gallouët, T., Herbin, R.: Finite volume methods. Handbook of numerical analysis, Vol. VII, Handb. Numer. Anal., VII. North-Holland, Amsterdam, 7131020, 2000

39. Gallouët, T., Hubert, F.: On the convergence of the parabolic approximation of a conservation law in several space dimensions. Chin. Ann. Math. Ser. B 20(1), 7-10 (1999)

40. Garavello, M., Natalini, R., Piccoli, B., Terracina, A.: Conservation laws with discontinuous flux. Netw. Heterog. Media 2, 159-179 (2007)

41. Gelfand, I.M.: Some problems in the theory of quasilinear equations. (Russian) Uspekhi Mat. Nauk 14(2), 87-158, 1959; English tr. in Am. Math. Soc. Transl. Ser. 29(2), 295-381 (1963)

42. Gimse, T., Risebro, N.H.: Riemann problems with a discontinuous flux function. Proceedings of Third International Conference on Hyperbolic Problems, Vol. I, II Uppsala, 1990, 488-502, Studentlitteratur, Lund, 1991

43. Gimse, T., Risebro, N.H.: Solution of the Cauchy problem for a conservation law with a discontinuous flux function. SIAM J. Math. Anal. 23(3), 635-648 (1992)

44. Holden, H., Risebro, N.H.: Front tracking for hyperbolic conservation laws. In: Applied Mathematical Sciences, vol. 152. Springer, New York, 2002 
45. Hopf, E.: The partial differential equation $u_{t}+u u_{x}=\mu u_{x x}$. Comm. Pure Appl. Math. 3(3), 201-230 (1950)

46. JimeneZ, J.: Some scalar conservation laws with discontinuous flux. Int. J. Evol. Equ. 2(3), 297-315 (2007)

47. JimENEZ, J., LÉvi, L.: Entropy formulations for a class of scalar conservations laws with space-discontinuous flux functions in a bounded domain. J. Engrg. Math. 60(3-4), 319-335 (2008)

48. KaAsschieter, E.F.: Solving the Buckley-Leverett equation with gravity in a heterogeneous porous medium. Comput. Geosci. 3, 23-48 (1999)

49. Karlsen, K.H., Towers, J.D.: Convergence of the Lax-Friedrichs scheme and stability for conservation laws with a discontinuous space-time dependent flux. Chin. Ann. Math. Ser. B. 25(3), 287-318 (2004)

50. Karlsen, K.H., Risebro, N.H., Towers, J.D.: On a nonlinear degenerate parabolic transport-diffusion equation with a discontinuous coefficient. Electron J. Differ. Equ., pages No. 93, 23 pp. (electronic), (2002)

51. KarlsEN, K.H., Risebro, N.H., Towers, J.D.: Upwind difference approximations for degenerate parabolic convection-diffusion equations with a discontinuous coefficient. IMA J. Numer. Anal. 22, 623-664 (2002)

52. KARLSEN, K.H., Risebro, N.H., TowERs, J.D.: $L^{1}$ stability for entropy solutions of nonlinear degenerate parabolic convection-diffusion equations with discontinuous coefficients. Skr. K. Nor. Vidensk. Selsk. 3, 1-49 (2003)

53. Klingenberg, C., Risebro, N.H.: Convex conservation laws with discontinuous coefficients: Existence, uniqueness and asymptotic behavior. Comm. Partial Differ. Equ. 20(11-12), 1959-1990 (1995)

54. KruzhKov, S.N.: First order quasi-linear equations in several independent variables. Math. USSR Sbornik 10(2), 217-243 (1970)

55. KruzhKov, S.N., Hildebrand, F.: The Cauchy problem for first order quasilinear equations in the case when the domain of dependence on the initial data is infinite (Russian). Vestnik Moskov. Univ. Ser. I Mat. Meh. 29,93-100 (1974)

56. KruzhKov, S.N., PANOv, E.Yu.: First-order quasilinear conservation laws with infinite initial data dependence area (Russian). Dokl. Akad. Nauk URSS 314(1), 79-84, 1990; English tr. in Sov. Math. Dokl. 42(2), 316-321 (1991)

57. Kwon, Y.-S., VAsseur, A.: Strong traces for solutions to scalar conservation laws with general flux. Arch. Ration. Mech. Anal. 185(3), 495-513 (2007)

58. LeFLOCH, P.G.: Hyperbolic systems of conservation laws. Lectures in Mathematics ETH Zürich. Birkhäuser Verlag, Basel, 2002. The theory of classical and nonclassical shock waves

59. Lions, J.-L.: Quelques méthodes de résolution des problèmes aux limites non linéaires. (French) Dunod, Paris, 1969

60. Lions, P.-L., Perthame, B., Tadmor, E.: A kinetic formulation of multidimensional scalar conservation laws and related equations. J. Am. Math. Soc. 7(1), 169-191 (1994)

61. Málek, J., NeČAs, J., RoKyta, M., RužičKa, M.: Weak and Measure-valued Solutions to Evolutionary PDEs. Chapman \& Hall, London, 1996

62. Maliki, M., Touré, H.: Uniqueness of entropy solutions for nonlinear degenerate parabolic problems. J. Evol. Equ. 3(4), 603-622 (2003)

63. Mishra, S.: Analysis and Numerical Approximation of Conservation Laws with Discontinuous Coefficients. PhD thesis, Indian Institute of Science, Bangalore, India, 2005

64. Mitrovič, D.: Existence and stability of a multidimensional scalar conservation law with discontinuous flux. Networks Het. Media 5(1), 163-188 (2010)

65. OLENIK, O.A.: Uniqueness and stability of the generalized solution of the Cauchy problem for a quasi-linear equation. Amer. Math. Soc Transl. Ser. 2 33, 285-290 (1963)

66. Ostrov, D.N.: Solutions of Hamilton-Jacobi equations and scalar conservation laws with discontinuous space-time dependence. J. Differ. Equ. 182(1), 51-77 (2002)

67. Отто, F.: Initial-boundary value problem for a scalar conservation law. C. R. Acad. Sci. Paris Sér. I Math. 322(8), 729-734 (1996) 
68. Отто, F.: $L^{1}$-contraction and uniqueness for quasilinear elliptic-parabolic equations. J. Differ. Equ. 131(1), 20-38 (1996)

69. Panov, E.Yu.: Strong measure-valued solutions of the Cauchy problem for a first-order quasilinear equation with a bounded measure-valued initial function. Moscow Univ. Math. Bull. 48(1), 18-21 (1993)

70. Panov, E.Yu.: On sequences of measure valued solutions for a first order quasilinear equation (Russian). Mat. Sb. 185(2), 87-106 1994; Engl. tr. in Russian Acad. Sci. Sb. Math. 81(1), 211-227 (1995)

71. PAnov, E.Yu.: Existence of strong traces for generalized solutions of multidimensional scalar conservation laws. J. Hyperbolic Differ. Equ. 2(4), 885-908 (2005)

72. Panov, E.Yu.: Existence of strong traces for quasi-solutions of multidimensional conservation laws. J. Hyperbolic Differ. Equ. 4(4), 729-770 (2007)

73. PAnov, E.Yu.: Existence and strong pre-compactness properties for entropy solutions of a first-order quasilinear equation with discontinuous flux. Arch. Ration. Mech. Anal. 195(2), 643-673 (2009)

74. Panov, E.Yu.: On existence and uniqueness of entropy solutions to the Cauchy problem for a conservation law with discontinuous flux. J. Hyperbolic Differ. Equ. 6(3), 525-548 (2009)

75. Perthame, B.: Kinetic formulation of conservation laws, volume 21 of Oxford Lecture Series in Mathematics and its Applications. Oxford University Press, Oxford, 2002

76. Quinn (Keyfitz), B.: Solutions with shocks, an example of an $L^{1}$-contractive semigroup. Comm. Pure Appl. Math. 24(1), 125-132 (1971)

77. Seguin, N., Vovelle, J.: Analysis and approximation of a scalar conservation law with a flux function with discontinuous coefficients. Math. Models Methods Appl. Sci. 13(2), 221-257 (2003)

78. SzEPESSY, A.: Measure-valued solution of scalar conservation laws with boundary conditions. Arch. Ration. Mech. Anal. 107(2), 182-193 (1989)

79. Temple, B.: Global solution of the Cauchy problem for a class of $2 \times 2$ nonstrictly hyperbolic conservation laws. Adv. Appl. Math. 3(3), 335-375 (1982)

80. Towers, J.D.: Convergence of a difference scheme for conservation laws with a discontinuous flux. SIAM J. Numer. Anal. 38(2), 681-698 (2000)

81. Towers, J.D.: A difference scheme for conservation laws with a discontinuous flux: the nonconvex case. SIAM J. Numer. Anal. 39(4), 1197-1218 (2001)

82. Vallet, G.: Dirichlet problem for a nonlinear conservation law. Rev. Math. Comput. 13(1), 231-250 (2000)

83. VASSEUR, A.: Strong traces of multidimensional scalar conservation laws. Arch. Ration. Mech. Anal. 160(3), 181-193 (2001)

84. Vol'Pert, A.I.: The spaces BV and quasi-linear equations. Math. USSR Sbornik 2(2), 225-267 (1967)

Laboratoire de Mathématiques CNRS UMR 6623,

Université de Franche-Comté,

16 route de Gray 25030

Besancon,

France.

e-mail: boris.andreianov@univ-fcomte.fr

and 
Centre of Mathematics for Applications,

University of Oslo,

P.O. Box 1053, Blindern,

0316 Oslo,

Norway.

e-mail: kennethk@math.uio.no

URL: http://folk.uio.no/kennethk/

and

Centre of Mathematics for Applications,

University of Oslo,

P.O. Box 1053, Blindern, 0316 Oslo,

Norway.

e-mail: nilshr@math.uio.no

URL: http://folk.uio.no/nilshr/

(Received April 23, 2010 / Accepted September 25, 2010)

Published online January 12, 2011 - (C) The Authors(s) (2011)

This article is published with open access at Springerlink.com 